\title{
Energy Pattern Aided Simultaneous Wireless Information and Power Transfer
}

\author{
Rong Zhang, Member, IEEE, Lie-Liang Yang, Senior Member, IEEE, and Lajos Hanzo, Fellow, IEEE
}

\begin{abstract}
In echoing Varshney's seminal concept of jointly transmitting information and energy, we propose the concept of an energy pattern aided Simultaneous Wireless Information and Power Transfer (SWIPT) system, where in addition to power transfer, information is conveyed both by the specific Receive Antenna (RA) indices to which the power is delivered as well as by the particular intensity of the power assigned to that particular RA pattern. By embedding information into energy patterns rather than imposing it by modulating classic radio waveforms, our proposed solution is capable of operating both in an integrated receiver mode and in a power-split mode, while relying on a low-complexity two-stage non-coherent detection algorithm. Both our analysis and simulations show that our energy pattern aided SWIPT system exhibits a beneficial immunity to any potential performance degradation imposed by power-conversion. Moreover, the achievable rate versus power conversion trade-off bounds are characterized, demonstrating that our proposed energy pattern aided SWIPT system leads to a beneficial wireless information and power transfer convergence.
\end{abstract}

Index Terms-Simultaneous wireless information and power transfer, multiple input multiple output, transmitter pre-coding, pre-coded spatial modulation.

\section{INTRODUCTION}

1) Motivation: In statistical physics, there is a fundamentally under-explored and much-debated topic concerning information to energy conversion. The history of this subject has evolved from the early thought experiment of 1867 known as "Maxwell's Demon" [1] to the landmark philosophy referred to as "Landauer's principle" [2] in the mid-20th century as well as to the development of reversible electronics [3] and to the information heat engine [4] of our modern era. These seminal contributions suggested from a fundamental perspective the joint treatment of information and energy in future engineering systems [5]. Although it is a challenge to design practical systems benefiting from information to energy conversion, the explicit concept of transporting both information and energy simultaneously was also raised by the authors of [6], [7]. This topic was then further extended to the practical realms of wireless communications [8], since it is desirable to ensure

Manuscript received April 1, 2014; revised September 15, 2014; accepted December 16, 2014. The financial support of the EPSRC under the India-U.K. Advanced Technology Centre (IU-ATC), that of the EU under the Concerto project as well as that of the European Research Council's (ERC) Advanced Fellow Grant is gratefully acknowledged.

The authors are with the Southampton Wireless, School of ECS, University of Southampton, Southampton SO17 1BJ, U.K. (e-mail: rz@ecs.soton.ac.uk; 1ly@ecs.soton.ac.uk; lh@ecs.soton.ac.uk; http://www-mobile.ecs.soton.ac.uk).

Color versions of one or more of the figures in this paper are available online at http://ieeexplore.ieee.org.

Digital Object Identifier 10.1109/JSAC.2015.2391551 that a mobile device is entirely free from being tethered, as prophesied by Tesla a century ago [9]. Evolving from the above intriguing theoretical ideas, it is the maturing Wireless Power Transfer (WPT) and wireless communications fields that make the Simultaneous Wireless Information and Power Transfer (SWIPT) an important emerging research topic.

In the field of WPT, we refer the reader to a brief historical portrayal in [10]. Broadly speaking, WPT can be carried out in two basic ways, namely based on either electromagnetic induction in the form of inductive coupling and resonant coupling relying on coils [11] or with the aid of electromagnetic radiation using microwave frequencies by relying on so-called rectennas [12]. Early microwave power transmission was conceived in high-power applications in the Mega/Kilo-Watt-range in the context of the microwave-powered helicopter [13] and in the National Aeronautics and Space Administration's (NASA) feasibility study of the space-solar program [14]. Owing to the development of electronic devices in the late 20th century, research has been focused on the design of compact and efficient rectennas conceived for low-power applications in the milli/micro-Watt-range [15], [16], where the start-up company Powercast ${ }^{1}$ has reported that micro-Watt-scale power was transmitted over a distance of a few meters at the transmission power of $23 \mathrm{dBm}$ at a frequency around $900 \mathrm{MHz}$. Another state-of-the-art implementation was reported very recently in [17], which relied on a microwave meta-material that converts microwave energy into electric power at a conversion efficiency comparable to that of modern solar panels.

Owing to the fact that the electromagnetic radiation is restricted by both health and safety regulations, it remains an open challenge at the current state-of-the-art to power a mobile phone, typically requiring around hundreds of milliWatt power. Nonetheless, this technology is already appealing for employment in low-power sensors. Consequently, it becomes especially promising for the near-future wireless systems, where a compelling solution was proposed by the Mobile and wireless communications Enablers for the Twenty-twenty Information Society (METIS) project [18] with the goal of connecting billions of low-power devices globally. In this scenario, known as Machine Type Communications (MTC) [19] or the "Internet Of Things" (IOT) [20], SWIPT is expected to become a pervasive enabler to create the IoT, which is immortal from an energy replenishment perspective, "to facilitate and cheapen the transmission of intelligence" [9].

2) Background: The current research of SWIPT is still in its infancy, hence there is a paucity of literature. The pioneering

\footnotetext{
${ }^{1}$ See websites: http://www.powercastco.com/.
} 
work of [6] revealed that a non-trivial trade-off exists between energy and capacity for typical channel models, such as the Binary Symmetric Channel (BSC) and the Additive White Gaussian Noise (AWGN) channel, indicating that maximizing the information rate is to a degree coupled with maximizing the energy transfer. When a wideband fading channel is considered, the authors of [8] found that a fundamental trade-off exists between energy and capacity, where the classic water-filling power allocation across the entire frequency band maximizes the attainable information rate, while energy transfer is maximized by transmitting at a single frequency using the total power available, thus reducing the information rate. Similar conclusions were also drawn concerning the fundamental tradeoff for narrowband Multiple Input Multiple Output (MIMO) channels [21], where water-filling based power allocation spanning all eigenvalues of the channel-matrix maximizes the information rate. By contrast, the energy transfer is maximized by concentrating all available power in the specific direction corresponding to the maximum eigenvalue. Further research addressed various other classic channel models, such as the interference channels [22], multiple access channels [23], unicast/multicast channels [24] and secrecy channels [25].

Alongside those theoretical results, practical receiver architectures facilitating SWIPT may be operated in two different modes [21], namely either on a time-division basis or on a power-split basis. To be more specific, in the time-division mode, the receiver alternatively and opportunistically acts as an information detector and power converter [26]. On the other hand, in the power-split mode, the required portion of the received power is used for powering the receiver, while the remaining received power is used for retrieving information [27]. Finally, a hybrid receiver architecture integrating both information and power transfer was proposed in [28]. The respective trade-offs between energy and capacity were characterized for all three receiver architectures of [21], [26]-[28].

In addition to the above one-way SWIPT focusing on receiver architectures, the scope of SWIPT may also be further expanded. For example, the authors of [29], [30] investigated the scenario, where the transmitter conveys power to the receiver, which is then converted to Direct Current (DC) power and reused for the destination's information transmission in the reverse direction. This mode of operation is similar to that of the passive Radio-frequency IDentification (RFID) devices [31] and hence it may be referred to as being two-way half-duplex. More radically, the authors of [32] investigated a two-way fullduplex operational mode, where a pair of nodes interactively communicates and exchanges power. This is also reminiscent of the so-called energy cooperation [33]. Finally, the following set of treatises considered a range of energy-transfer-aided systems, such as multi-carrier systems [34], [35], relay-assisted systems [36] and beamforming-aided systems [37], [38].

3) Novelty: A close inspection of the existing literature reveals that most of the current designs are centred on the energy versus capacity trade-offs, on the receiver architectures and on the attainable system performance under power transfer constraints. It would be however equally beneficial to aim for the full "convergence" of information and power transfer as the ultimate objective in the spirit of Varshney's seminal concept [7], which proposed that energy and information transfer should be innately inter-linked.

Naturally, information is carried by attaching itself to a physical medium, such as radio waves or particles. In optical communications, information is delivered by photons having information-dependent intensities, which may be detected by a photon-counting process [39], [40]. Given the nature of the process, the system is capable of providing a heating/ illumination/propulsion function. Analogously, with the aid of the MIMO concept, we propose a novel technique, where information is carried not by the classic radio waveforms but by energy patterns. More explicitly, our pattern energy embeds information into the specific pattern of the power delivered, which may assume

- a position-based energy pattern, which is reminiscent of the Pulse Position Modulation (PPM) concept, but being invoked in the Spatial Domain (SD).

- an intensity-based energy pattern, which is similar to Pulse Amplitude Modulation (PAM), but exclusively relying on positive values.

Novelty: in this paper, we introduce the energy pattern aided SWIPT concept, where the information is conveyed not only by the Receive Antenna (RA) index of the power delivered but also by the specific intensity of the delivered power. The achievable rate of our technique is immune to any power conversion, thus leading to a beneficial wireless information and power transfer convergence.

The rest of our paper is organised as follows. In Section II, we introduce the underlying concept as well as the transceiver architecture of our energy pattern aided SWIPT system followed by the portrayal of its detection algorithm and its achievable rate analysis. Our simulation results are provided in Section III, while we conclude in Section IV.

\section{SYSTEM DESCRIPTION}

Let us now introduce the underlying concept of energy pattern aided SWIPT as well as its receiver modes, detection algorithm and achievable rate analysis.

\section{A. SWIPT Transmitter}

Consider a MIMO system equipped with $N_{t}$ Transmit Antennas (TA) and $N_{r}$ RA, where we assume $N_{t} \geq N_{r}$. The proposed energy pattern aided SWIPT transmitter relies on the availability of Channel State Information at the Transmitter (CSIT) for supporting Transmitter Pre-Coding (TPC). ${ }^{2}$

1) RA Index-Based Energy Pattern: Our RA index-based energy pattern is generated with the aid of our recently proposed Generalised Pre-coding aided Spatial Modulation (GPSM) scheme [41], [42]. Explicitly, a total of $N_{a}<N_{r}$ RAs are activated, where the particular pattern of the $N_{a}$ RAs activated conveys information in form of so-called SD

\footnotetext{
${ }^{2}$ This scenario has been widely considered in the SWIPT literature [21], [22], [29], [37], [38], since it is desirable to shift most/all signal processing demands from the less powerful receiver that may be passive or semi-passive to the more powerful transmitter that may have access to the mains power.
} 


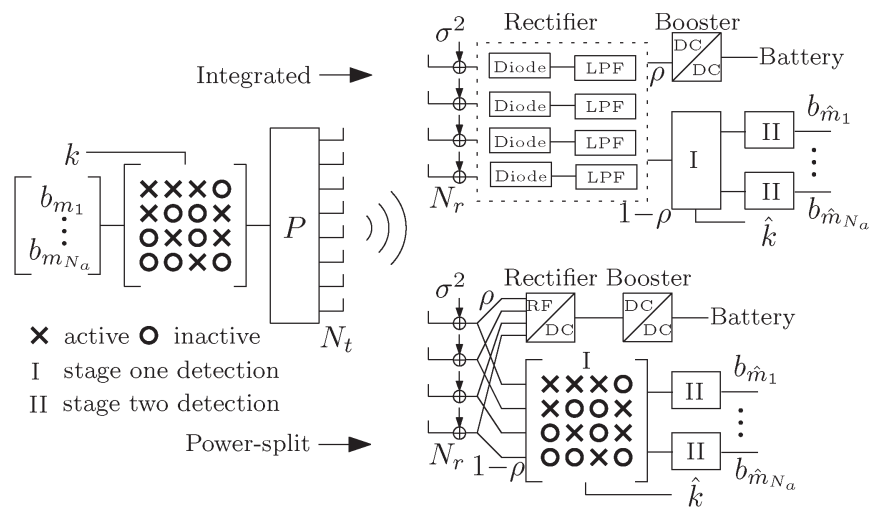

Fig. 1. Transceiver architecture of energy pattern aided SWIPT relying either on an integrated receiver (top) or a power-split receiver (bottom). For example, activating $N_{a}=2$ RAs out of $N_{r}=4$ RAs results in a total of $\left|\mathcal{C}_{t}\right|=6$ legitimate activation patterns, i.e., the patterns of $\mathfrak{C}_{t}=$ $\{[1,2],[1,3],[1,4],[2,3],[2,4],[3,4]\}$. This configuration delivers $k_{p}=2$ bits of information, because $\left\lfloor\log _{2}(|6|)\right\rfloor=2$. Upon selecting for example $\mathcal{C}=$ $\left\{\mathfrak{C}_{t}(1), \mathfrak{C}_{t}(2), \mathfrak{C}_{t}(3), \mathfrak{C}_{t}(4)\right\}$, we have the following mapping between the SD symbol and the activation patterns $k=1 \mapsto \mathfrak{C}(1)=[1,2], k=2 \mapsto \mathfrak{C}(2)=[1,3]$, $k=3 \mapsto \mathcal{C}(5)=[1,4]$, and $k=4 \mapsto \mathcal{C}(6)=[2,3]$.

symbols. Hence, the number of bits conveyed by a SD symbol becomes $k_{p}=\left\lfloor\log _{2}\left(\left|\mathcal{C}_{t}\right|\right)\right\rfloor$, where the set $\mathcal{C}_{t}$ contains all the combinations associated with choosing $N_{a}$ activated RAs out of $N_{r}$ RAs. $^{3}$ To assist our further discussions, let us define the set of selected activation patterns representing a RA index-set as $\mathcal{C} \subset \mathcal{C}_{t}$. We also let $\mathcal{C}(k)$ and $\mathcal{C}(k, i)$ denote the $k$ th $\mathrm{RA}$ activation pattern and the $i$ th activated RA in the $k$ th activation pattern, respectively. An example is given in the caption of Fig. 1.

Let $\boldsymbol{s}_{p}^{k} \in \mathbb{R}_{++}^{N_{r} \times 1}$ be a super-symbol indicating that the RA pattern represented by the index $k$ is activated and $N_{a}$ unit energy pulses $\boldsymbol{b}_{p}=\mathbf{1}^{N_{a} \times 1}$ are delivered. In other words, we have the relationship of

$$
\boldsymbol{s}_{p}^{k}=\Omega_{k} \boldsymbol{b}_{p}
$$

where $\Omega_{k}=\boldsymbol{I}[:, \mathrm{C}(k)]$ is constituted by the specifically selected columns determined by $\mathcal{C}(k)$ of an identity matrix of $\boldsymbol{I}_{N_{r}}$. Following the TPC operation of $\boldsymbol{P} \in \mathbb{C}^{N_{t} \times N_{r}}$, the resultant transmit signal $\boldsymbol{x} \in \mathbb{C}^{N_{t} \times 1}$ may be written as

$$
\boldsymbol{x}=\sqrt{\beta / N_{a}} \boldsymbol{P} \boldsymbol{s}_{p}^{k}
$$

As a natural requirement, the TPC matrix has to ensure that no energy leaks into the RA-elements of the unintended RA patterns. Hence, the classic linear Channel Inversion (CI)-based TPC [43], [44] may be used, which is formulated as

$$
\boldsymbol{P}=\boldsymbol{H}^{H}\left(\boldsymbol{H} \boldsymbol{H}^{H}\right)^{-1}
$$

where $\boldsymbol{H} \in \mathbb{C}^{N_{r} \times N_{t}}$ represents the MIMO channel involved. Furthermore, to avoid any power fluctuation during the preprocessing, we introduce the scaling factor of $\beta$ designed for maintaining the power-constraint of $\mathbb{E}\left[\|x\|^{2}\right]=1$.

\footnotetext{
${ }^{3}$ Note that having a fractional number of bits conveyed by a SD symbol is also possible, but we restrict this number to be an integer in this paper for the ease of implementation and analysis.
}

2) Intensity-Based Energy Pattern: Our intensity-based energy pattern is generated by replacing the above $N_{a}$ unit energy pulses $\boldsymbol{b}_{p}$ with information-bearing energy pulses representing our symbols

$$
\boldsymbol{b}_{m}=\left[b_{m_{1}}, \ldots, b_{m_{N_{a}}}\right]^{T} \in \mathbb{R}_{++}^{N_{a} \times 1}
$$

where we have $b_{m_{i}} \in \mathcal{A}, i \in\left[1, N_{a}\right]$ with its alphabet denoted by $\mathcal{A}$. By dropping the index, let us focus on $b_{m}$, where a total of $k_{s}=\log _{2}(M)$ bits are mapped to one of $M=|\mathcal{A}|$ equi-probable energy pulse based symbols and $\mathbb{E}\left[b_{m}^{2}\right]=1$.

We commence by designing the energy pulse based symbol alphabet relying on Equi-spaced Amplitudes (EA), which are $\mathcal{A}_{E A}=\{1 / d, 2 / d, \ldots, M / d\}$, where we have the energy pulse weights

$$
d=\sqrt{\frac{1+2^{2}+\cdots+M^{2}}{M}}=\sqrt{\frac{(M+1)(2 M+1)}{6}} .
$$

Alternatively, we can design the energy pulse based symbol alphabet relying on Equi-spaced Powers (EP), which are $\mathcal{A}_{E P}=\{1 / d, \sqrt{2} / d, \ldots, \sqrt{M} / d\}$, where we have the energy pulse weights

$$
d=\sqrt{(1+2+\cdots+M) / M}=\sqrt{(M+1) / 2} .
$$

Finally, the optimum design of the alphabet using optimally spaced symbols is left for our future work.

3) Energy Pattern: When combining RA index-based energy pattern and intensity-based energy pattern, (2) becomes

$$
\boldsymbol{x}=\sqrt{\beta / N_{a}} \boldsymbol{P} \boldsymbol{s}_{m}^{k}=\sqrt{\beta / N_{a}} \boldsymbol{P} \boldsymbol{\Omega}_{k} \boldsymbol{b}_{m} .
$$

As a result, the total number of bits transmitted by our energy pattern is $k_{e}=k_{p}+N_{a} k_{s}$. Furthermore, by letting $v_{i}=\mathcal{C}(k, i)$, the scaling factor $\beta$ becomes

$$
\begin{aligned}
\beta & =\frac{N_{a}}{\sum_{i=1}^{N_{a}} \boldsymbol{R}_{v_{i}, v_{i}}^{-1}+\mathbb{E}_{\mathcal{C}}(k)\left[\chi \sum_{i=1}^{N_{a}} \sum_{j=1, j \neq i}^{N_{a}} \boldsymbol{R}_{v_{i}, v_{j}}^{-1}\right]}, \\
& \approx \frac{N_{r}}{\operatorname{Tr}\left[\boldsymbol{R}^{-1}\right]+\chi \sum_{i=1}^{N_{r}} \sum_{j=1, j \neq i}^{N_{r}} \boldsymbol{R}_{i, j}^{-1}}
\end{aligned}
$$

where we have $\boldsymbol{R}=\boldsymbol{H} \boldsymbol{H}^{H}$ and $\chi$ denotes the correlation of the energy pulse based symbols in the intensity-based energy pattern, which is given as

$$
\chi \in\left\{\chi_{E A}, \chi_{E P}\right\}=\left\{\frac{3(M+1)}{4 M+2}, \frac{2\left(\sum_{\mathrm{int}=1}^{M} \sqrt{\mathrm{int}}\right)^{2}}{(M+1)}\right\} .
$$

Finally, the Base Band (BB) signal $x$ is then up-converted to generate the Radio Frequency (RF) signal $x^{R F}$ at a carrier frequency of $f_{c}$ and conveyed through $N_{t}$ TAs.

\section{B. SWIPT Receiver}

The RF signal observed at the $N_{r}$ RAs may be written as

$$
\boldsymbol{r}^{R F}=\boldsymbol{H} \boldsymbol{x}^{R F}+\boldsymbol{w}^{R F}=\sqrt{2} \mathcal{R}\left\{(\boldsymbol{H} \boldsymbol{x}+\boldsymbol{w}) e^{j 2 \pi f_{c} t}\right\}
$$

where $\mathcal{R}\{\cdot\}$ denotes the real operator and $\boldsymbol{w}^{R F}$ is the RF receiver's noise. The equivalent $\mathrm{BB}$ representation of the 
noise $\boldsymbol{w} \in \mathbb{C}^{N_{r} \times 1}$ may be modelled by a circularly symmetric complex-valued Gaussian noise vector with each entry having a zero mean and a variance of $\sigma^{2}$, i.e., we have $\mathbb{E}\left[\|\boldsymbol{w}\|^{2}\right]=\sigma^{2} N_{r}$. Furthermore,

$$
r_{i}^{R F}=\sqrt{2} \mathcal{R}\left\{\left(\boldsymbol{h}_{i} \boldsymbol{x}+w_{i}\right) e^{j 2 \pi f_{c} t}\right\}
$$

represents the RF signal observed at the $i$ th RA and $r_{i}=\boldsymbol{h}_{i} \boldsymbol{x}+w_{i}$ denotes the equivalent BB representation of $r_{i}^{R F}$, where $\boldsymbol{h}_{i}$ is the ith row of $\boldsymbol{H}$.

1) Integrated Model: The integrated receiver concept of Fig. 1 was introduced in [28], where the received RF power is firstly converted to DC power by the rectifiers consisting of a diode and a passive Low-Pass Filter (LPF). Considering (11) and following the analytical models advocated in [15], the current $c_{i}$ output by the non-linear diode for the $i$ th RA is

$$
c_{i}=I_{s}\left(e^{\alpha r_{i}^{R F}}-1\right)=a_{1} r_{i}^{R F}+a_{2}\left(r_{i}^{R F}\right)^{2}+\cdots,
$$

where the coefficients obey $a_{n}=I_{s} \alpha^{n} / n !, n=1,2, \cdots$ with $I_{s}$ being the saturation current and $\alpha$ being the reciprocal of the thermal voltage. Following [28], we reformulate (11)as $r_{i}^{R F}=\sqrt{2}\left|r_{i}\right| \mathcal{R}\left\{e^{2 \pi f_{c} t+\theta}\right\}$ with $\theta=\angle r_{i}$ and insert it into (12), leading to

$$
\begin{aligned}
c_{i} & \approx \sqrt{2} a_{1}\left|r_{i}\right| \mathcal{R}\left\{e^{2 \pi f_{c} t+\theta}\right\}+2 a_{2}\left|r_{i}\right|^{2}\left(\mathcal{R}\left\{e^{2 \pi f_{c} t+\theta}\right\}\right)^{2} \\
& =a_{2}\left|r_{i}\right|^{2}+\sqrt{2} a_{1}\left|r_{i}\right| \mathcal{R}\left\{e^{2 \pi f_{c} t+\theta}\right\}+a_{2}\left|r_{i}\right|^{2} \mathcal{R}\left\{e^{2 \pi 2 f_{c} t+\theta}\right\} .
\end{aligned}
$$

The first approximation of (13) exploits the fact that the impact of higher powers of $r_{i}^{R F}$ is negligible, thus only the first two terms of (12) are considered. Subsequently, the LPF at the output of the diode removes the harmonic components at $f_{c}$ and $2 f_{c}$ of (13), thus we have the DC current of

$$
c_{i}^{0}=\operatorname{LPF}\left(c_{i}\right)=a_{2}\left|r_{i}\right|^{2} .
$$

Note that $a_{2}=I_{s} \alpha^{2} / 2$ is expressed in unit of Amp/Watt and since it is a constant determined by the diode, we assume it to be of unity. ${ }^{4}$ By normalising with respect to $a_{2}$, the DC power harvested is proportional to $\left|r_{i}\right|^{2}$.

After converting the power from RF to DC, the power-splitter of Fig. 1 is employed, resulting into a power transfer path and an information transfer path. For the power transfer path, a portion $\rho$ of the DC power is converted, which is followed by a (multistage) DC to DC booster and energy storage. As a result, the average power transferred by the transmitter and harvested from all the $N_{r}$ RAs is represented as

$$
Q \propto Q=\mathbb{E}\left[\xi \rho \sum_{i=1}^{N_{r}}\left|\tilde{r}_{i}\right|^{2}\right]
$$

where $\tilde{r}_{i}$ denotes the noiseless part of $r_{i}$, representing the power purposely transferred by the transmitter. For the time being, the design of adaptive RA specific power-split ratios $\rho_{i}, i \in\left[1, N_{r}\right]$

\footnotetext{
${ }^{4}$ In [28], the additional noise term of the rectifiers is included and a pulse energy modulation was designed when rectifiers noise is dominant than the antenna noise. However, in this paper, we assume that the RF noise is dominant, where $\boldsymbol{w}$ accounts for both the thermal noise and for the interference.
}

is set aside for our future investigations and we simply set the $\mathrm{RF}$ to DC conversion efficiency to $\xi=0.5$, noting that, it has a non-linear relationship with respect to the incident RF power.

On the other hand, the remaining $1-\rho$ portion of the DC power enters the information transfer path, which may be described for the $i$ th RA as

$$
\mathcal{D}_{i}=(1-\rho)\left|\boldsymbol{h}_{i} \boldsymbol{x}+w_{i}\right|^{2} .
$$

Then the $N_{r}$ detection variables $\left\{\mathcal{D}_{1}, \mathcal{D}_{2}, \cdots, \mathcal{D}_{N_{r}}\right\}$ are used for retrieving the information carried by the energy pattern.

2) Power-Split Mode: The power-split receiver concept of Fig. 1 was introduced in [21], where we have a power transfer path and an information transfer path at the front-end. Explicitly, the received RF signal will be first subject to power conversion at each RA using a power-split ratio of $\rho$. By replacing $r_{i}^{R F}$ in (12) by its power-split version of $\sqrt{\rho} r_{i}^{R F}$ and following the same methodology, the average power transferred by the transmitter and harvested from all the $N_{r}$ RAs is represented the same as for the integrated mode in (15).

After RF to DC power conversion at each RA at a ratio of $\rho$, the remaining RF signal observed at the $i$ th RA may be written as

$$
y_{i}^{R F}=\sqrt{2(1-\rho)} \mathcal{R}\left\{\left(\boldsymbol{h}_{i} \boldsymbol{x}+w_{i}\right) e^{j 2 \pi f_{c} t}\right\}
$$

To retrieve the information carried by the energy pattern, the following $N_{r}$ detection metrics are required $\left\{\left|y_{1}^{R F}\right|^{2},\left|y_{2}^{R F}\right|^{2}, \ldots,\left|y_{N_{r}}^{R F}\right|^{2}\right\}$, where

$$
\left|y_{i}^{R F}\right|^{2}=\left|y_{i}\right|^{2}=(1-\rho)\left|\boldsymbol{h}_{i} \boldsymbol{x}+w_{i}\right|^{2} .
$$

Note that (18) is the same as (16), suggesting that both receiver modes share the same energy detection-like algorithm [45] and hence in the following we will work with (18). We will also show later that $\rho$ has to be set asymptotically close to unity for maximizing the power conversion of (15), since its value does not affect the information transfer based on (18) or (16).

\section{Detection Algorithm}

Following the above discussions, let us focus on the lowcomplexity two-stage detection algorithm by firstly determining the RA indices (SD symbol) of the delivered power and then detecting the intensity (energy pulse based symbol) of the power delivered at that position, while allowing us to defer the optimal joint detection to Section II-E3 owing to its high complexity.

1) Stage-One Detection: The SD symbol may be determined by simply sorting the received power accumulated by each legitimate RA pattern, which is mathematically represented as

$$
\hat{k}=\arg \max _{\ell \in[1,|\mathcal{E}|]}\left\{\sum_{i=1}^{N_{a}}\left|y_{\mathcal{C}(\ell, i)}\right|^{2}\right\} .
$$

Thus, correct hard detection is declared, when we have $\hat{k}=k$. We now formulate the proposition that the power-split ratio $\rho$ does not affect the stage-one detection. 
Proposition II.1: The RA pattern detection of (19) is immune to the power conversion operation of (15) for $\rho<1$.

Proof: As long as we have $c>0$, it can be observed that the decision based on the following metric is equivalent to (19), namely

$$
\hat{k}=\arg \max _{\ell \in[1,|\mathcal{C}|]}\left\{c \sum_{i=1}^{N_{a}}\left|y_{\mathcal{C}(\ell, i)}\right|^{2}\right\} .
$$

Letting $c=1 /(1-\rho)$, we have $\left|y_{\mathcal{C}(\ell, i)}\right|^{2} /(1-\rho)=\left|r_{\mathcal{C}(\ell, i)}\right|^{2}$, which implies that $\rho$ does not affect the decision.

2) Stage-Two Detection: As a subsequent stage after the RA pattern determination, the hard detection of energy pattern intensity invokes the Maximum-Likelihood (ML) criterion, as formulated in Lemma II.1:

Lemma II.1: (Proof in Appendix A) The ML criterion conceived for retrieving the information encapsulated in the intensity-based energy pattern of the $\hat{v}_{i}=\mathcal{C}(\hat{k}, i)$ th RA is

$$
\hat{m}_{i}=\arg \max _{n_{i} \in[1,|\mathcal{A}|]} \mathcal{L}=\arg \max _{n_{i} \in[1,|\mathcal{A}|]} f_{\chi_{2}^{2}}\left(g ; \lambda b_{n_{i}}^{2}\right)_{g=A}
$$

where $\mathcal{L}$ represents the likelihood of the energy pulse based symbol $b_{n_{i}} \in \mathcal{A}$ that results into the observation of $A=\left|r_{\hat{v}_{i}}\right|^{2} / \sigma_{0}^{2}$, where $\sigma_{0}^{2}=\sigma^{2} / 2$. Furthermore, $f_{\chi_{2}^{2}}\left(g ; \lambda b_{n_{i}}^{2}\right)$ represents the Probability Distribution Function (PDF) of the non-central chisquare distribution having two degrees of freedom and a noncentrality of $\lambda b_{n_{i}}^{2}$, where $\lambda=\beta / N_{a} \sigma_{0}^{2}$.

Thus, correct hard detection is declared, when we have $\hat{m}_{i}=$ $m_{i}, i \in\left[1, N_{a}\right]$. We now have the following proposition that the power-split ratio $\rho$ does not affect the stage-two detection.

Proposition II.2: The intensity-based energy pulse symbol detection of (21) is immune to the power conversion operation of (15) for $\rho<1$.

Proof: This is explicit in (21), since the likelihood $\mathcal{L}$ is not a function of the factor $(1-\rho)$. This can be also seen from Appendix A, because the factor $(1-\rho)$ in (35) has been eliminated by normalisation.

\section{Achievable Rate}

Both Proposition II.1 and II.2 suggest that $\rho$ has to be set asymptotically close to unity for maximizing the power conversion of (15), since its value does not affect the information transfer by the operations of (19) and (21), i.e. neither for the power-split receiver nor for the integrated receiver. This implies that for $\rho<1$, the advocated energy pattern aided SWIPT system attains the same information transfer performance. Hence, we proceed by quantifying the achievable rate of the advocated energy patten aided SWIPT system for $\rho<1$.

1) Expression of Achievable Rate: In the previous contributions, Shannon's Continuous-input Continuousoutput Memoryless Channel (CCMC) capacity is quantified under the implicit assumption that the input signal obeys a Gaussian distribution. By contrast, our energy pattern aided SWIPT system is special in the sense that the SD symbols used for RA index-based information transfer convey integer values. Similarly, the energy pulse symbols used for intensity-based information transfer are also in discrete format. Thus the employment of the so-called Discrete-input Continuousoutput Memoryless Channel (DCMC) capacity becomes more appropriate. Since the notion of DCMC capacity implicitly relies on the idealised assumptions of having both an optimal detector and capacity-approaching channel codes, we focus our attention on practical receivers, namely the two-stage detections, where the achievable rate obeying Discrete-input discrete-output Memoryless Channel (DMC) is quantified instead of the DCMC capacity. ${ }^{5}$

More explicitly, we quantify the achievable rate, when employing the two-stage detection algorithm described in Section II-C, where the Mutual Information per Bit (MIB) $I(z ; \hat{z})$ of our energy patten aided SWIPT system measured between the input bits $z \in\{0,1\}$ and the corresponding detected output bits $\hat{z} \in\{0,1\}$ obeys the classic DMC model and it is hence given by:

$$
I(z ; \hat{z})=H(z)-H(z \mid \hat{z})
$$

where $H(z)=-\sum_{z} P_{z} \log _{2} P_{z}$ represents the entropy of the input bits $z$ and $P_{z}$ is the Probability Mass Function (PMF) of $z$. It is noted furthermore that we have $H(z)=1$, when we adopt the common assumption of equal-probability bits, i.e., $P_{z=0}=$ $P_{z=1}=1 / 2$. On the other hand, the conditional entropy $H(z \mid \hat{z})$ represents the average uncertainty concerning $z$ after observing $\hat{z}$, which is given by:

$$
\begin{aligned}
H(z \mid \hat{z}) & =\sum_{\hat{z}} P_{\hat{z}}\left[\sum_{z} P_{z \mid \hat{z}} \log _{2} P_{z \mid \hat{z}}\right] \\
& =-e_{\times} \log e_{\times}-\left(1-e_{\times}\right) \log \left(1-e_{\times}\right)
\end{aligned}
$$

where $e_{\times}$is the crossover probability. By substituting (23) into (22) and exploiting $H(z)=1$ we have:

$$
\begin{aligned}
I(z ; \hat{z}) & =1+e_{\times} \log e_{\times}+\left(1-e_{\times}\right) \log \left(1-e_{\times}\right) \\
& =1+e_{b}^{e} \log e_{b}^{e}+\left(1-e_{b}^{e}\right) \log \left(1-e_{b}^{e}\right)
\end{aligned}
$$

where $e_{b}^{e}$ is the effective Bit Error Ratio (BER) of our energy patten aided SWIPT system representing the transition probability of the DMC. As a result, the achievable rate becomes

$$
R=k_{e} I(z ; \hat{z}) .
$$

2) Expression of Error Probability: Let $e_{b}^{p}\left(e_{s}^{p}\right)$ and $e_{b}^{s}\left(e_{s}^{s}\right)$ represent the BER (Symbol Error Ratio (SER)) of the SD symbol for RA index-based information transfer and of the energy pulse symbol for intensity-based information transfer, respectively. The effective BER may be written as

$$
e_{b}^{e}=\left(k_{p} e_{b}^{p}+N_{a} k_{s} e_{b}^{s}\right) / k_{e} \approx\left(\delta_{k_{p}} e_{s}^{p}+N_{a} e_{s}^{s}\right) / k_{e}
$$

\footnotetext{
${ }^{5}$ Importantly, our achievable rate is calculated for the two-stage detection advocated and this is why it is not referred to as the "capacity". Thus, the achievable rate would naturally become different, when different receivers were to be employed. For example, when soft detection combined with channel coding is employed, the error probability would become lower at low SNRs, leading to a higher achievable rate. When optimal detection combined with idealised capacity-achieving channel coding is employed, the achievable rate would be approaching the DCMC capacity.
} 
where the second half of (26) follows from the approximation of $e_{s}^{s} \approx e_{b}^{s} k_{s}$ as a result of Gray mapping and from the relation $e_{s}^{p} \approx e_{b}^{p} k_{p} / \delta_{k_{p}}$ with the correction factor $\delta_{k_{p}}$ given in Lemma II.2. Thus we find that for the sake of calculating (24) and hence (25), we need the expression of $e_{s}^{p}$ and $e_{s}^{s}$, as formulated in Lemma II.3 and Lemma II.4, respectively.

Lemma II.2: (Proof in Appendix B) The generic expression of the correction factor $\delta_{k_{p}}$ for $k_{p}$ bits is given by:

$$
\delta_{k_{p}}=\delta_{k_{p}-1}+\frac{2^{k_{p}-1}-\delta_{k_{p}-1}}{2^{k_{p}}-1}
$$

where given $\delta_{0}=0$, we can recursively determine $\delta_{k_{p}}$.

Lemma II.3: (Proof in Appendix C) The analytical SER $e_{s}^{p}$ of the SD symbol for RA index-based information transfer may be upper bounded by $e_{s, u b}^{p}$ as:

$$
e_{s}^{p}<1-\mathbb{E}_{\boldsymbol{b}_{m}}\left[\int_{0}^{\infty} \bar{e}_{s, l b}^{p}\left(\lambda, \boldsymbol{b}_{m}\right) f_{\lambda}(\lambda) d \lambda\right]=e_{s, u b}^{p}
$$

where we have $\lambda=\beta / N_{a} \sigma_{0}^{2}$ and the lower bound of the probability of correct detection of the SD symbol when the energy pattern based symbol $\boldsymbol{b}_{m}$ was transmitted becomes

$$
\bar{e}_{s, l b}^{p}\left(\lambda, \boldsymbol{b}_{m}\right)=\prod_{i=1}^{N_{a}} \int_{0}^{\infty}\left[F_{\chi_{2}^{2}}(g)\right]^{N_{r}-N_{a}} f_{\chi_{2}^{2}}\left(g ; \lambda b_{m_{i}}^{2}\right) d g
$$

where $F_{\chi_{2}^{2}}(g)$ represents the Cumulative Distribution Function (CDF) of a chi-square distribution having two degrees of freedom, while $f_{\chi_{2}^{2}}\left(g ; \lambda b_{m_{i}}^{2}\right)$ represents the PDF of a non-central chi-square distribution having two degrees of freedom and noncentrality given by $\lambda b_{m_{i}}^{2}$ with the PDF of $f_{\lambda}(\lambda)$.

Lemm II.4: (Proof in Appendix D) The analytical SER $e_{s}^{s}$ of the energy pulse based symbols for intensity-based information transfer may be upper bounded by $e_{s, u b}^{s}$ as:

$$
e_{s}^{s}<\left(1-e_{s, u b}^{p}\right) \tilde{e}_{s, u b}^{s}+e_{s, u b}^{p} \frac{\sum_{\ell \neq k} N_{c} \tilde{e}_{s, u b}^{s}+N_{d} e_{s}^{o}}{N_{a}\left(2^{k_{p}}-1\right)}=e_{s, u b}^{s}
$$

where $N_{c}$ and $N_{d}=\left(N_{a}-N_{c}\right)$ represent the number of common and different RA between $\mathrm{C}(\ell)$ and $\mathrm{C}(k)$, respectively. Furthermore, $e_{s}^{o}=(M-1) / M$ and $\tilde{e}_{s, u b}^{s}$ denotes the SER upper bound of the energy pulse based symbols in the absence of SD symbol errors, which is given by (31), where $\lambda=\beta / N_{a} \sigma_{0}^{2}, b_{m} \in \mathcal{A}$ and $t_{m}$ as seen in Appendix D.

$$
\begin{aligned}
\tilde{e}_{s, u b}^{s}=\frac{1}{M} \int_{0}^{\infty}[ & 1-F_{\chi_{2}^{2}}\left(g ; \lambda b_{1}^{2}\right)_{g=t_{1}} \\
& +F_{\chi_{2}^{2}}\left(g ; \lambda b_{M}^{2}\right)_{g=t_{M-1}} \\
& +\sum_{m=2}^{M-1} F_{\chi_{2}^{2}}\left(g ; \lambda b_{m}^{2}\right)_{g=t_{m-1}} \\
& \left.+1-F_{\chi_{2}^{2}}\left(g ; \lambda b_{m}^{2}\right)_{g=t_{m}}\right] f_{\lambda}(\lambda) d \lambda .
\end{aligned}
$$

Finally, we get the upper bound of the effective BER by substituting the (28) and (30) into (26). This is then used to get the lower bound of (24) and of (25), respectively, where we use the simplified notation of $I$ and $R$, instead of $I^{l b}$ and $R^{l b}$.

\section{E. Further Discussions}

Let us finally address some further issues concerning the energy pattern aided SWIPT system advocated.

1) Derivatives: Let us for a moment consider only the RA index-based energy pattern, where unit-energy pulses $\boldsymbol{b}_{p}$ are used in conjunction with $\chi=1$ in (8). Hence, using the stageone detection of (19) is sufficient. Another potential systemconfiguration is when only the intensity-based energy pattern detection of (21) is invoked in conjunction with $N_{a}=N_{r}$.

When the information is embedded in the TA indices, rather than in the RA indices, we arrive at the so-called Spatial Modulation (SM) concept [46]. However, SM may not be used in a SWIPT system relying on an integrated receiver, because Zero-Forcing (ZF) would be required at the receiver for determining the TA indices. This BB operation cannot be performed on the energy metrics of (16) harvested at the output of rectifiers and this holds also true for ML-based SM receivers. Similarly, the ingenious Sub-carrier Index Modulation (SIM) of [47] achieved by embedding information in sub-carrier index patterns may also be unsuitable for SWIPT systems relying on an integrated receiver because of the BB Fast Fourier Transform (FFT) operation.

2) Alternatives: Without using the CI-based TPC, we may design an alternative energy pattern aided SWIPT system by employing a Maximum Eigenvalue (ME) aided TPC. In the ME-TPC aided arrangement, only a single information stream is precoded using the right-singular vector $\boldsymbol{\mu}_{r}^{*}$ corresponding to the maximum eigenvalue $\lambda_{e}^{*}$ of the MIMO channel $\boldsymbol{H}$. However, the challenge is that the left-singular vector $\boldsymbol{\mu}_{l}^{*}$ corresponding to $\lambda_{e}^{*}$ of the MIMO channel $\boldsymbol{H}$ used for post-processing at the receiver is also required for intensity-based information transfer. Although this post-processing is typically applied at $\mathrm{BB}$, based on the solutions developed in [48], [49], it may also be realised at the RF front-end [50], [51]. ${ }^{6}$ Thus, in the ME-TPC aided arrangement, first post-processing is applied at $\mathrm{RF}$ and as a result, the associated power conversion becomes capable of harvesting $\mathcal{Q} \propto Q=\mathbb{E}\left[\xi \rho \lambda_{e}^{*} b_{m}^{2}\right]$ amount of power. By assigning all the available power to the direction corresponding to the maximum eigenvalue, the amount of transferred power is thus maximised at the cost of a reduced information rate, since only a single information stream is supported as detailed in [21]. As for the information transfer, the metric $\left|\lambda_{e}^{*} b_{m}+\boldsymbol{\mu}_{l}^{*} \boldsymbol{w}\right|^{2}$ is formed, which is then maximized as seen in (21).

As an important practical aspect, let us now consider the channel knowledge requirement of our energy pattern aided SWIPT system. For our CI-TPC aided arrangement, the stageone detection of (19) is entirely non-coherent, hence no channel knowledge is required at the receiver. By contrast, the stagetwo detection of (21) needs the estimates of $\beta$, which may be approximated according to [52]. Hence, we may assume a Time Division Duplex (TDD) operation for the acquisition of CSIT, where the receiver sends its uplink pilot sequences to the transmitter, which are used for estimating the uplink channel.

\footnotetext{
${ }^{6}$ The beamforming solutions based on SVD plus waterfilling and its variants are excluded here, since they rely on post-multiplication by the conjugate of the left unitary matrix of the channel's SVD, which can only be realised in the baseband.
} 
The resultant uplink channel information is then calibrated and reused for the downlink SWIPT, which assumes reciprocity between the uplink and downlink channels in the TDD mode. On the other hand, in the ME-TPC aided arrangement, both the transmitter and the receiver requires the availability of channel knowledge, thus it is more complex and less practical than the CI-TPC aided arrangement.

3) Complications: We now discuss the optimal joint detection of both the intensity-based energy pattern and of the SD symbol $k$. By defining $\mathcal{D}=\left\{\left|y_{1}\right|^{2}, \cdots,\left|y_{N_{r}}\right|^{2}\right\}$, the ML criterion based optimal joint detection is formulated as

$$
\left[\hat{m}_{1}, \ldots, \hat{m}_{N_{a}}, \hat{k}\right]=\underset{\boldsymbol{s}_{n}^{\ell} \in \mathcal{B}}{\arg \max } p\left(\mathcal{D} \mid \boldsymbol{s}_{n}^{\ell}\right)
$$

where $\mathcal{B}=\mathcal{C} \times \mathcal{A}^{N_{a}}$ is the joint search space of the supersymbol $\boldsymbol{s}_{n}^{\ell}$, which may be explicitly expanded as $\mathcal{B}=\{\ell \in$ $\left.[1,|\mathcal{C}|], n_{i} \in[1,|\mathcal{A}|], i \in\left[1, N_{a}\right]\right\}$. More explicitly, the likelihood function $\mathcal{L}_{n}^{\ell}=p\left(\mathcal{D} \mid \boldsymbol{s}_{n}^{\ell}\right)$ is given as

$$
\mathcal{L}_{n}^{\ell}=\prod_{i=1}^{N_{a}} f_{\chi_{2}^{2}}\left(\frac{\left|r_{\mathcal{C}(\ell, i)}\right|^{2}}{\sigma_{0}^{2}} ; \lambda_{n_{i}}^{2}\right) \prod_{j=1}^{N_{a}^{\prime}} f_{\chi_{2}^{2}}\left(\frac{\left|r_{\overline{\mathcal{C}}(\ell, j)}\right|^{2}}{\sigma_{0}^{2}}\right)
$$

where we have $N_{a}^{\prime}=N_{r}-N_{a}, \lambda=\beta / N_{a} \sigma_{0}^{2}$ and $\sigma_{0}^{2}=\sigma^{2} / 2$. Since the expansion of (33) becomes trivial with the aid of our proofs of Lemma II.1 and II.3 in Appendix A and C, we omit it for the sake of space economy.

It is thus clear that the two-stage detection algorithm advocated in Section II-C constitutes a low-complexity alternative to the joint detection of (32). More explicitly, the two-stage detection represents a sequential low-complexity implementation of the joint detection, where the stage-one detection finds the RA pattern with respect to arbitrary $\boldsymbol{b}_{n}$, while the stagetwo detection finds the most likely $\boldsymbol{b}_{n}$ given the estimate of the SD symbol $\hat{k}$ provided by the stage-one detection. It is also clear that the joint detection has a complexity on the order of $|\mathcal{C}| \times|\mathcal{A}|^{N_{a}}$, while our two-stage detection has a complexity on the order of $|\mathcal{C}|+N_{a}|\mathcal{A}|$.

Proposition II.3: The stage-one SD symbol detection of (19) obeys the ML criterion.

Proof: In the stage-one detection of Section II-C1, given an arbitrary $\boldsymbol{b}_{n} \in \mathcal{A}^{N_{a}}$, the optimal ML criterion for determining the $\mathrm{SD}$ symbol $\hat{k}$ is formulated as

$$
\begin{aligned}
& \hat{k}=\arg \max _{\ell \in[1,|\mathcal{E}|]} p\left(\mathcal{D} \mid \Omega_{\ell}, \boldsymbol{b}_{n}\right)=\arg \max _{\ell \in[1,|\mathcal{E}|]} \mathcal{L}_{n}^{\ell} \\
& \stackrel{a}{a} \arg \max _{\ell \in[1,|\mathcal{E}|]} \prod_{i=1}^{N_{a}} I_{0}\left(\sqrt{\frac{\lambda b_{n_{i}}^{2}}{(1-\rho) \sigma_{0}^{2}}\left|y_{\mathcal{C}(\ell, i)}\right|^{2}}\right) \\
& \stackrel{b}{\propto \arg } \max _{\ell \in[1,|\mathcal{E}|]}\left\{\prod_{i=1}^{N_{a}}\left|y_{\mathcal{C}(\ell, i)}\right|^{2}\right\} \stackrel{c}{\propto} \underset{\arg }{\max }\left\{\prod_{\ell \in[1,|\mathcal{E}|]}^{N_{a}} e_{i=1}^{\left|y_{\mathcal{E}(\ell, i)}\right|^{2}}\right\} \\
& \propto \arg \max _{\ell \in[1,|\mathcal{C}|]}\left\{\sum_{i=1}^{N_{a}}\left|y_{\mathcal{C}(\ell, i)}\right|^{2}\right\}
\end{aligned}
$$

where in (34-a) we omitted the irrelevant terms after expanding (33). Furthermore, in (34-b) and (34-c), we exploited the fact that the modified zeroth-order first kind Bessel function $I_{0}(\cdot)$ and the exponential function are both monotonically increasing positive convex function.

\section{Performance Evaluation}

Let us now characterize the performance of our energy pattern aided SWIPT system advocated.

\section{A. Configuration}

In our simulations, the transmit power is set to $P_{t}=30 \mathrm{dBm}$ or 1 watt and the path-loss model obeys $P_{r}=P_{t} A_{t} A_{r}\left(d_{0} \lambda_{c}\right)^{-\alpha_{p l}}$, where $d_{0}$ is the distance between TA and RA array, $\lambda_{c}$ denotes the wavelength corresponding to a carrier frequency of 5.8 $\mathrm{GHz}$, the path-loss exponent is set to $\alpha_{p l}=4$ and the aperture of the TA and RA is represented by $A_{t}$ and $A_{r}$, respectively with the per-antenna aperture being set to $1 \mathrm{~cm}^{2}$. Each entry of the channel-matrix $\boldsymbol{H}$ undergoes frequency-flat Rician fading and it is uncorrelated between the different super-symbol instants, but remains constant within the duration of a supersymbol. When modelling the Line Of Sight (LOS) path of the Rician channel, we employ the far-field uniform linear antenna array at both the transmitter and receiver [53], with their responses represented as $\left[1, e^{-j \pi / 2}, \cdots, e^{-j \pi\left(N_{t}-1\right) / 2}\right]$ and as $\left[1, e^{-j \pi / 2}, \cdots, e^{-j \pi\left(N_{r}-1\right) / 2}\right]$, respectively. We assume having perfect channel information at both the receiver as well as at the transmitter and set aside the effects of CSIT-errors for our future investigations [37], [41]. Finally, the RF antenna induced noise $w$ accounts for both the thermal noise as well as for the interference and it is thus set to $\sigma^{2}=-50 \mathrm{dBm}$. This assumption is valid as for the non-licence band at $5.8 \mathrm{GHz}$ considered, interference could be pervasive. Also, the RF to DC conversion efficiency is set to $\xi=0.5$.

\section{B. Preparatory Results}

1) BER Performance: Fig. 2 shows both the analytical and simulated performance of a $\left\{N_{t}, N_{r}\right\}=\{8,4\}$ energy pattern aided SWIPT system, characterizing its effective BER (left) for $M$-ary intensity-based energy pattern using the equi-spaced amplitudes $\mathcal{A}_{E A}$ and experiencing Rayleigh fading with $\rho<1$. In addition, we also characterize the BER relying on the RA index-based energy pattern in isolation (right), namely the BER as a result of pure stage-one detection. It is clear that our analytical results form a tight upper bound of the simulated results in both subplots of Fig. 2. As expected, when the RA's distance from the TAs increases, the BER performance degrades in both subfigures of Fig. 2. Furthermore, as seen in the left subplot of Fig. 2, the higher the ratio of $N_{a} / N_{t}$, the worse the BER performance becomes. Similarly, observe in the right subplot of Fig. 2 that a higher number of activated RA leads to a degraded BER performance, since the receiver becomes more prone to decision errors.

2) Information Transfer: Fig. 3 shows the achievable rate of a $\left\{N_{t}, N_{r}\right\}=\{8,4\}$ energy pattern aided SWIPT system employing various $M$-ary intensity-based energy pattern using the equi-spaced amplitudes $\mathcal{A}_{E A}$ and experiencing Rayleigh fading

\footnotetext{
${ }^{7}$ Practically, the RF to DC conversion efficiency has a non-linear relationship with respect to the incident RF power, which is closely related to the particular design of the power conversion circuits. Setting it to 0.5 constitutes a realistic choice, since the true value reported at http://www.powercastco.com/ states that it can be higher or lower.
} 

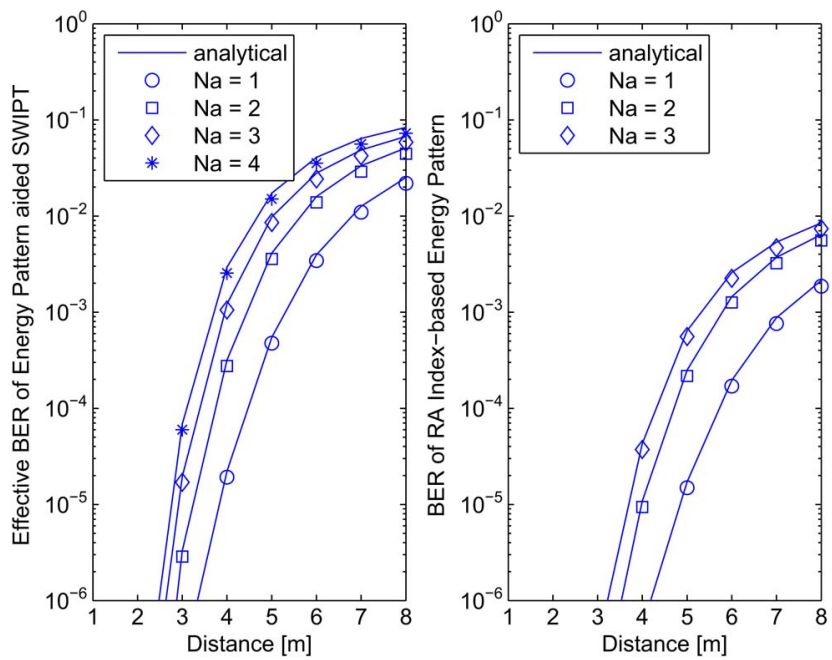

Fig. 2. Analytical and simulated performance of a $\left\{N_{t}, N_{r}\right\}=\{8,4\}$ energy pattern aided SWIPT system, characterizing both the effective BER of the energy pattern (left) and the BER of the RA index-based energy pattern in isolation (right). In addition, we assume $M$-ary intensity-based energy pattern using the equi-spaced amplitudes $\mathcal{A}_{E A}$ and experiencing Rayleigh fading with $\rho<1$.
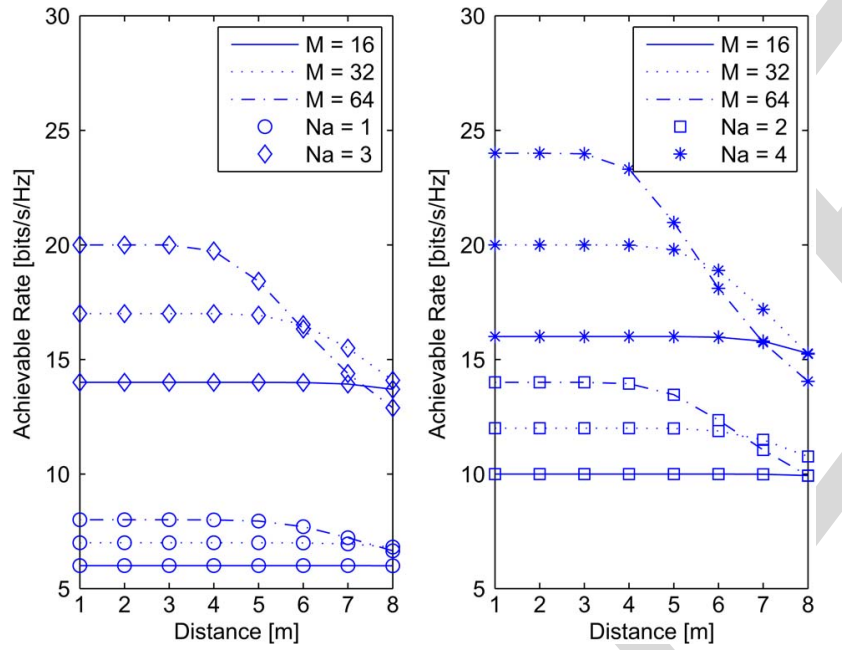

Fig. 3. Achievable rate of a $\left\{N_{t}, N_{r}\right\}=\{8,4\}$ energy pattern aided SWIPT system employing various $M$-ary intensity-based energy pattern using the equispaced amplitudes $\mathcal{A}_{E A}$ and experiencing Rayleigh fading with $\rho<1$.

with $\rho<1$. It may be seen from Fig. 3 that the higher the value of $N_{a} / N_{t}$, the higher the attainable rate becomes. Importantly, by employing RA index-based information transfer, extra SD symbols are also conveyed. When investigating a fixed $N_{a}$, $M$-ary energy pulse based symbols are preferred from an achievable rate perspective, when the receiver is sufficiently close to the transmitter. Naturally, $M$ has to be gradually reduced, when the receiver is further apart from the TA. This suggests that a distance-dependent adaptive energy pattern regime may be conceived for our future work.

3) Power Transfer: Fig. 4 shows the power conversion of an $\left\{N_{t}, N_{r}\right\}=\{8,4\}$ (left) and of an $\left\{N_{t}, N_{r}\right\}=\{4,2\}$ (right) energy pattern aided SWIPT system employing $M$-ary intensitybased energy patterns using the equi-spaced amplitudes $\mathcal{A}_{E A}$ and experiencing Rician fading associated with $K=[0,1,3,5]$ and $\rho=1$, where both the CI-TPC aided arrangement and
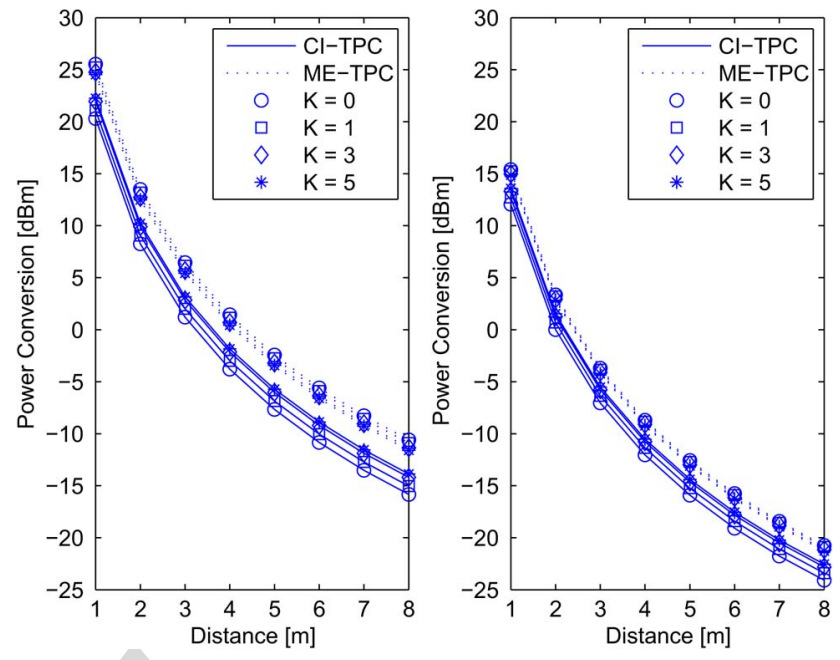

Fig. 4. Power conversion of a $\left\{N_{t}, N_{r}\right\}=\{8,4\}$ (left) and $\left\{N_{t}, N_{r}\right\}=\{16,8\}$ (right) energy pattern aided SWIPT system experiencing employing $M$-ary intensity-based energy patterns using the equi-spaced amplitudes $\mathcal{A}_{E A}$ and experiencing Rician fading associated with $K=[0,1,3,5]$ and $\rho=1$, where both the CI-TPC aided arrangement and the ME-TPC aided arrangement are included.

the ME-TPC aided arrangement are included, since the latter is capable of maximising the attainable power conversion. Observe by comparing the left and right subplot of Fig. 3 that the higher the number of TAs and RAs, the higher the converted power and the higher the performance-discrepancy between the CI-TPC and the ME-TPC aided arrangements.

Another interesting finding is that the higher the Rician $K$-factor, the higher the converted power of the CI-TPC aided arrangement. This is because when CI is used for our energy pattern aided SWIPT system, the reduced power conversion experienced is due to the detrimental effect of CI, when the channel is more dramatically faded. Thus, a high value of $K$ implies more benign LOS propagation, leading to an increased power conversion. On the other hand, the higher the value of $K$, the lower the converted power for the ME-TPC aided arrangement. This is because a higher value of $K$ leads to a reduced ratio between the maximum and minimum eigenvalues. Finally, the performance-dependence of the CI-TPC aided arrangement on $K$ is more obvious than that of the ME-TPC aided arrangement.

\section{Trade-Offs Results}

1) Trade-Offs: Fig. 5 shows the trade-offs between the achievable rate as well as power conversion of a $\left\{N_{t}, N_{r}\right\}=$ $\{8,4\}$ energy pattern aided SWIPT system employing $M$-ary intensity-based energy patterns using the equi-spaced amplitudes $\mathcal{A}_{E A}$ and experiencing Rician fading associated with $K=$ $[0,1,3,5]$ at $d_{0}=5 \mathrm{~m}$, where both the CI-TPC aided arrangement and the ME-TPC aided arrangement are characterised.

Let us now investigate the so-called trade-off bound for each $N_{a}$, which consists of a horizontal line segment, a sloping diagonal line segment and a vertical line segment. Firstly, since Proposition II.1 and II.2 implies that the power conversion of the CI-TPC aided arrangement does not affect the achievable rate, this is explicitly illustrated by the horizontal line segment 


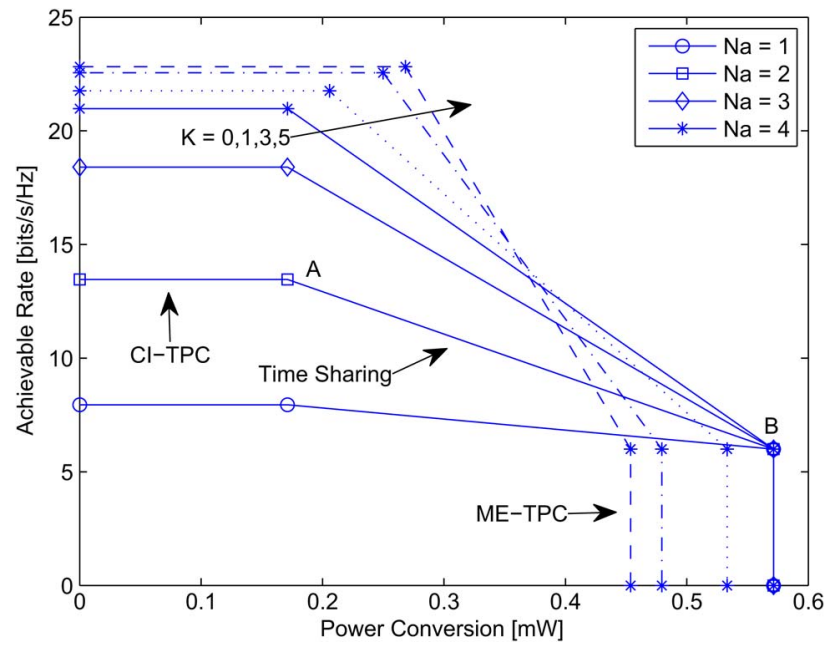

Fig. 5. Trade-offs between the achievable rate and power conversion of a $\left\{N_{t}, N_{r}\right\}=\{8,4\}$ energy pattern aided SWIPT system employing $M$-ary intensity-based energy patterns using the equi-spaced amplitudes $\mathcal{A}_{E A}$ and experiencing Rician fading associated with $K=[0,1,3,5]$ at $d_{0}=5 \mathrm{~m}$, where both the CI-TPC aided arrangement and the ME-TPC aided arrangement are characterised.

associated with all the legends corresponding to different $N_{a}$ values. This implies that if the required power conversion is less than A, as shown in Fig. 5, then employing the CI-TPC aided arrangement would result in a high achievable rate. On the other hand, when ME-TPC aided arrangement is considered, the maximum power conversion may be achieved in conjunction with a rate of $6 \mathrm{bits} / \mathrm{s} / \mathrm{Hz}$, since only a single intensity-based information stream is allowed. This is illustrated by the vertical line segment, implying that when the achievable rate is lower than B, as shown in Fig. 5, then employing the ME-TPC aided arrangement would achieve a high power conversion. Finally, the remaining segment of the trade-off bound may be characterised by the sloping diagonal line segment connecting the bounds of the CI-TPC aided arrangement and the METPC aided arrangement, where a time-sharing strategy may be employed, as indicated in Fig. 5.

Two further observations emerge from the Fig. 5. Firstly, the higher the ratio $N_{a} / N_{r}$, the larger the area under the trade-off bound. Secondly, as $K$ increases, having a less severely faded channel leads to a higher achievable rate and a higher power conversion. Thus we observe an enlarged trend of the trade-off bound towards the CI-TPC aided arrangement. By contrast, we have a shrinking trend of the trade-off bound towards the METPC aided arrangement. This is because a higher value of $K$ leads to a reduced ratio between the maximum and minimum eigenvalues.

2) Comparisons: The left subplot of Fig. 6 shows the tradeoffs between the goodput and power conversion of a $\left\{N_{t}, N_{r}\right\}=$ $\{8,4\}$ energy pattern aided SWIPT system employing $M$-ary intensity-based energy patterns using the equi-spaced amplitudes $\mathcal{A}_{E A}$ and experiencing Rayleigh fading at $d_{0}=5 \mathrm{~m}$, where both the CI-TPC aided arrangement and the ME-TPC aided arrangement are characterised. Using the achievable rate as our metric as in Fig. 5 partially conceals the fact that the MIB for a low value of $N_{a}$ is actually higher than that of a high $N_{a}$, as demonstrated by the BER results in the right subplot of Fig. 2 .
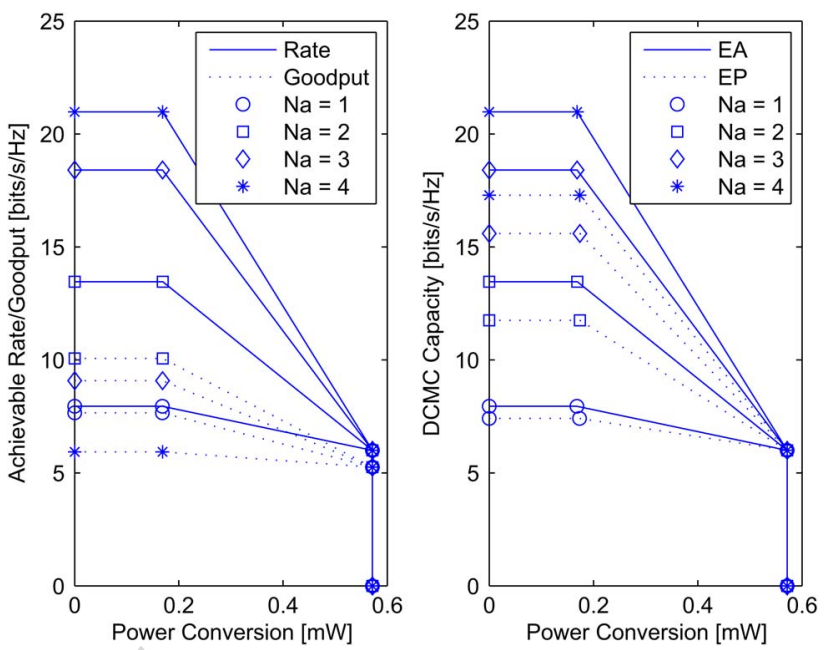

Fig. 6. Trade-off between the goodput and power conversion (right) and between the achievable rate and power conversion when $\mathcal{A}_{E P}$ is used instead of $\mathcal{A}_{E A}$. In both subplots, a $\left\{N_{t}, N_{r}\right\}=\{8,4\}$ energy pattern aided SWIPT system is considered, where both the CI-TPC aided arrangement and the METPC aided arrangement are characterised for an $M$-ary intensity-based energy pattern at $d_{0}=5 \mathrm{~m}$ and experiencing Rayleigh fading.

Thus, apart from the achievable rate, the reliability of each transmitted bit is also important. We defined the goodput as $1-\left(1-e_{b}^{e}\right)^{L}$, where $L$ represents the frame length, which is set to $L=100$. This may also be referred to as the BLock Error Ratio (BLER) indicating the Quality of Service (QoS), where we may observe that in contrast to using the achievable rate as our metric, it is no longer true that a higher $N_{a} / N_{r}$ value leads to a larger area under the trade-off bound.

The right subplot of Fig. 6 characterizes the trade-off between the achievable rate and the power conversion of a $\left\{N_{t}, N_{r}\right\}=\{8,4\}$ SWIPT system employing $M$-ary intensitybased energy patterns using either the equi-spaced amplitudes $\mathcal{A}_{E A}$ or the equi-spaced power $\mathcal{A}_{E P}$ and experiencing Rayleigh fading at $d_{0}=5 \mathrm{~m}$, where both the CI-TPC aided arrangement and the ME-TPC aided arrangement are characterised. It is clearly shown in the right subplot of Fig. 6 that the EA design of intensity-based energy pattern exhibits a higher achievable rate, while maintaining the same level of power conversion as the EP design. However, this is achieved at the cost of exhibiting a higher Peak to Average Power Ratio (PAPR).

\section{CONCLUSION}

A novel energy pattern aided SWIPT system was proposed, where both RA index-based information transfer as well as intensity-based information transfer were employed in the context of both the integrated receiver mode and the power-split mode of Fig. 1, where two-stage detection algorithms were used. The analytical BER of both the SD symbols, as well as of the energy pulse based symbols and of the overall energy pattern were also derived. Both the analytical and simulation results demonstrate that our energy pattern aided SWIPT system exhibits a beneficial immunity to power conversion. Our proposed energy pattern based SWIPT system leads to a beneficial wireless information and power transfer convergence, where the achievable rate versus power conversion trade-off bounds 
and the goodput versus power conversion trade-off bounds were also discussed. Hence, the energy pattern aided SWIPT system may be viewed as an instantiation of joint information and energy transfer in the spirit of Varshney's seminal concept [7], where information and energy transfer are inter-linked.

In fact, our conclusions concerning the immunity to power conversion revealed in our propositions are idealized. Practically, $\rho$ cannot be tuned to be asymptotically close to unity, since a certain minimum required power would be reserved for information transfer, where the amount of reserved power depends on the sensitivity of the practical detection circuit. Moreover, the information transfer performance would not be independent of $\rho$ owing to the finite processing accuracy of the practical detection circuit, which exhibits its own imperfections and limitations as a function of the incident power. Hence, a promising future research topic would be to exploit the flexibility of our design by adapting the choice of $N_{a}$ and by balancing it along the trade-off curves with the above-mentioned practical aspects explicitly considered.

\section{APPENDIX A}

\section{PROOF OF LEMMA II.1}

Let us assume that the first-stage detection of (19) suggested that the RA pattern $\hat{k}$ was activated. Then, upon considering the $\hat{v}_{i}=\mathcal{C}(\hat{k}, i)$ th activated RA, the ML detector determines the most likely energy pulse based symbols $b_{n_{i}} \in \mathcal{A}$ that results into the observation of $\left|y_{\hat{v}}\right|^{2}$ in (18), when we have equi-probable $b_{n_{i}}$ values.

Assuming that $b_{n_{i}}$ was transmitted, we have the observation given by

$$
\begin{aligned}
G & =(1-\rho)\left|\sqrt{\beta / N_{a}} b_{n_{i}}+w_{\hat{v}_{i}}\right|^{2}, \\
& =(1-\rho)\left[\left(\sqrt{\beta / N_{a}} b_{n_{i}}+\mathcal{R}\left\{w_{\hat{v}_{i}}\right\}\right)^{2}+\mathcal{J}\left\{w_{\hat{v}_{i}}\right\}^{2}\right] .
\end{aligned}
$$

By normalising (35) with respect to $(1-\rho) \sigma_{0}^{2}$, it is readily seen that $g=G /(1-\rho) \sigma_{0}^{2}$ obeys

$$
g \sim\left[\mathcal{N}\left(\sqrt{\lambda} b_{n_{i}}, 1\right)\right]^{2}+[\mathcal{N}(0,1)]^{2}=\chi_{2}^{2}\left(g ; \lambda b_{n_{i}}^{2}\right)
$$

where $\lambda=\beta / N_{a} \sigma_{0}^{2}$. Thus, it is clear that the likelihood of $b_{n_{i}}$ that results into the observation of $A=\left|y_{\hat{v}_{i}}\right|^{2} /(1-\rho) \sigma_{0}^{2}=$ $\left|r_{\hat{v}_{i}}\right|^{2} / \sigma_{0}^{2}$ is

$$
\mathcal{L}=f_{\chi_{2}^{2}}\left(g ; \lambda b_{n_{i}}^{2}\right)_{g=A} .
$$

As a result, the ML detection criterion is given by (21).

\section{APPENDIX B \\ PROOF OF LEMMA II.2}

Let $\mathcal{A}_{k_{p}}$ denote the alphabet of the SD symbol having $k_{p}$ bits of information. Then the cardinality of the alphabet $\mathcal{A}_{k_{p}}$ is twice higher compared to that of $\mathcal{A}_{k_{p}-1}$. Thus, $\mathcal{A}_{k_{p}}$ may be constructed by a pair of sub-alphabets of $\mathcal{A}_{k_{p}-1}$, represented by 0 and 1, respectively. We may thereafter refer to the alphabet of $\mathcal{A}_{k_{p}-1}$ preceded by the above-mentioned 0 as the zero-alphabet, while the other one as the one-alphabet.
Assuming that the SD symbol representing $k_{p}$ zeros was transmitted, we may then calculate the total number of pairwise bit errors $\varepsilon_{0}$ in the above-mentioned zero-alphabet. Hence, the number of pair-wise bit errors $\varepsilon_{1}$ in the one-alphabet is simply $\varepsilon_{1}=\varepsilon_{0}+2^{k_{p}}$, which accounts for the difference in the first preceding bit. Hence the total number of pair-wise bit errors is $\varepsilon=2 \varepsilon_{0}+2^{k_{p}}$. Assuming an equal probability of $1 /\left(2^{k_{p}}-1\right)$ for each possible SD symbol error, we arrive at the correction factor given by $\delta_{k_{p}}=\left(2 \varepsilon_{0}+2^{k_{p}}\right) /\left(2^{k_{p}}-1\right)$.

Since $\varepsilon_{0}$ represents the total number of pair-wise bit errors corresponding to the case of $\left(k_{p}-1\right)$ bits of information, we have $\varepsilon_{0}=\left(2^{k_{p}-1}-1\right) \delta_{k_{p}-1}$. Hence the resultant expression of the correction factor may be calculated recursively according to (27) after some further manipulations.

\section{APPENDIX C}

\section{PROOF OF LEMMA II. 3}

Considering the general case of $N_{r}$ as well as $N_{a}$ and assuming that the RA pattern $\mathcal{C}(k)$ was activated, according to Proposition II.1 and dropping $\rho$, for each metric of (18), we have for $v_{i} \in \mathcal{C}(k)$ and $u_{j} \in \overline{\mathcal{C}}(k)$ :

$$
r_{v_{i}}=\sqrt{\beta / N_{a}} b_{m_{i}}+w_{v_{i}} ; \quad r_{u_{j}}=w_{u_{j}}
$$

where $\overline{\mathcal{C}}(k)$ denotes the complementary set of the activated RA pattern $\mathcal{C}(k)$ in $\mathcal{C}$. Upon introducing $\sigma_{0}^{2}=\sigma^{2} / 2$, we have:

$$
\begin{aligned}
\left|r_{y_{i}}\right|^{2} & \sim\left[\mathcal{N}\left(\sqrt{\beta / N_{a}} b_{m_{i}}, \sigma_{0}^{2}\right)\right]^{2}+\left[\mathcal{N}\left(0, \sigma_{0}^{2}\right)\right]^{2} \\
\left|r_{u_{j}}\right|^{2} & \sim\left[\mathcal{N}\left(0, \sigma_{0}^{2}\right)\right]^{2}+\left[\mathcal{N}\left(0, \sigma_{0}^{2}\right)\right]^{2}
\end{aligned}
$$

By normalising with respect to $\sigma_{0}^{2}$, we observe that of $g_{v_{i}}=$ $\left|r_{v_{i}}\right|^{2} / \sigma_{0}^{2}$ and $g_{u_{j}}=\left|r_{u_{j}}\right|^{2} / \sigma_{0}^{2}$ obey

$$
g_{v_{i}} \sim \chi_{2}^{2}\left(g ; \lambda b_{m_{i}}^{2}\right) ; \quad g_{u_{j}} \sim \chi_{2}^{2}(g)
$$

where $\lambda=\beta / N_{a} \sigma_{0}^{2}$.

Recall from (20) that the correct decision concerning the SD symbols occurs, when $\sum_{i=1}^{N_{a}}\left|r_{v_{i}}\right|^{2}$ attains its maximum. By expressing $N_{a}^{\prime}=\left(N_{r}-N_{a}\right)$, the lower bound of the correct detection probability $\bar{e}_{s, l b}^{p}\left(\lambda, \boldsymbol{b}_{m}\right)$ of the SD symbols may be expressed as in (42), shown at the top of the next page, when the energy pattern based symbols $\boldsymbol{b}_{m}$ were transmitted and the RA pattern $\mathrm{C}(k)$ was activated. To elaborate further, Equation (42-a) serves as the lower bound, since it sets the most strict condition for the correct detection, when each metric $g_{u_{j}}$ of the inactivated RA indices in $\overline{\mathrm{C}}(k)$ is lower than each metric $g_{v_{i}}$ of the activated RA indices in $\mathcal{C}(k)$, while Equation (42-b) follows from the fact that the $N_{a}$ random variables $g_{v_{i}}$ are independent of each other. Furthermore, Equation (42-c) follows from the fact that the $N_{a}^{\prime}$ random variables $g_{u_{j}}$ are independent. After averaging over $\mathcal{C}(k)$, we arrive at expression of (29), since $\mathbb{E}_{\mathcal{C}(k)}\left[\bar{e}_{s, l b}^{p}\left(\lambda, \boldsymbol{b}_{m}\right)\right]=\bar{e}_{s, l b}^{p}\left(\lambda, \boldsymbol{b}_{m}\right)$. As a result, after averaging further over the distribution of $f_{\lambda}(\lambda)$ and taking the expectation over the combinations of $\boldsymbol{b}_{m}$, the analytical SER $e_{s}^{p}$ of the SD symbol may be upper bounded as in (28). In general, $f_{\lambda}(\lambda)$ can be acquired with the aid of the empirical histogram based method. 


$$
\begin{aligned}
\bar{e}_{s, l b}^{p}\left(\lambda, \boldsymbol{b}_{m}\right) & \stackrel{a}{=} \int_{0}^{\infty} P\left(g_{u_{1}}<g_{v_{1}}, \ldots, g_{u_{N_{a}^{\prime}}}<g_{v_{1}}, \ldots, g_{u_{1}}<g_{v_{N_{a}}}, \ldots, g_{u_{N_{a}^{\prime}}}<g_{v_{N_{a}}}\right) P\left(g_{v_{1}}, \ldots, g_{v_{N_{a}}} \mid \lambda, \boldsymbol{b}_{m}\right) d g_{v_{1}} \cdots d g_{v_{N_{a}}} \\
& \stackrel{b}{=} \prod_{i=1}^{N_{a}} \int_{0}^{\infty} P\left(g_{u_{1}}<g_{v_{i}}, \ldots, g_{u_{N_{a}^{\prime}}}<g_{v_{i}}\right) P\left(g_{v_{i}} \mid \lambda, b_{m_{i}}\right) d g_{v_{i}} \\
& \stackrel{c}{=} \prod_{i=1}^{N_{a}} \int_{0}^{\infty} \prod_{u_{j} \in \overline{\mathcal{C}}(k)} P\left(g_{u_{j}}<g_{v_{i}}\right) P\left(g_{v_{i}} \mid \lambda, b_{m_{i}}\right) d g_{v_{i}}=\prod_{i=1}^{N_{a}} \int_{0}^{\infty}\left[F_{\chi_{2}^{2}}(g)\right]^{N_{r}-N_{a}} f_{\chi_{2}^{2}}\left(g ; \lambda b_{m_{i}}^{2}\right) d g
\end{aligned}
$$

\section{APPENDIX D \\ PROOF OF LEMMA II.4}

Since the stage-one detection of (19) significantly affects the stage-two detection of (21), we first introduce the SER $\tilde{e}_{s}^{s}$ of the energy pulse symbols for intensity-based information transfer in the absence of SD symbol errors. By considering (41), for a specific activated RA index $v_{i}=\mathrm{C}(k, i)$, the resultant decision metric is $g_{v_{i}} \sim \chi_{2}^{2}\left(g ; \lambda b_{m_{i}}^{2}\right)$. By dropping the index of $v_{i}$, the average SER becomes

$$
\tilde{e}_{s}^{s} \leq \int_{0}^{\infty} \frac{1}{M} \sum_{m=1}^{M} P_{e}\left(t_{m}, \lambda b_{m}^{2}\right) f_{\lambda}(\lambda) d \lambda=\tilde{e}_{s, u b}^{s}
$$

where for each error event $P_{e}\left(t_{m}, \lambda b_{m}^{2}\right)$, we have

$$
\begin{cases}1-F_{\chi_{2}^{2}}\left(g ; \lambda b_{1}^{2}\right)_{g=t_{1}}, & m=1 \\ F_{\chi_{2}^{2}}\left(g ; \lambda b_{M}^{2}\right)_{g=t_{M-1}}, & m=M \\ F_{\chi_{2}^{2}}\left(g ; \lambda b_{m}^{2}\right)_{g=t_{m-1}}+1-F_{\chi_{2}^{2}}\left(g ; \lambda b_{m}^{2}\right)_{g=t_{m}}, & \text { else }\end{cases}
$$

and the equality in (43) holds, when we set $t_{m}$ as the threshold, satisfying

$$
f_{\chi_{2}^{2}}\left(g ; \lambda b_{m}^{2}\right)_{g=t_{m}}=f_{\chi_{2}^{2}}\left(g ; \lambda b_{m+1}^{2}\right)_{g=t_{m}}
$$

while we may also derive the upper bound by simplifying it as $t_{m}=\lambda\left(b_{m}^{2}+b_{m+1}^{2}\right) / 2$, where $\lambda=\beta / N_{a} \sigma_{0}^{2}$.

Let us now consider the SER in the presence of SD symbol errors. The SER of $e_{s}^{s}$ is constituted by the SER of $\tilde{e}_{s}^{s}$, when the detection of the SD symbol is correct, having a probability of $\left(1-e_{s}^{p}\right)$ plus the SER, when the detection of the SD symbol is erroneous, having a probability of $e_{s}^{p}$, which is jointly expressed as

$$
\begin{aligned}
e_{s}^{s} & \leq\left(1-e_{s}^{p}\right) \tilde{e}_{s, u b}^{s}+e_{s}^{p} \sum_{\ell \neq k} P_{k \mapsto \ell} \underbrace{\frac{N_{c} \tilde{e}_{s, u b}^{s}+N_{d} e_{s}^{o}}{N_{a}}}_{E} \\
& <\left(1-e_{s}^{p}\right) \tilde{e}_{s, u b}^{s}+\frac{e_{s}^{p}}{\left(2^{k_{p}}-1\right)} \sum_{\ell \neq k} \frac{N_{c} \tilde{e}_{s, u b}^{s}+N_{d} e_{s}^{o}}{N_{a}}, \\
& <\left(1-e_{s, u b}^{p}\right) \tilde{e}_{s, u b}^{s}+e_{s, u b}^{p} \frac{\sum_{\ell \neq k} N_{c} \tilde{e}_{s, u b}^{s}+N_{d} e_{s}^{o}}{N_{a}\left(2^{k_{p}}-1\right)} .
\end{aligned}
$$

Regarding the second additive part of (44), the true activated RA pattern $\mathrm{C}(k)$ may be erroneously deemed to be any of the other legitimate RA patterns $\mathcal{C}(\ell) \in \mathcal{C}, \ell \neq k$ with a probability of $P_{k \mapsto \ell}$, which we have to average over. As for the calculation of the per-case error rates $E$, when $\mathcal{C}(k)$ was erroneously detected as a particular $\mathcal{C}(\ell)$, we found that it was constituted by the error rates of $\tilde{e}_{s, u b}^{s}$ for those $N_{c}$ RAs in common (which maybe regarded as being partially correctly detected) and the error rates of $e_{s}^{o}$ for those RAs that were exclusively hosted by $\mathrm{C}(\ell)$, but were excluded from $\mathcal{C}(k)$. Furthermore, since only random noise may be received by those $N_{d}$ RAs in $\mathrm{C}(\ell)$, thus $e_{s}^{o}$ simply represents the SER of the energy pulse symbols as a result of a random guess, i.e. we have $e_{s}^{o}=(M-1) / M$.

Although it is natural that energy patterns with a higher $N_{c}$ would be more likely to cause a erroneous detection, we assume an equal probability of $P_{k \mapsto \ell}=1 /\left(2^{k_{p}}-1\right)$. Since naturally, we have $e_{s}^{o} \geq \tilde{e}_{s, u b}^{s}$, the equal probability assumption leads to the relation of (45). Furthermore, we also have $E \geq \tilde{e}_{s, u b}^{s}$. As a result, (46) holds, since upon replacing $e_{s}^{p}$ by $e_{s, u b}^{p}$ puts more weights on the second additive part, which is more prone to errors.

\section{REFERENCES}

[1] K. Maruyama, F. Nori, and V. Vedral, "The physics of Maxwell's demon and information," Rev. Modern Phys., vol. 81, pp. 1-23, Jan. 2009.

[2] R. Landauer, "Irreversibility and heat generation in the computing process,” IBM J. Res. Develop., vol. 5, no. 3, pp. 183-191, Jul. 1961.

[3] M. Saeedi and I. L. Markov, "Synthesis and optimization of reversible circuits-A survey," ACM Comp. Surveys, vol. 45, no. 2, pp. 1-34, Mar. 2013

[4] S. Toyabe, T. Sagawa, M. Ueda, E. Muneyuki, and M. Sano, "Experimental demonstration of information-to-energy conversion and validation of the generalized Jarzynski equality," Nat. Phys., vol. 6, pp. 988-992, Nov. 2010.

[5] O. L. de Weck, D. Roos, and C. L. Magee, Engineering Systems: Meeting Human Needs in a Complex Technological World. Cambridge, MA, USA: MIT Press, 2011.

[6] L. Varshney, "Transporting information and energy simultaneously," in Proc. IEEE Int. Symp. Inf. Theory, 2008, pp. 1612-1616.

[7] L. Varshney, "On energy/information cross-layer architectures," in Proc. IEEE Int. Symp. Inf. Theory, 2012, pp. 1356-1360.

[8] P. Grover and A. Sahai, "Shannon meets Tesla: Wireless information and power transfer," in Proc. IEEE Int. Symp. Inf. Theory, 2010, pp. 2363 2367.

[9] N. Tesla, "The future of the wireless art," in Proc. Wireless Telegraphy Telephony, 1908, pp. 67-71.

[10] J. Garnica, R. Chinga, and J. Lin, "Wireless power transmission: From far-field to near-field," Proc. IEEE, vol. 101, no. 6, pp. 1321-1331, Jun. 2013.

[11] A. Kurs et al., "Wireless power transfer via strongly coupled magnetic resonances," Science, vol. 317, no. 5834, pp. 83-86, Jul. 2007.

[12] W. C. Brown, "The history of power transmission by radio waves," IEEE Trans. Microw. Theory Techn., vol. 32, no. 9, pp. 1230-1242, Sep. 1984.

[13] W. C. Brown and E. Eves, "Beamed microwave power transmission and its application to space," IEEE Trans. Microw. Theory Techn., vol. 40, no. 6, pp. 1239-1250, Jun. 1992. 
[14] J. McSpadden and J. Mankins, "Space solar power programs and microwave wireless power transmission technology," IEEE Microw. Mag., vol. 3, no. 4, pp. 46-57, Dec. 2002.

[15] J. A. G. Akkermans, M. van Beurden, G. J. N. Doodeman, and H. Visser, "Analytical models for low-power rectenna design," IEEE Antennas Wireless Propag. Lett., vol. 4, pp. 187-190, Jun. 2005.

[16] A. Massa, G. Oliveri, F. Viani, and P. Rocca, "Array designs for longdistance wireless power transmission: State-of-the-art and innovative solutions," Proc. IEEE, vol. 101, no. 6, pp. 1464-1481, Jun. 2013.

[17] A. M. Hawkes, A. R. Katko, and S. A. Cummer, "A microwave metamaterial with integrated power harvesting functionality," Appl. Phys. Lett., vol. 103, no. 16, Oct. 2013, Art. ID. 163901.

[18] A. Osseiran et al., "Scenarios for the 5G mobile and wireless communications: The vision of the METIS project," IEEE Commun. Mag., vol. 52, no. 5, May 2014.

[19] T. Taleb and A. Kunz, "Machine type communications in 3GPP networks: Potential, challenges, solutions," IEEE Commun. Mag., vol. 50, no. 3, pp. 178-184, Mar. 2012.

[20] L. Atzori, A. Iera, and G. Morabito, "The Internet of things: A survey," Comput. Netw., vol. 54, no. 15, pp. 2787-2805, Oct. 2010.

[21] R. Zhang and C. K. Ho, "MIMO broadcasting for simultaneous wireless information and power transfer," IEEE Trans. Wireless Commun., vol. 12, no. 5, pp. 1989-2001, May 2013.

[22] J. Park and B. Clerckx, "Joint wireless information and energy transfer in a two-user MIMO interference channel," IEEE Trans. Wireless Commun., vol. 12, no. 8, pp. 4210-4221, Aug. 2013.

[23] A. Fouladgar and O. Simeone, "On the transfer of information and energy in multi-user systems," IEEE Commun. Lett., vol. 16, no. 11, pp. 1733-1736, Nov. 2012.

[24] A. Fouladgar and O. Simeone, "Information and energy flows in graphical networks with energy transfer and reuse," IEEE Wireless Commun. Lett., vol. 2, no. 4, pp. 371-374, Aug. 2013.

[25] L. Liu, R. Zhang, and K.-C. Chua, "Secrecy wireless information and power transfer with MISO beamforming," IEEE Trans. Signal Process., vol. 62, no. 7, pp. 1850-1863.

[26] L. Liu, R. Zhang, and K.-C. Chua, "Wireless information transfer with opportunistic energy harvesting," IEEE Trans. Wireless Commun., vol. 12, no. 1, pp. 288-300, Jan. 2013.

[27] L. Liu, R. Zhang, and K.-C. Chua, "Wireless information and power transfer: A dynamic power splitting approach," IEEE Trans. Commun., vol. 61, no. 9, pp. 3990-4001, Sep. 2013.

[28] X. Zhou, R. Zhang, and C. K. Ho, "Wireless information and power transfer: Architecture design and rate-energy tradeoff," IEEE Trans. Commun., vol. 61, no. 11, pp. 4757-4767, Nov. 2013.

[29] K. Huang and E. G. Larsson, "Simultaneous information-and-power transfer for broadband downlink systems," IEEE Trans. Signal Process., vol. 61, no. 23, pp. 5972-5986, Dec. 2013.

[30] H. Ju and R. Zhang, "Throughput maximization for wireless powered communication networks," IEEE Trans. Wireless Commun., vol. 13, no. 1, pp. 418-428, Jan. 2014.

[31] V. Chawla and D.-S. Ha, "An overview of passive RFID," IEEE Commun. Mag., vol. 45, no. 9, pp. 11-17, Sep. 2007.

[32] P. Popovski, A. Fouladgar, and O. Simeone, "Interactive joint transfer of energy and information," IEEE Trans. Commun., vol. 61, no. 5, pp. 2086-2097, May 2013.

[33] B. Gurakan, O. Ozel, J. Yang, and S. Ulukus, "Energy cooperation in energy harvesting communications," IEEE Trans. Commun., vol. 61, no. 12, pp. 4884-4898, Dec. 2013.

[34] D. Ng, E. Lo, and R. Schober, "Wireless information and power transfer: Energy efficiency optimization in OFDMA systems," IEEE Trans. Wireless Commun., vol. 12, no. 12, pp. 6352-6370, Dec. 2013.

[35] X. Zhou, R. Zhang, and C. K. Ho, "Wireless information and power transfer in multiuser OFDM systems," IEEE Trans. Wireless Commun., vol. 13, no. 4, pp. 2282-2294, Apr. 2014.

[36] I. Krikidis, S. Timotheou, and S. Sasaki, "RF energy transfer for cooperative networks: Data relaying or energy harvesting?" IEEE Commun. Lett., vol. 16, no. 11, pp. 1772-1775, Nov. 2012.

[37] Z. Xiang and M. Tao, "Robust beamforming for wireless information and power transmission," IEEE Wireless Commun. Lett., vol. 1, no. 4, pp. 372375, Aug. 2012.

[38] X. Chen, C. Yuen, and Z. Zhang, "Wireless energy and information transfer tradeoff for limited feedback multi-antenna systems with energy beamforming," IEEE Trans. Veh. Technol., vol. 63, no. 1, pp. 407-412, Jan. 2014.

[39] X. Zhou, X. Zheng, R. Zhang, and L. Hanzo, "Chip-interleaved optical code division multiple access relying on a photon-counting iterative suc- cessive interference canceller for free-space optical channels," Opt. Exp., vol. 21, no. 13, pp. 15926-15937, Jul. 2013.

[40] L. Li, X. Zhou, R. Zhang, and L. Hanzo, "Performance and capacity analysis of Poisson photon-counting based Iter-PIC OCDMA systems," Optics Exp., vol. 21, no. 22, pp. 25 954-25 967, Nov. 2013.

[41] R. Zhang, L.-L. Yang, and L. Hanzo, "Generalised pre-coding aided spatial modulation," IEEE Trans. Wireless Commun., vol. 12, no. 11, pp. 5434-5443, Nov. 2013.

[42] R. Zhang, L.-L. Yang, and L. Hanzo, "Error probability and capacity analysis of generalised pre-coding aided spatial modulation," IEEE Trans. Wireless Commun., vol. 14, no. 1, pp. 364-375, Jan. 2015. [Online]. Available: http://ieeexplore.ieee.org/stamp/stamp.jsp?arnumber=6877673

[43] C. Peel, B. Hochwald, and A. Swindlehurst, "A vector-perturbation technique for near-capacity multiantenna multiuser communication-Part I: Channel inversion and regularization," IEEE Trans. Commun., vol. 53, no. 1, pp. 195-202, Jan. 2005.

[44] Q. Spencer, A. Swindlehurst, and M. Haardt, "Zero-forcing methods for downlink spatial multiplexing in multiuser MIMO channels," IEEE Trans. Signal Process., vol. 52, no. 2, pp. 461-471, Feb. 2004.

[45] H. Urkowitz, "Energy detection of unknown deterministic signals," Proc. IEEE, vol. 55, no. 4, pp. 523-531, Apr. 1967.

[46] M. D. Renzo, H. Haas, A. Ghrayeb, S. Sugiura, and L. Hanzo, "Spatial modulation for generalized MIMO: Challenges, opportunities and implementation," Proc. IEEE, vol. 102, no. 1, pp. 56-103, Jan. 2014. [Online]. Available: http://eprints.soton.ac.uk/354175/

[47] R. Abu-alhiga and H. Haas, "Subcarrier-index modulation OFDM," in Proc. IEEE Int. Symp. Pers., Indoor Mobile Radio Commun., Sep. 2009, pp. $177-181$.

[48] F. Ellinger et al., "Integrated adjustable phase shifters," IEEE Microw. Mag., vol. 11, no. 6, pp. 97-108, Oct. 2010.

[49] R. Eickhoff, R. Kraemer, I. Santamaria, and L. Gonzalez, "Developing energy-efficient MIMO radios," IEEE Veh. Technol. Mag., vol. 4, no. 1, pp. 34-41, Mar. 2009.

[50] F. Ellinger, U. Jorges, U. Mayer, and R. Eickhoff, "Analysis and compensation of phase variations versus gain in amplifiers verified by $\mathrm{SiGe}$ HBT cascode RFIC," IEEE Trans. Microw. Theory Techn., vol. 57, no. 8, pp. 1885-1894, Aug. 2009.

[51] J. Via, I. Santamaria, V. Elvira, and R. Eickhoff, "A general criterion for analog Tx-Rx beamforming under OFDM transmissions," IEEE Trans. Signal Process., vol. 58, no. 4, pp. 2155-2167, Apr. 2010.

[52] R. Couillet and M. Debbah, Random Matrix Methods for Wireless Communications. New York, NY, USA: Cambridge Univ. Press, 2011.

[53] E. Karipidis, N. Sidiropoulos, and Z.-Q. Luo, "Far-field multicast beamforming for uniform linear antenna arrays," IEEE Trans. Signal Process., vol. 55 , no. 10 , pp. 4916-4927, Oct. 2007.

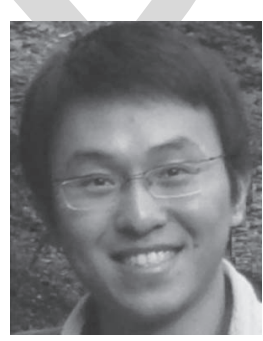

Rong Zhang (S'07-M'09) received the B.Sc. degree from Southeast University, Nanjing, China, in 2003 and the Ph.D. degree from Southampton University, Southampton, U.K., in 2009. Before receiving his Doctorate, he was an Engineer (August 2003-July 2004) at China Telecom and a Research Assistant (January 2006-May 2009) at Mobile Virtual Center of Excellence (MVCE), U.K. After being a Post-Doctoral Researcher (August 2009-July 2012) at Southampton University, he took an industrial consulting leave (August 2012-January 2013) for Huawei Sweden R\&D as a System Algorithms Specialist. Since February 2013, he has been a Lecturer in the CSPC group of ECS, Southampton University. He has $40+$ journals in prestigious publication avenues (e.g., IEEE, OSA) and many more in major conference proceedings. He regularly serves as reviewer for IEEE transactions/journals and has served several times as TPC member/invited session chair of major conferences. He is the recipient of joint funding from MVCE and EPSRC and is also a Visiting Researcher under Worldwide University Network (WUN). More details can be found at http://www.ecs.soton.ac.uk/people/rz. 


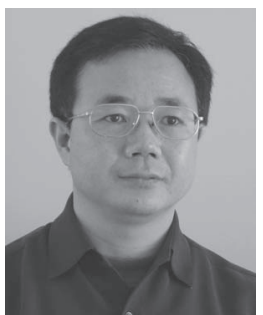

Lie-Liang Yang (M'98-SM'02) received the B.Eng. degree in communications engineering from Shanghai TieDao University, Shanghai, China, in 1988, and the M.Eng. and Ph.D. degrees in communications and electronics from Nothern (Beijing) Jiaotong University, Beijing, China, in 1991 and 1997, respectively. From June 1997 to December 1997, he was a Visiting Scientist with the Institute of Radio Engineering and Electronics, Academy of Sciences of the Czech Republic. Since December 1997, he has been with the University of Southampton, where he is the Professor of wireless communications in the School of Electronics and Computer Science. His research has covered a wide range of topics in wireless communications, networking and signal processing. He has published over 290 research papers in journals and conference proceedings, authored/co-authored three books, and also published several book chapters. The details about his publications can be found at http://www-mobile.ecs.soton.ac.uk/lly/. He is a fellow of the IET, served as an associate editor to the IEEE TRANSACTIONS ON VEHICULAR TECHNOLOGY and Journal of Communications and Networks (JCN), and is currently an associate editor to the IEEE Access and the Security and Communication Networks (SCN) Journal.

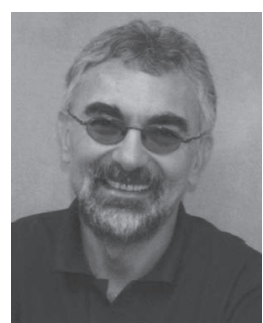

Lajos Hanzo (M'91-SM'92-F'08) received the degree in electronics in 1976 and the Doctorate degree in 1983. In 2009, he was awarded the honorary Doctorate "Doctor Honoris Causa" by the Technical University of Budapest. During his 38-year career in telecommunications he has held various research and academic posts in Hungary, Germany and the U.K. Since 1986, he has been with the School of Electronics and Computer Science, University of Southampton, U.K., where he holds the Chair in telecommunications. He has successfully supervised $80+\mathrm{Ph} . \mathrm{D}$. students, co-authored 20 John Wiley/IEEE Press books on mobile radio communications totalling in excess of 10000 pages, published $1400+$ research entries at IEEE Xplore, acted both as TPC and General Chair of IEEE conferences, presented keynote lectures and has been awarded a number of distinctions. Currently, he is directing a 100-strong academic research team, working on a range of research projects in the field of wireless multimedia communications sponsored by industry, the Engineering and Physical Sciences Research Council (EPSRC) U.K., the European Research Council's Advanced Fellow Grant and the Royal Society's Wolfson Research Merit Award. He is an enthusiastic supporter of industrial and academic liaison and he offers a range of industrial courses. He is also a Governor of the IEEE VTS. During 2008-2012, he was the Editor-in-Chief of the IEEE Press and a Chaired Professor also at Tsinghua University, Beijing. His research is funded by the European Research Council's Senior Research Fellow Grant. For further information on research in progress and associated publications please refer to http://www-mobile.ecs.soton.ac.uk Lajos has $20000+$ citations.

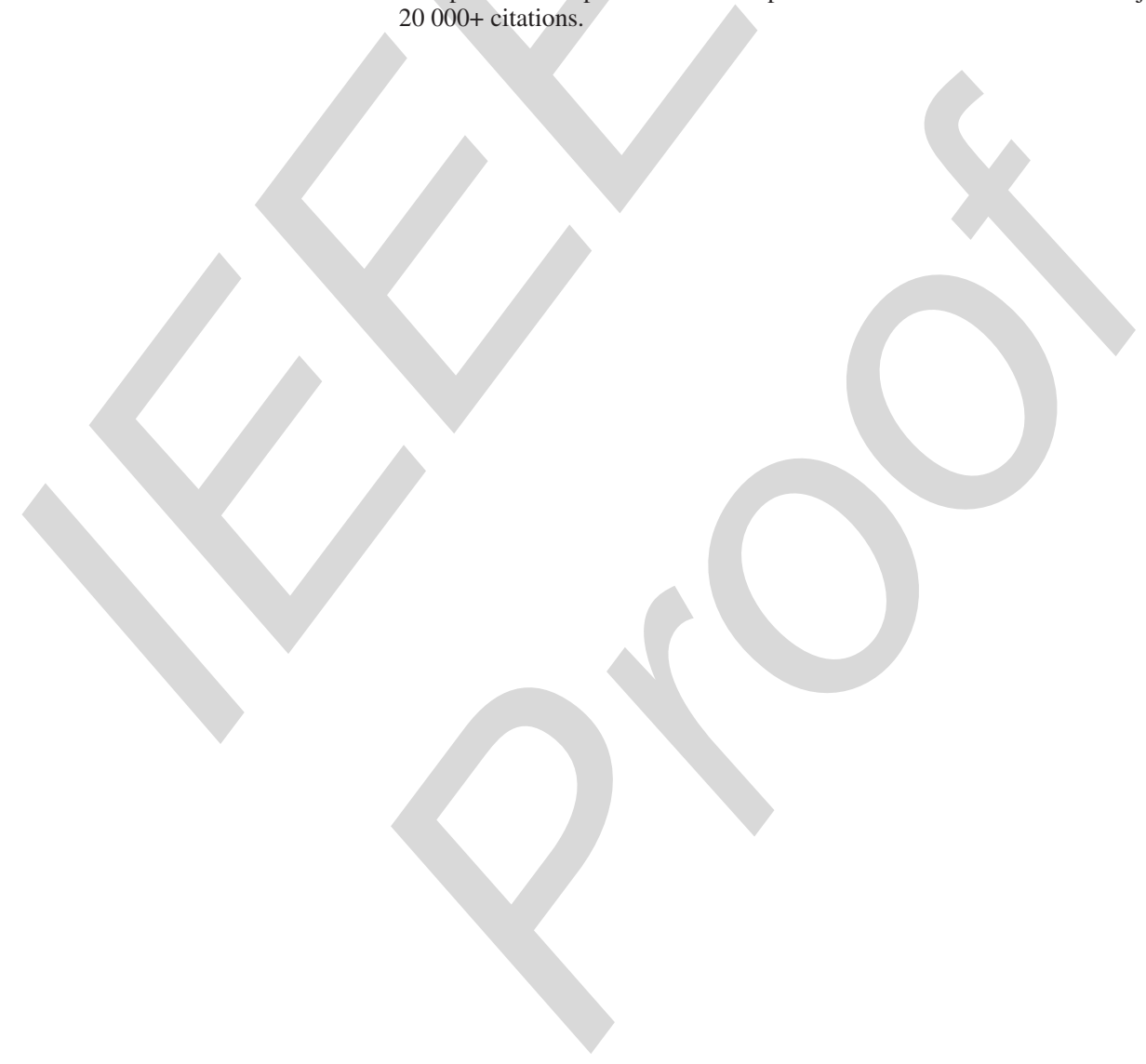




\title{
Energy Pattern Aided Simultaneous Wireless Information and Power Transfer
}

\author{
Rong Zhang, Member, IEEE, Lie-Liang Yang, Senior Member, IEEE, and Lajos Hanzo, Fellow, IEEE
}

\begin{abstract}
In echoing Varshney's seminal concept of jointly transmitting information and energy, we propose the concept of an energy pattern aided Simultaneous Wireless Information and Power Transfer (SWIPT) system, where in addition to power transfer, information is conveyed both by the specific Receive Antenna (RA) indices to which the power is delivered as well as by the particular intensity of the power assigned to that particular RA pattern. By embedding information into energy patterns rather than imposing it by modulating classic radio waveforms, our proposed solution is capable of operating both in an integrated receiver mode and in a power-split mode, while relying on a low-complexity two-stage non-coherent detection algorithm. Both our analysis and simulations show that our energy pattern aided SWIPT system exhibits a beneficial immunity to any potential performance degradation imposed by power-conversion. Moreover, the achievable rate versus power conversion trade-off bounds are characterized, demonstrating that our proposed energy pattern aided SWIPT system leads to a beneficial wireless information and power transfer convergence.
\end{abstract}

Index Terms-Simultaneous wireless information and power transfer, multiple input multiple output, transmitter pre-coding, pre-coded spatial modulation.

\section{INTRODUCTION}

1) Motivation: In statistical physics, there is a fundamentally under-explored and much-debated topic concerning information to energy conversion. The history of this subject has evolved from the early thought experiment of 1867 known as "Maxwell's Demon" [1] to the landmark philosophy referred to as "Landauer's principle" [2] in the mid-20th century as well as to the development of reversible electronics [3] and to the information heat engine [4] of our modern era. These seminal contributions suggested from a fundamental perspective the joint treatment of information and energy in future engineering systems [5]. Although it is a challenge to design practical systems benefiting from information to energy conversion, the explicit concept of transporting both information and energy simultaneously was also raised by the authors of [6], [7]. This topic was then further extended to the practical realms of wireless communications [8], since it is desirable to ensure

Manuscript received April 1, 2014; revised September 15, 2014; accepted December 16, 2014. The financial support of the EPSRC under the India-U.K. Advanced Technology Centre (IU-ATC), that of the EU under the Concerto project as well as that of the European Research Council's (ERC) Advanced Fellow Grant is gratefully acknowledged.

The authors are with the Southampton Wireless, School of ECS, University of Southampton, Southampton SO17 1BJ, U.K. (e-mail: rz@ecs.soton.ac.uk; 1ly@ecs.soton.ac.uk; lh@ecs.soton.ac.uk; http://www-mobile.ecs.soton.ac.uk).

Color versions of one or more of the figures in this paper are available online at http://ieeexplore.ieee.org.

Digital Object Identifier 10.1109/JSAC.2015.2391551 that a mobile device is entirely free from being tethered, as prophesied by Tesla a century ago [9]. Evolving from the above intriguing theoretical ideas, it is the maturing Wireless Power Transfer (WPT) and wireless communications fields that make the Simultaneous Wireless Information and Power Transfer (SWIPT) an important emerging research topic.

In the field of WPT, we refer the reader to a brief historical portrayal in [10]. Broadly speaking, WPT can be carried out in two basic ways, namely based on either electromagnetic induction in the form of inductive coupling and resonant coupling relying on coils [11] or with the aid of electromagnetic radiation using microwave frequencies by relying on so-called rectennas [12]. Early microwave power transmission was conceived in high-power applications in the Mega/Kilo-Watt-range in the context of the microwave-powered helicopter [13] and in the National Aeronautics and Space Administration's (NASA) feasibility study of the space-solar program [14]. Owing to the development of electronic devices in the late 20th century, research has been focused on the design of compact and efficient rectennas conceived for low-power applications in the milli/micro-Watt-range [15], [16], where the start-up company Powercast ${ }^{1}$ has reported that micro-Watt-scale power was transmitted over a distance of a few meters at the transmission power of $23 \mathrm{dBm}$ at a frequency around $900 \mathrm{MHz}$. Another state-of-the-art implementation was reported very recently in [17], which relied on a microwave meta-material that converts microwave energy into electric power at a conversion efficiency comparable to that of modern solar panels.

Owing to the fact that the electromagnetic radiation is restricted by both health and safety regulations, it remains an open challenge at the current state-of-the-art to power a mobile phone, typically requiring around hundreds of milliWatt power. Nonetheless, this technology is already appealing for employment in low-power sensors. Consequently, it becomes especially promising for the near-future wireless systems, where a compelling solution was proposed by the Mobile and wireless communications Enablers for the Twenty-twenty Information Society (METIS) project [18] with the goal of connecting billions of low-power devices globally. In this scenario, known as Machine Type Communications (MTC) [19] or the "Internet Of Things" (IOT) [20], SWIPT is expected to become a pervasive enabler to create the IoT, which is immortal from an energy replenishment perspective, "to facilitate and cheapen the transmission of intelligence" [9].

2) Background: The current research of SWIPT is still in its infancy, hence there is a paucity of literature. The pioneering

\footnotetext{
${ }^{1}$ See websites: http://www.powercastco.com/.
} 
work of [6] revealed that a non-trivial trade-off exists between energy and capacity for typical channel models, such as the Binary Symmetric Channel (BSC) and the Additive White Gaussian Noise (AWGN) channel, indicating that maximizing the information rate is to a degree coupled with maximizing the energy transfer. When a wideband fading channel is considered, the authors of [8] found that a fundamental trade-off exists between energy and capacity, where the classic water-filling power allocation across the entire frequency band maximizes the attainable information rate, while energy transfer is maximized by transmitting at a single frequency using the total power available, thus reducing the information rate. Similar conclusions were also drawn concerning the fundamental tradeoff for narrowband Multiple Input Multiple Output (MIMO) channels [21], where water-filling based power allocation spanning all eigenvalues of the channel-matrix maximizes the information rate. By contrast, the energy transfer is maximized by concentrating all available power in the specific direction corresponding to the maximum eigenvalue. Further research addressed various other classic channel models, such as the interference channels [22], multiple access channels [23], unicast/multicast channels [24] and secrecy channels [25].

Alongside those theoretical results, practical receiver architectures facilitating SWIPT may be operated in two different modes [21], namely either on a time-division basis or on a power-split basis. To be more specific, in the time-division mode, the receiver alternatively and opportunistically acts as an information detector and power converter [26]. On the other hand, in the power-split mode, the required portion of the received power is used for powering the receiver, while the remaining received power is used for retrieving information [27]. Finally, a hybrid receiver architecture integrating both information and power transfer was proposed in [28]. The respective trade-offs between energy and capacity were characterized for all three receiver architectures of [21], [26]-[28].

In addition to the above one-way SWIPT focusing on receiver architectures, the scope of SWIPT may also be further expanded. For example, the authors of [29], [30] investigated the scenario, where the transmitter conveys power to the receiver, which is then converted to Direct Current (DC) power and reused for the destination's information transmission in the reverse direction. This mode of operation is similar to that of the passive Radio-frequency IDentification (RFID) devices [31] and hence it may be referred to as being two-way half-duplex. More radically, the authors of [32] investigated a two-way fullduplex operational mode, where a pair of nodes interactively communicates and exchanges power. This is also reminiscent of the so-called energy cooperation [33]. Finally, the following set of treatises considered a range of energy-transfer-aided systems, such as multi-carrier systems [34], [35], relay-assisted systems [36] and beamforming-aided systems [37], [38].

3) Novelty: A close inspection of the existing literature reveals that most of the current designs are centred on the energy versus capacity trade-offs, on the receiver architectures and on the attainable system performance under power transfer constraints. It would be however equally beneficial to aim for the full "convergence" of information and power transfer as the ultimate objective in the spirit of Varshney's seminal concept [7], which proposed that energy and information transfer should be innately inter-linked.

Naturally, information is carried by attaching itself to a physical medium, such as radio waves or particles. In optical communications, information is delivered by photons having information-dependent intensities, which may be detected by a photon-counting process [39], [40]. Given the nature of the process, the system is capable of providing a heating/ illumination/propulsion function. Analogously, with the aid of the MIMO concept, we propose a novel technique, where information is carried not by the classic radio waveforms but by energy patterns. More explicitly, our pattern energy embeds information into the specific pattern of the power delivered, which may assume

- a position-based energy pattern, which is reminiscent of the Pulse Position Modulation (PPM) concept, but being invoked in the Spatial Domain (SD).

- an intensity-based energy pattern, which is similar to Pulse Amplitude Modulation (PAM), but exclusively relying on positive values.

Novelty: in this paper, we introduce the energy pattern aided SWIPT concept, where the information is conveyed not only by the Receive Antenna (RA) index of the power delivered but also by the specific intensity of the delivered power. The achievable rate of our technique is immune to any power conversion, thus leading to a beneficial wireless information and power transfer convergence.

The rest of our paper is organised as follows. In Section II, we introduce the underlying concept as well as the transceiver architecture of our energy pattern aided SWIPT system followed by the portrayal of its detection algorithm and its achievable rate analysis. Our simulation results are provided in Section III, while we conclude in Section IV.

\section{SYSTEM DESCRIPTION}

Let us now introduce the underlying concept of energy pattern aided SWIPT as well as its receiver modes, detection algorithm and achievable rate analysis.

\section{A. SWIPT Transmitter}

Consider a MIMO system equipped with $N_{t}$ Transmit Antennas (TA) and $N_{r}$ RA, where we assume $N_{t} \geq N_{r}$. The proposed energy pattern aided SWIPT transmitter relies on the availability of Channel State Information at the Transmitter (CSIT) for supporting Transmitter Pre-Coding (TPC). ${ }^{2}$

1) RA Index-Based Energy Pattern: Our RA index-based energy pattern is generated with the aid of our recently proposed Generalised Pre-coding aided Spatial Modulation (GPSM) scheme [41], [42]. Explicitly, a total of $N_{a}<N_{r}$ RAs are activated, where the particular pattern of the $N_{a}$ RAs activated conveys information in form of so-called SD

\footnotetext{
${ }^{2}$ This scenario has been widely considered in the SWIPT literature [21], [22], [29], [37], [38], since it is desirable to shift most/all signal processing demands from the less powerful receiver that may be passive or semi-passive to the more powerful transmitter that may have access to the mains power.
} 


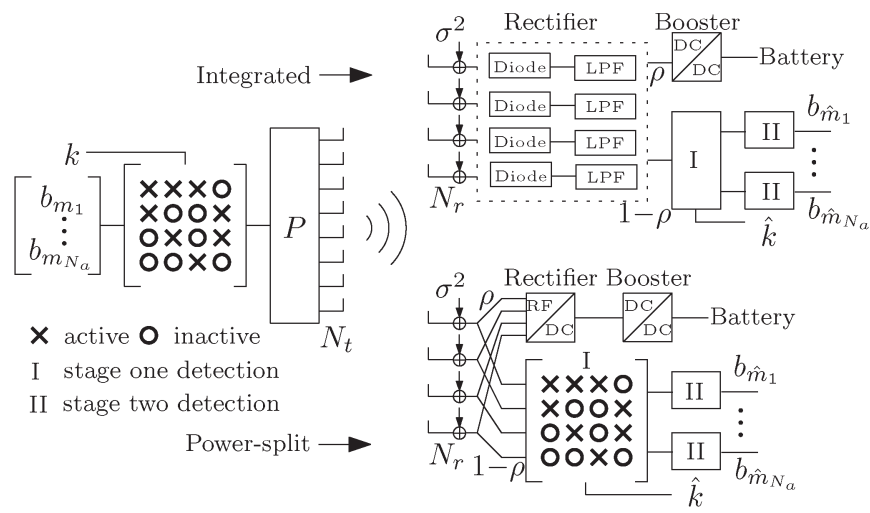

Fig. 1. Transceiver architecture of energy pattern aided SWIPT relying either on an integrated receiver (top) or a power-split receiver (bottom). For example, activating $N_{a}=2$ RAs out of $N_{r}=4$ RAs results in a total of $\left|\mathcal{C}_{t}\right|=6$ legitimate activation patterns, i.e., the patterns of $\mathfrak{C}_{t}=$ $\{[1,2],[1,3],[1,4],[2,3],[2,4],[3,4]\}$. This configuration delivers $k_{p}=2$ bits of information, because $\left\lfloor\log _{2}(|6|)\right\rfloor=2$. Upon selecting for example $\mathcal{C}=$ $\left\{\mathfrak{C}_{t}(1), \mathfrak{C}_{t}(2), \mathfrak{C}_{t}(3), \mathfrak{C}_{t}(4)\right\}$, we have the following mapping between the SD symbol and the activation patterns $k=1 \mapsto \mathfrak{C}(1)=[1,2], k=2 \mapsto \mathfrak{C}(2)=[1,3]$, $k=3 \mapsto \mathcal{C}(5)=[1,4]$, and $k=4 \mapsto \mathcal{C}(6)=[2,3]$.

symbols. Hence, the number of bits conveyed by a SD symbol becomes $k_{p}=\left\lfloor\log _{2}\left(\left|\mathcal{C}_{t}\right|\right)\right\rfloor$, where the set $\mathcal{C}_{t}$ contains all the combinations associated with choosing $N_{a}$ activated RAs out of $N_{r}$ RAs. $^{3}$ To assist our further discussions, let us define the set of selected activation patterns representing a RA index-set as $\mathcal{C} \subset \mathcal{C}_{t}$. We also let $\mathcal{C}(k)$ and $\mathcal{C}(k, i)$ denote the $k$ th $\mathrm{RA}$ activation pattern and the $i$ th activated RA in the $k$ th activation pattern, respectively. An example is given in the caption of Fig. 1.

Let $\boldsymbol{s}_{p}^{k} \in \mathbb{R}_{++}^{N_{r} \times 1}$ be a super-symbol indicating that the RA pattern represented by the index $k$ is activated and $N_{a}$ unit energy pulses $\boldsymbol{b}_{p}=\mathbf{1}^{N_{a} \times 1}$ are delivered. In other words, we have the relationship of

$$
\boldsymbol{s}_{p}^{k}=\Omega_{k} \boldsymbol{b}_{p}
$$

where $\Omega_{k}=\boldsymbol{I}[:, \mathrm{C}(k)]$ is constituted by the specifically selected columns determined by $\mathcal{C}(k)$ of an identity matrix of $\boldsymbol{I}_{N_{r}}$. Following the TPC operation of $\boldsymbol{P} \in \mathbb{C}^{N_{t} \times N_{r}}$, the resultant transmit signal $\boldsymbol{x} \in \mathbb{C}^{N_{t} \times 1}$ may be written as

$$
\boldsymbol{x}=\sqrt{\beta / N_{a}} \boldsymbol{P} \boldsymbol{s}_{p}^{k}
$$

As a natural requirement, the TPC matrix has to ensure that no energy leaks into the RA-elements of the unintended RA patterns. Hence, the classic linear Channel Inversion (CI)-based TPC [43], [44] may be used, which is formulated as

$$
\boldsymbol{P}=\boldsymbol{H}^{H}\left(\boldsymbol{H} \boldsymbol{H}^{H}\right)^{-1}
$$

where $\boldsymbol{H} \in \mathbb{C}^{N_{r} \times N_{t}}$ represents the MIMO channel involved. Furthermore, to avoid any power fluctuation during the preprocessing, we introduce the scaling factor of $\beta$ designed for maintaining the power-constraint of $\mathbb{E}\left[\|x\|^{2}\right]=1$.

\footnotetext{
${ }^{3}$ Note that having a fractional number of bits conveyed by a SD symbol is also possible, but we restrict this number to be an integer in this paper for the ease of implementation and analysis.
}

2) Intensity-Based Energy Pattern: Our intensity-based energy pattern is generated by replacing the above $N_{a}$ unit energy pulses $\boldsymbol{b}_{p}$ with information-bearing energy pulses representing our symbols

$$
\boldsymbol{b}_{m}=\left[b_{m_{1}}, \ldots, b_{m_{N_{a}}}\right]^{T} \in \mathbb{R}_{++}^{N_{a} \times 1}
$$

where we have $b_{m_{i}} \in \mathcal{A}, i \in\left[1, N_{a}\right]$ with its alphabet denoted by $\mathcal{A}$. By dropping the index, let us focus on $b_{m}$, where a total of $k_{s}=\log _{2}(M)$ bits are mapped to one of $M=|\mathcal{A}|$ equi-probable energy pulse based symbols and $\mathbb{E}\left[b_{m}^{2}\right]=1$.

We commence by designing the energy pulse based symbol alphabet relying on Equi-spaced Amplitudes (EA), which are $\mathcal{A}_{E A}=\{1 / d, 2 / d, \ldots, M / d\}$, where we have the energy pulse weights

$$
d=\sqrt{\frac{1+2^{2}+\cdots+M^{2}}{M}}=\sqrt{\frac{(M+1)(2 M+1)}{6}} .
$$

Alternatively, we can design the energy pulse based symbol alphabet relying on Equi-spaced Powers (EP), which are $\mathcal{A}_{E P}=\{1 / d, \sqrt{2} / d, \ldots, \sqrt{M} / d\}$, where we have the energy pulse weights

$$
d=\sqrt{(1+2+\cdots+M) / M}=\sqrt{(M+1) / 2} .
$$

Finally, the optimum design of the alphabet using optimally spaced symbols is left for our future work.

3) Energy Pattern: When combining RA index-based energy pattern and intensity-based energy pattern, (2) becomes

$$
\boldsymbol{x}=\sqrt{\beta / N_{a}} \boldsymbol{P} \boldsymbol{s}_{m}^{k}=\sqrt{\beta / N_{a}} \boldsymbol{P} \boldsymbol{\Omega}_{k} \boldsymbol{b}_{m} .
$$

As a result, the total number of bits transmitted by our energy pattern is $k_{e}=k_{p}+N_{a} k_{s}$. Furthermore, by letting $v_{i}=\mathcal{C}(k, i)$, the scaling factor $\beta$ becomes

$$
\begin{aligned}
\beta & =\frac{N_{a}}{\sum_{i=1}^{N_{a}} \boldsymbol{R}_{v_{i}, v_{i}}^{-1}+\mathbb{E}_{\mathcal{C}}(k)\left[\chi \sum_{i=1}^{N_{a}} \sum_{j=1, j \neq i}^{N_{a}} \boldsymbol{R}_{v_{i}, v_{j}}^{-1}\right]}, \\
& \approx \frac{N_{r}}{\operatorname{Tr}\left[\boldsymbol{R}^{-1}\right]+\chi \sum_{i=1}^{N_{r}} \sum_{j=1, j \neq i}^{N_{r}} \boldsymbol{R}_{i, j}^{-1}}
\end{aligned}
$$

where we have $\boldsymbol{R}=\boldsymbol{H} \boldsymbol{H}^{H}$ and $\chi$ denotes the correlation of the energy pulse based symbols in the intensity-based energy pattern, which is given as

$$
\chi \in\left\{\chi_{E A}, \chi_{E P}\right\}=\left\{\frac{3(M+1)}{4 M+2}, \frac{2\left(\sum_{\mathrm{int}=1}^{M} \sqrt{\mathrm{int}}\right)^{2}}{(M+1)}\right\} .
$$

Finally, the Base Band (BB) signal $x$ is then up-converted to generate the Radio Frequency (RF) signal $x^{R F}$ at a carrier frequency of $f_{c}$ and conveyed through $N_{t}$ TAs.

\section{B. SWIPT Receiver}

The RF signal observed at the $N_{r}$ RAs may be written as

$$
\boldsymbol{r}^{R F}=\boldsymbol{H} \boldsymbol{x}^{R F}+\boldsymbol{w}^{R F}=\sqrt{2} \mathcal{R}\left\{(\boldsymbol{H} \boldsymbol{x}+\boldsymbol{w}) e^{j 2 \pi f_{c} t}\right\}
$$

where $\mathcal{R}\{\cdot\}$ denotes the real operator and $\boldsymbol{w}^{R F}$ is the RF receiver's noise. The equivalent $\mathrm{BB}$ representation of the 
noise $\boldsymbol{w} \in \mathbb{C}^{N_{r} \times 1}$ may be modelled by a circularly symmetric complex-valued Gaussian noise vector with each entry having a zero mean and a variance of $\sigma^{2}$, i.e., we have $\mathbb{E}\left[\|\boldsymbol{w}\|^{2}\right]=\sigma^{2} N_{r}$. Furthermore,

$$
r_{i}^{R F}=\sqrt{2} \mathcal{R}\left\{\left(\boldsymbol{h}_{i} \boldsymbol{x}+w_{i}\right) e^{j 2 \pi f_{c} t}\right\}
$$

represents the RF signal observed at the $i$ th RA and $r_{i}=\boldsymbol{h}_{i} \boldsymbol{x}+w_{i}$ denotes the equivalent BB representation of $r_{i}^{R F}$, where $\boldsymbol{h}_{i}$ is the ith row of $\boldsymbol{H}$.

1) Integrated Model: The integrated receiver concept of Fig. 1 was introduced in [28], where the received RF power is firstly converted to DC power by the rectifiers consisting of a diode and a passive Low-Pass Filter (LPF). Considering (11) and following the analytical models advocated in [15], the current $c_{i}$ output by the non-linear diode for the $i$ th RA is

$$
c_{i}=I_{s}\left(e^{\alpha r_{i}^{R F}}-1\right)=a_{1} r_{i}^{R F}+a_{2}\left(r_{i}^{R F}\right)^{2}+\cdots,
$$

where the coefficients obey $a_{n}=I_{s} \alpha^{n} / n !, n=1,2, \cdots$ with $I_{s}$ being the saturation current and $\alpha$ being the reciprocal of the thermal voltage. Following [28], we reformulate (11)as $r_{i}^{R F}=\sqrt{2}\left|r_{i}\right| \mathcal{R}\left\{e^{2 \pi f_{c} t+\theta}\right\}$ with $\theta=\angle r_{i}$ and insert it into (12), leading to

$$
\begin{aligned}
c_{i} & \approx \sqrt{2} a_{1}\left|r_{i}\right| \mathcal{R}\left\{e^{2 \pi f_{c} t+\theta}\right\}+2 a_{2}\left|r_{i}\right|^{2}\left(\mathcal{R}\left\{e^{2 \pi f_{c} t+\theta}\right\}\right)^{2} \\
& =a_{2}\left|r_{i}\right|^{2}+\sqrt{2} a_{1}\left|r_{i}\right| \mathcal{R}\left\{e^{2 \pi f_{c} t+\theta}\right\}+a_{2}\left|r_{i}\right|^{2} \mathcal{R}\left\{e^{2 \pi 2 f_{c} t+\theta}\right\} .
\end{aligned}
$$

The first approximation of (13) exploits the fact that the impact of higher powers of $r_{i}^{R F}$ is negligible, thus only the first two terms of (12) are considered. Subsequently, the LPF at the output of the diode removes the harmonic components at $f_{c}$ and $2 f_{c}$ of (13), thus we have the DC current of

$$
c_{i}^{0}=\operatorname{LPF}\left(c_{i}\right)=a_{2}\left|r_{i}\right|^{2} .
$$

Note that $a_{2}=I_{s} \alpha^{2} / 2$ is expressed in unit of Amp/Watt and since it is a constant determined by the diode, we assume it to be of unity. ${ }^{4}$ By normalising with respect to $a_{2}$, the DC power harvested is proportional to $\left|r_{i}\right|^{2}$.

After converting the power from RF to DC, the power-splitter of Fig. 1 is employed, resulting into a power transfer path and an information transfer path. For the power transfer path, a portion $\rho$ of the DC power is converted, which is followed by a (multistage) DC to DC booster and energy storage. As a result, the average power transferred by the transmitter and harvested from all the $N_{r}$ RAs is represented as

$$
Q \propto Q=\mathbb{E}\left[\xi \rho \sum_{i=1}^{N_{r}}\left|\tilde{r}_{i}\right|^{2}\right]
$$

where $\tilde{r}_{i}$ denotes the noiseless part of $r_{i}$, representing the power purposely transferred by the transmitter. For the time being, the design of adaptive RA specific power-split ratios $\rho_{i}, i \in\left[1, N_{r}\right]$

\footnotetext{
${ }^{4}$ In [28], the additional noise term of the rectifiers is included and a pulse energy modulation was designed when rectifiers noise is dominant than the antenna noise. However, in this paper, we assume that the RF noise is dominant, where $\boldsymbol{w}$ accounts for both the thermal noise and for the interference.
}

is set aside for our future investigations and we simply set the $\mathrm{RF}$ to DC conversion efficiency to $\xi=0.5$, noting that, it has a non-linear relationship with respect to the incident RF power.

On the other hand, the remaining $1-\rho$ portion of the DC power enters the information transfer path, which may be described for the $i$ th RA as

$$
\mathcal{D}_{i}=(1-\rho)\left|\boldsymbol{h}_{i} \boldsymbol{x}+w_{i}\right|^{2} .
$$

Then the $N_{r}$ detection variables $\left\{\mathcal{D}_{1}, \mathcal{D}_{2}, \cdots, \mathcal{D}_{N_{r}}\right\}$ are used for retrieving the information carried by the energy pattern.

2) Power-Split Mode: The power-split receiver concept of Fig. 1 was introduced in [21], where we have a power transfer path and an information transfer path at the front-end. Explicitly, the received RF signal will be first subject to power conversion at each RA using a power-split ratio of $\rho$. By replacing $r_{i}^{R F}$ in (12) by its power-split version of $\sqrt{\rho} r_{i}^{R F}$ and following the same methodology, the average power transferred by the transmitter and harvested from all the $N_{r}$ RAs is represented the same as for the integrated mode in (15).

After RF to DC power conversion at each RA at a ratio of $\rho$, the remaining RF signal observed at the $i$ th RA may be written as

$$
y_{i}^{R F}=\sqrt{2(1-\rho)} \mathcal{R}\left\{\left(\boldsymbol{h}_{i} \boldsymbol{x}+w_{i}\right) e^{j 2 \pi f_{c} t}\right\}
$$

To retrieve the information carried by the energy pattern, the following $N_{r}$ detection metrics are required $\left\{\left|y_{1}^{R F}\right|^{2},\left|y_{2}^{R F}\right|^{2}, \ldots,\left|y_{N_{r}}^{R F}\right|^{2}\right\}$, where

$$
\left|y_{i}^{R F}\right|^{2}=\left|y_{i}\right|^{2}=(1-\rho)\left|\boldsymbol{h}_{i} \boldsymbol{x}+w_{i}\right|^{2} .
$$

Note that (18) is the same as (16), suggesting that both receiver modes share the same energy detection-like algorithm [45] and hence in the following we will work with (18). We will also show later that $\rho$ has to be set asymptotically close to unity for maximizing the power conversion of (15), since its value does not affect the information transfer based on (18) or (16).

\section{Detection Algorithm}

Following the above discussions, let us focus on the lowcomplexity two-stage detection algorithm by firstly determining the RA indices (SD symbol) of the delivered power and then detecting the intensity (energy pulse based symbol) of the power delivered at that position, while allowing us to defer the optimal joint detection to Section II-E3 owing to its high complexity.

1) Stage-One Detection: The SD symbol may be determined by simply sorting the received power accumulated by each legitimate RA pattern, which is mathematically represented as

$$
\hat{k}=\arg \max _{\ell \in[1,|\mathcal{E}|]}\left\{\sum_{i=1}^{N_{a}}\left|y_{\mathcal{C}(\ell, i)}\right|^{2}\right\} .
$$

Thus, correct hard detection is declared, when we have $\hat{k}=k$. We now formulate the proposition that the power-split ratio $\rho$ does not affect the stage-one detection. 
Proposition II.1: The RA pattern detection of (19) is immune to the power conversion operation of (15) for $\rho<1$.

Proof: As long as we have $c>0$, it can be observed that the decision based on the following metric is equivalent to (19), namely

$$
\hat{k}=\arg \max _{\ell \in[1,|\mathcal{C}|]}\left\{c \sum_{i=1}^{N_{a}}\left|y_{\mathcal{C}(\ell, i)}\right|^{2}\right\} .
$$

Letting $c=1 /(1-\rho)$, we have $\left|y_{\mathcal{C}(\ell, i)}\right|^{2} /(1-\rho)=\left|r_{\mathcal{C}(\ell, i)}\right|^{2}$, which implies that $\rho$ does not affect the decision.

2) Stage-Two Detection: As a subsequent stage after the RA pattern determination, the hard detection of energy pattern intensity invokes the Maximum-Likelihood (ML) criterion, as formulated in Lemma II.1:

Lemma II.1: (Proof in Appendix A) The ML criterion conceived for retrieving the information encapsulated in the intensity-based energy pattern of the $\hat{v}_{i}=\mathcal{C}(\hat{k}, i)$ th RA is

$$
\hat{m}_{i}=\arg \max _{n_{i} \in[1,|\mathcal{A}|]} \mathcal{L}=\arg \max _{n_{i} \in[1,|\mathcal{A}|]} f_{\chi_{2}^{2}}\left(g ; \lambda b_{n_{i}}^{2}\right)_{g=A}
$$

where $\mathcal{L}$ represents the likelihood of the energy pulse based symbol $b_{n_{i}} \in \mathcal{A}$ that results into the observation of $A=\left|r_{\hat{v}_{i}}\right|^{2} / \sigma_{0}^{2}$, where $\sigma_{0}^{2}=\sigma^{2} / 2$. Furthermore, $f_{\chi_{2}^{2}}\left(g ; \lambda b_{n_{i}}^{2}\right)$ represents the Probability Distribution Function (PDF) of the non-central chisquare distribution having two degrees of freedom and a noncentrality of $\lambda b_{n_{i}}^{2}$, where $\lambda=\beta / N_{a} \sigma_{0}^{2}$.

Thus, correct hard detection is declared, when we have $\hat{m}_{i}=$ $m_{i}, i \in\left[1, N_{a}\right]$. We now have the following proposition that the power-split ratio $\rho$ does not affect the stage-two detection.

Proposition II.2: The intensity-based energy pulse symbol detection of (21) is immune to the power conversion operation of (15) for $\rho<1$.

Proof: This is explicit in (21), since the likelihood $\mathcal{L}$ is not a function of the factor $(1-\rho)$. This can be also seen from Appendix A, because the factor $(1-\rho)$ in (35) has been eliminated by normalisation.

\section{Achievable Rate}

Both Proposition II.1 and II.2 suggest that $\rho$ has to be set asymptotically close to unity for maximizing the power conversion of (15), since its value does not affect the information transfer by the operations of (19) and (21), i.e. neither for the power-split receiver nor for the integrated receiver. This implies that for $\rho<1$, the advocated energy pattern aided SWIPT system attains the same information transfer performance. Hence, we proceed by quantifying the achievable rate of the advocated energy patten aided SWIPT system for $\rho<1$.

1) Expression of Achievable Rate: In the previous contributions, Shannon's Continuous-input Continuousoutput Memoryless Channel (CCMC) capacity is quantified under the implicit assumption that the input signal obeys a Gaussian distribution. By contrast, our energy pattern aided SWIPT system is special in the sense that the SD symbols used for RA index-based information transfer convey integer values. Similarly, the energy pulse symbols used for intensity-based information transfer are also in discrete format. Thus the employment of the so-called Discrete-input Continuousoutput Memoryless Channel (DCMC) capacity becomes more appropriate. Since the notion of DCMC capacity implicitly relies on the idealised assumptions of having both an optimal detector and capacity-approaching channel codes, we focus our attention on practical receivers, namely the two-stage detections, where the achievable rate obeying Discrete-input discrete-output Memoryless Channel (DMC) is quantified instead of the DCMC capacity. ${ }^{5}$

More explicitly, we quantify the achievable rate, when employing the two-stage detection algorithm described in Section II-C, where the Mutual Information per Bit (MIB) $I(z ; \hat{z})$ of our energy patten aided SWIPT system measured between the input bits $z \in\{0,1\}$ and the corresponding detected output bits $\hat{z} \in\{0,1\}$ obeys the classic DMC model and it is hence given by:

$$
I(z ; \hat{z})=H(z)-H(z \mid \hat{z})
$$

where $H(z)=-\sum_{z} P_{z} \log _{2} P_{z}$ represents the entropy of the input bits $z$ and $P_{z}$ is the Probability Mass Function (PMF) of $z$. It is noted furthermore that we have $H(z)=1$, when we adopt the common assumption of equal-probability bits, i.e., $P_{z=0}=$ $P_{z=1}=1 / 2$. On the other hand, the conditional entropy $H(z \mid \hat{z})$ represents the average uncertainty concerning $z$ after observing $\hat{z}$, which is given by:

$$
\begin{aligned}
H(z \mid \hat{z}) & =\sum_{\hat{z}} P_{\hat{z}}\left[\sum_{z} P_{z \mid \hat{z}} \log _{2} P_{z \mid \hat{z}}\right] \\
& =-e_{\times} \log e_{\times}-\left(1-e_{\times}\right) \log \left(1-e_{\times}\right)
\end{aligned}
$$

where $e_{\times}$is the crossover probability. By substituting (23) into (22) and exploiting $H(z)=1$ we have:

$$
\begin{aligned}
I(z ; \hat{z}) & =1+e_{\times} \log e_{\times}+\left(1-e_{\times}\right) \log \left(1-e_{\times}\right) \\
& =1+e_{b}^{e} \log e_{b}^{e}+\left(1-e_{b}^{e}\right) \log \left(1-e_{b}^{e}\right)
\end{aligned}
$$

where $e_{b}^{e}$ is the effective Bit Error Ratio (BER) of our energy patten aided SWIPT system representing the transition probability of the DMC. As a result, the achievable rate becomes

$$
R=k_{e} I(z ; \hat{z}) .
$$

2) Expression of Error Probability: Let $e_{b}^{p}\left(e_{s}^{p}\right)$ and $e_{b}^{s}\left(e_{s}^{s}\right)$ represent the BER (Symbol Error Ratio (SER)) of the SD symbol for RA index-based information transfer and of the energy pulse symbol for intensity-based information transfer, respectively. The effective BER may be written as

$$
e_{b}^{e}=\left(k_{p} e_{b}^{p}+N_{a} k_{s} e_{b}^{s}\right) / k_{e} \approx\left(\delta_{k_{p}} e_{s}^{p}+N_{a} e_{s}^{s}\right) / k_{e}
$$

\footnotetext{
${ }^{5}$ Importantly, our achievable rate is calculated for the two-stage detection advocated and this is why it is not referred to as the "capacity". Thus, the achievable rate would naturally become different, when different receivers were to be employed. For example, when soft detection combined with channel coding is employed, the error probability would become lower at low SNRs, leading to a higher achievable rate. When optimal detection combined with idealised capacity-achieving channel coding is employed, the achievable rate would be approaching the DCMC capacity.
} 
where the second half of (26) follows from the approximation of $e_{s}^{s} \approx e_{b}^{s} k_{s}$ as a result of Gray mapping and from the relation $e_{s}^{p} \approx e_{b}^{p} k_{p} / \delta_{k_{p}}$ with the correction factor $\delta_{k_{p}}$ given in Lemma II.2. Thus we find that for the sake of calculating (24) and hence (25), we need the expression of $e_{s}^{p}$ and $e_{s}^{s}$, as formulated in Lemma II.3 and Lemma II.4, respectively.

Lemma II.2: (Proof in Appendix B) The generic expression of the correction factor $\delta_{k_{p}}$ for $k_{p}$ bits is given by:

$$
\delta_{k_{p}}=\delta_{k_{p}-1}+\frac{2^{k_{p}-1}-\delta_{k_{p}-1}}{2^{k_{p}}-1}
$$

where given $\delta_{0}=0$, we can recursively determine $\delta_{k_{p}}$.

Lemma II.3: (Proof in Appendix C) The analytical SER $e_{s}^{p}$ of the SD symbol for RA index-based information transfer may be upper bounded by $e_{s, u b}^{p}$ as:

$$
e_{s}^{p}<1-\mathbb{E}_{\boldsymbol{b}_{m}}\left[\int_{0}^{\infty} \bar{e}_{s, l b}^{p}\left(\lambda, \boldsymbol{b}_{m}\right) f_{\lambda}(\lambda) d \lambda\right]=e_{s, u b}^{p}
$$

where we have $\lambda=\beta / N_{a} \sigma_{0}^{2}$ and the lower bound of the probability of correct detection of the SD symbol when the energy pattern based symbol $\boldsymbol{b}_{m}$ was transmitted becomes

$$
\bar{e}_{s, l b}^{p}\left(\lambda, \boldsymbol{b}_{m}\right)=\prod_{i=1}^{N_{a}} \int_{0}^{\infty}\left[F_{\chi_{2}^{2}}(g)\right]^{N_{r}-N_{a}} f_{\chi_{2}^{2}}\left(g ; \lambda b_{m_{i}}^{2}\right) d g
$$

where $F_{\chi_{2}^{2}}(g)$ represents the Cumulative Distribution Function (CDF) of a chi-square distribution having two degrees of freedom, while $f_{\chi_{2}^{2}}\left(g ; \lambda b_{m_{i}}^{2}\right)$ represents the PDF of a non-central chi-square distribution having two degrees of freedom and noncentrality given by $\lambda b_{m_{i}}^{2}$ with the PDF of $f_{\lambda}(\lambda)$.

Lemm II.4: (Proof in Appendix D) The analytical SER $e_{s}^{s}$ of the energy pulse based symbols for intensity-based information transfer may be upper bounded by $e_{s, u b}^{s}$ as:

$$
e_{s}^{s}<\left(1-e_{s, u b}^{p}\right) \tilde{e}_{s, u b}^{s}+e_{s, u b}^{p} \frac{\sum_{\ell \neq k} N_{c} \tilde{e}_{s, u b}^{s}+N_{d} e_{s}^{o}}{N_{a}\left(2^{k_{p}}-1\right)}=e_{s, u b}^{s}
$$

where $N_{c}$ and $N_{d}=\left(N_{a}-N_{c}\right)$ represent the number of common and different RA between $\mathrm{C}(\ell)$ and $\mathrm{C}(k)$, respectively. Furthermore, $e_{s}^{o}=(M-1) / M$ and $\tilde{e}_{s, u b}^{s}$ denotes the SER upper bound of the energy pulse based symbols in the absence of SD symbol errors, which is given by (31), where $\lambda=\beta / N_{a} \sigma_{0}^{2}, b_{m} \in \mathcal{A}$ and $t_{m}$ as seen in Appendix D.

$$
\begin{aligned}
\tilde{e}_{s, u b}^{s}=\frac{1}{M} \int_{0}^{\infty}[ & 1-F_{\chi_{2}^{2}}\left(g ; \lambda b_{1}^{2}\right)_{g=t_{1}} \\
& +F_{\chi_{2}^{2}}\left(g ; \lambda b_{M}^{2}\right)_{g=t_{M-1}} \\
& +\sum_{m=2}^{M-1} F_{\chi_{2}^{2}}\left(g ; \lambda b_{m}^{2}\right)_{g=t_{m-1}} \\
& \left.+1-F_{\chi_{2}^{2}}\left(g ; \lambda b_{m}^{2}\right)_{g=t_{m}}\right] f_{\lambda}(\lambda) d \lambda .
\end{aligned}
$$

Finally, we get the upper bound of the effective BER by substituting the (28) and (30) into (26). This is then used to get the lower bound of (24) and of (25), respectively, where we use the simplified notation of $I$ and $R$, instead of $I^{l b}$ and $R^{l b}$.

\section{E. Further Discussions}

Let us finally address some further issues concerning the energy pattern aided SWIPT system advocated.

1) Derivatives: Let us for a moment consider only the RA index-based energy pattern, where unit-energy pulses $\boldsymbol{b}_{p}$ are used in conjunction with $\chi=1$ in (8). Hence, using the stageone detection of (19) is sufficient. Another potential systemconfiguration is when only the intensity-based energy pattern detection of (21) is invoked in conjunction with $N_{a}=N_{r}$.

When the information is embedded in the TA indices, rather than in the RA indices, we arrive at the so-called Spatial Modulation (SM) concept [46]. However, SM may not be used in a SWIPT system relying on an integrated receiver, because Zero-Forcing (ZF) would be required at the receiver for determining the TA indices. This BB operation cannot be performed on the energy metrics of (16) harvested at the output of rectifiers and this holds also true for ML-based SM receivers. Similarly, the ingenious Sub-carrier Index Modulation (SIM) of [47] achieved by embedding information in sub-carrier index patterns may also be unsuitable for SWIPT systems relying on an integrated receiver because of the BB Fast Fourier Transform (FFT) operation.

2) Alternatives: Without using the CI-based TPC, we may design an alternative energy pattern aided SWIPT system by employing a Maximum Eigenvalue (ME) aided TPC. In the ME-TPC aided arrangement, only a single information stream is precoded using the right-singular vector $\boldsymbol{\mu}_{r}^{*}$ corresponding to the maximum eigenvalue $\lambda_{e}^{*}$ of the MIMO channel $\boldsymbol{H}$. However, the challenge is that the left-singular vector $\boldsymbol{\mu}_{l}^{*}$ corresponding to $\lambda_{e}^{*}$ of the MIMO channel $\boldsymbol{H}$ used for post-processing at the receiver is also required for intensity-based information transfer. Although this post-processing is typically applied at $\mathrm{BB}$, based on the solutions developed in [48], [49], it may also be realised at the RF front-end [50], [51]. ${ }^{6}$ Thus, in the ME-TPC aided arrangement, first post-processing is applied at $\mathrm{RF}$ and as a result, the associated power conversion becomes capable of harvesting $\mathcal{Q} \propto Q=\mathbb{E}\left[\xi \rho \lambda_{e}^{*} b_{m}^{2}\right]$ amount of power. By assigning all the available power to the direction corresponding to the maximum eigenvalue, the amount of transferred power is thus maximised at the cost of a reduced information rate, since only a single information stream is supported as detailed in [21]. As for the information transfer, the metric $\left|\lambda_{e}^{*} b_{m}+\boldsymbol{\mu}_{l}^{*} \boldsymbol{w}\right|^{2}$ is formed, which is then maximized as seen in (21).

As an important practical aspect, let us now consider the channel knowledge requirement of our energy pattern aided SWIPT system. For our CI-TPC aided arrangement, the stageone detection of (19) is entirely non-coherent, hence no channel knowledge is required at the receiver. By contrast, the stagetwo detection of (21) needs the estimates of $\beta$, which may be approximated according to [52]. Hence, we may assume a Time Division Duplex (TDD) operation for the acquisition of CSIT, where the receiver sends its uplink pilot sequences to the transmitter, which are used for estimating the uplink channel.

\footnotetext{
${ }^{6}$ The beamforming solutions based on SVD plus waterfilling and its variants are excluded here, since they rely on post-multiplication by the conjugate of the left unitary matrix of the channel's SVD, which can only be realised in the baseband.
} 
The resultant uplink channel information is then calibrated and reused for the downlink SWIPT, which assumes reciprocity between the uplink and downlink channels in the TDD mode. On the other hand, in the ME-TPC aided arrangement, both the transmitter and the receiver requires the availability of channel knowledge, thus it is more complex and less practical than the CI-TPC aided arrangement.

3) Complications: We now discuss the optimal joint detection of both the intensity-based energy pattern and of the SD symbol $k$. By defining $\mathcal{D}=\left\{\left|y_{1}\right|^{2}, \cdots,\left|y_{N_{r}}\right|^{2}\right\}$, the ML criterion based optimal joint detection is formulated as

$$
\left[\hat{m}_{1}, \ldots, \hat{m}_{N_{a}}, \hat{k}\right]=\underset{\boldsymbol{s}_{n}^{\ell} \in \mathcal{B}}{\arg \max } p\left(\mathcal{D} \mid \boldsymbol{s}_{n}^{\ell}\right)
$$

where $\mathcal{B}=\mathcal{C} \times \mathcal{A}^{N_{a}}$ is the joint search space of the supersymbol $\boldsymbol{s}_{n}^{\ell}$, which may be explicitly expanded as $\mathcal{B}=\{\ell \in$ $\left.[1,|\mathcal{C}|], n_{i} \in[1,|\mathcal{A}|], i \in\left[1, N_{a}\right]\right\}$. More explicitly, the likelihood function $\mathcal{L}_{n}^{\ell}=p\left(\mathcal{D} \mid \boldsymbol{s}_{n}^{\ell}\right)$ is given as

$$
\mathcal{L}_{n}^{\ell}=\prod_{i=1}^{N_{a}} f_{\chi_{2}^{2}}\left(\frac{\left|r_{\mathcal{C}(\ell, i)}\right|^{2}}{\sigma_{0}^{2}} ; \lambda_{n_{i}}^{2}\right) \prod_{j=1}^{N_{a}^{\prime}} f_{\chi_{2}^{2}}\left(\frac{\left|r_{\overline{\mathcal{C}}(\ell, j)}\right|^{2}}{\sigma_{0}^{2}}\right)
$$

where we have $N_{a}^{\prime}=N_{r}-N_{a}, \lambda=\beta / N_{a} \sigma_{0}^{2}$ and $\sigma_{0}^{2}=\sigma^{2} / 2$. Since the expansion of (33) becomes trivial with the aid of our proofs of Lemma II.1 and II.3 in Appendix A and C, we omit it for the sake of space economy.

It is thus clear that the two-stage detection algorithm advocated in Section II-C constitutes a low-complexity alternative to the joint detection of (32). More explicitly, the two-stage detection represents a sequential low-complexity implementation of the joint detection, where the stage-one detection finds the RA pattern with respect to arbitrary $\boldsymbol{b}_{n}$, while the stagetwo detection finds the most likely $\boldsymbol{b}_{n}$ given the estimate of the SD symbol $\hat{k}$ provided by the stage-one detection. It is also clear that the joint detection has a complexity on the order of $|\mathcal{C}| \times|\mathcal{A}|^{N_{a}}$, while our two-stage detection has a complexity on the order of $|\mathcal{C}|+N_{a}|\mathcal{A}|$.

Proposition II.3: The stage-one SD symbol detection of (19) obeys the ML criterion.

Proof: In the stage-one detection of Section II-C1, given an arbitrary $\boldsymbol{b}_{n} \in \mathcal{A}^{N_{a}}$, the optimal ML criterion for determining the $\mathrm{SD}$ symbol $\hat{k}$ is formulated as

$$
\begin{aligned}
& \hat{k}=\arg \max _{\ell \in[1,|\mathcal{E}|]} p\left(\mathcal{D} \mid \Omega_{\ell}, \boldsymbol{b}_{n}\right)=\arg \max _{\ell \in[1,|\mathcal{E}|]} \mathcal{L}_{n}^{\ell} \\
& \stackrel{a}{a} \arg \max _{\ell \in[1,|\mathcal{E}|]} \prod_{i=1}^{N_{a}} I_{0}\left(\sqrt{\frac{\lambda b_{n_{i}}^{2}}{(1-\rho) \sigma_{0}^{2}}\left|y_{\mathcal{C}(\ell, i)}\right|^{2}}\right) \\
& \stackrel{b}{\propto \arg } \max _{\ell \in[1,|\mathcal{E}|]}\left\{\prod_{i=1}^{N_{a}}\left|y_{\mathcal{C}(\ell, i)}\right|^{2}\right\} \stackrel{c}{\propto} \underset{\arg }{\max }\left\{\prod_{\ell \in[1,|\mathcal{E}|]}^{N_{a}} e_{i=1}^{\left|y_{\mathcal{E}(\ell, i)}\right|^{2}}\right\} \\
& \propto \arg \max _{\ell \in[1,|\mathcal{C}|]}\left\{\sum_{i=1}^{N_{a}}\left|y_{\mathcal{C}(\ell, i)}\right|^{2}\right\}
\end{aligned}
$$

where in (34-a) we omitted the irrelevant terms after expanding (33). Furthermore, in (34-b) and (34-c), we exploited the fact that the modified zeroth-order first kind Bessel function $I_{0}(\cdot)$ and the exponential function are both monotonically increasing positive convex function.

\section{Performance Evaluation}

Let us now characterize the performance of our energy pattern aided SWIPT system advocated.

\section{A. Configuration}

In our simulations, the transmit power is set to $P_{t}=30 \mathrm{dBm}$ or 1 watt and the path-loss model obeys $P_{r}=P_{t} A_{t} A_{r}\left(d_{0} \lambda_{c}\right)^{-\alpha_{p l}}$, where $d_{0}$ is the distance between TA and RA array, $\lambda_{c}$ denotes the wavelength corresponding to a carrier frequency of 5.8 $\mathrm{GHz}$, the path-loss exponent is set to $\alpha_{p l}=4$ and the aperture of the TA and RA is represented by $A_{t}$ and $A_{r}$, respectively with the per-antenna aperture being set to $1 \mathrm{~cm}^{2}$. Each entry of the channel-matrix $\boldsymbol{H}$ undergoes frequency-flat Rician fading and it is uncorrelated between the different super-symbol instants, but remains constant within the duration of a supersymbol. When modelling the Line Of Sight (LOS) path of the Rician channel, we employ the far-field uniform linear antenna array at both the transmitter and receiver [53], with their responses represented as $\left[1, e^{-j \pi / 2}, \cdots, e^{-j \pi\left(N_{t}-1\right) / 2}\right]$ and as $\left[1, e^{-j \pi / 2}, \cdots, e^{-j \pi\left(N_{r}-1\right) / 2}\right]$, respectively. We assume having perfect channel information at both the receiver as well as at the transmitter and set aside the effects of CSIT-errors for our future investigations [37], [41]. Finally, the RF antenna induced noise $w$ accounts for both the thermal noise as well as for the interference and it is thus set to $\sigma^{2}=-50 \mathrm{dBm}$. This assumption is valid as for the non-licence band at $5.8 \mathrm{GHz}$ considered, interference could be pervasive. Also, the RF to DC conversion efficiency is set to $\xi=0.5$.

\section{B. Preparatory Results}

1) BER Performance: Fig. 2 shows both the analytical and simulated performance of a $\left\{N_{t}, N_{r}\right\}=\{8,4\}$ energy pattern aided SWIPT system, characterizing its effective BER (left) for $M$-ary intensity-based energy pattern using the equi-spaced amplitudes $\mathcal{A}_{E A}$ and experiencing Rayleigh fading with $\rho<1$. In addition, we also characterize the BER relying on the RA index-based energy pattern in isolation (right), namely the BER as a result of pure stage-one detection. It is clear that our analytical results form a tight upper bound of the simulated results in both subplots of Fig. 2. As expected, when the RA's distance from the TAs increases, the BER performance degrades in both subfigures of Fig. 2. Furthermore, as seen in the left subplot of Fig. 2, the higher the ratio of $N_{a} / N_{t}$, the worse the BER performance becomes. Similarly, observe in the right subplot of Fig. 2 that a higher number of activated RA leads to a degraded BER performance, since the receiver becomes more prone to decision errors.

2) Information Transfer: Fig. 3 shows the achievable rate of a $\left\{N_{t}, N_{r}\right\}=\{8,4\}$ energy pattern aided SWIPT system employing various $M$-ary intensity-based energy pattern using the equi-spaced amplitudes $\mathcal{A}_{E A}$ and experiencing Rayleigh fading

\footnotetext{
${ }^{7}$ Practically, the RF to DC conversion efficiency has a non-linear relationship with respect to the incident RF power, which is closely related to the particular design of the power conversion circuits. Setting it to 0.5 constitutes a realistic choice, since the true value reported at http://www.powercastco.com/ states that it can be higher or lower.
} 

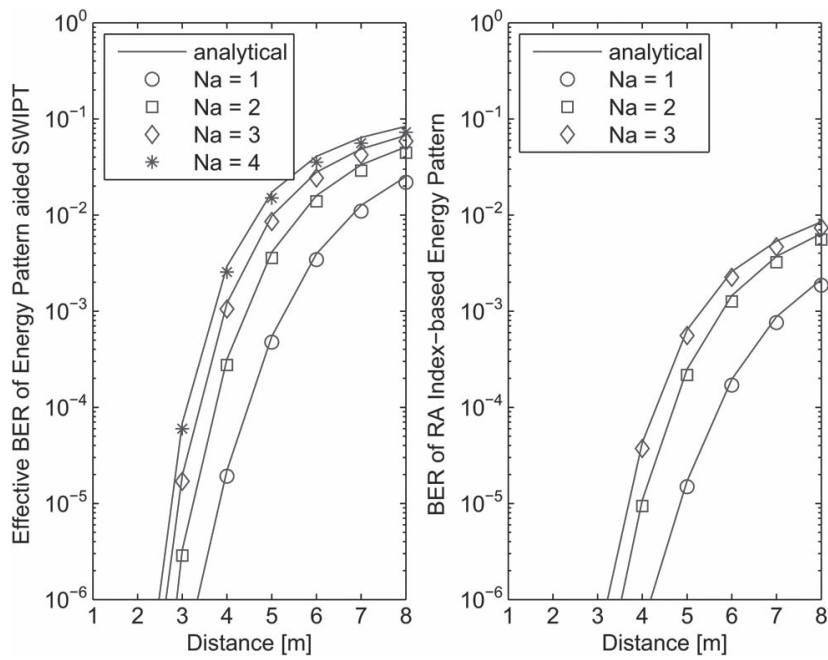

Fig. 2. Analytical and simulated performance of a $\left\{N_{t}, N_{r}\right\}=\{8,4\}$ energy pattern aided SWIPT system, characterizing both the effective BER of the energy pattern (left) and the BER of the RA index-based energy pattern in isolation (right). In addition, we assume $M$-ary intensity-based energy pattern using the equi-spaced amplitudes $\mathcal{A}_{E A}$ and experiencing Rayleigh fading with $\rho<1$.
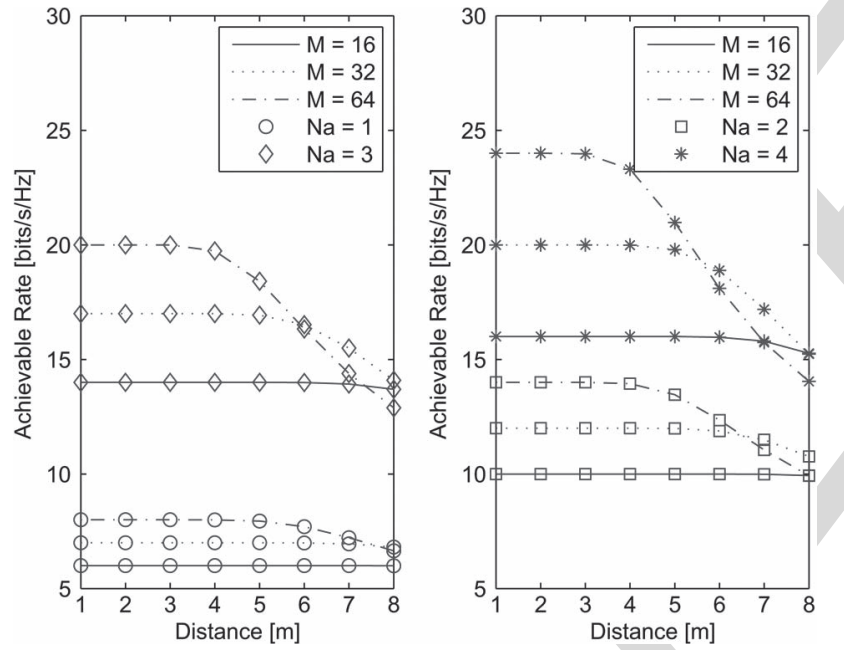

Fig. 3. Achievable rate of a $\left\{N_{t}, N_{r}\right\}=\{8,4\}$ energy pattern aided SWIPT system employing various $M$-ary intensity-based energy pattern using the equispaced amplitudes $\mathcal{A}_{E A}$ and experiencing Rayleigh fading with $\rho<1$.

with $\rho<1$. It may be seen from Fig. 3 that the higher the value of $N_{a} / N_{t}$, the higher the attainable rate becomes. Importantly, by employing RA index-based information transfer, extra SD symbols are also conveyed. When investigating a fixed $N_{a}$, $M$-ary energy pulse based symbols are preferred from an achievable rate perspective, when the receiver is sufficiently close to the transmitter. Naturally, $M$ has to be gradually reduced, when the receiver is further apart from the TA. This suggests that a distance-dependent adaptive energy pattern regime may be conceived for our future work.

3) Power Transfer: Fig. 4 shows the power conversion of an $\left\{N_{t}, N_{r}\right\}=\{8,4\}$ (left) and of an $\left\{N_{t}, N_{r}\right\}=\{4,2\}$ (right) energy pattern aided SWIPT system employing $M$-ary intensitybased energy patterns using the equi-spaced amplitudes $\mathcal{A}_{E A}$ and experiencing Rician fading associated with $K=[0,1,3,5]$ and $\rho=1$, where both the CI-TPC aided arrangement and
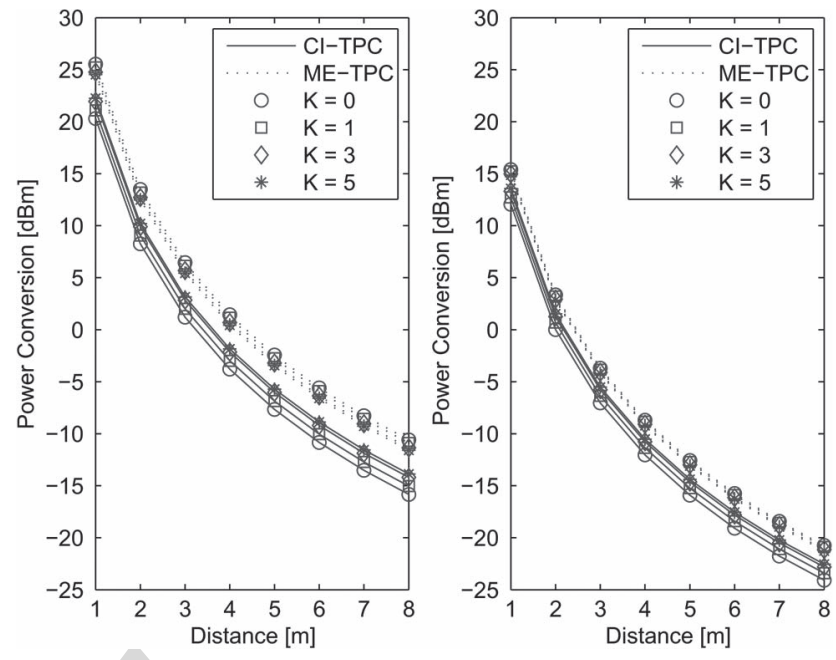

Fig. 4. Power conversion of a $\left\{N_{t}, N_{r}\right\}=\{8,4\}$ (left) and $\left\{N_{t}, N_{r}\right\}=\{16,8\}$ (right) energy pattern aided SWIPT system experiencing employing $M$-ary intensity-based energy patterns using the equi-spaced amplitudes $\mathcal{A}_{E A}$ and experiencing Rician fading associated with $K=[0,1,3,5]$ and $\rho=1$, where both the CI-TPC aided arrangement and the ME-TPC aided arrangement are included.

the ME-TPC aided arrangement are included, since the latter is capable of maximising the attainable power conversion. Observe by comparing the left and right subplot of Fig. 3 that the higher the number of TAs and RAs, the higher the converted power and the higher the performance-discrepancy between the CI-TPC and the ME-TPC aided arrangements.

Another interesting finding is that the higher the Rician $K$-factor, the higher the converted power of the CI-TPC aided arrangement. This is because when CI is used for our energy pattern aided SWIPT system, the reduced power conversion experienced is due to the detrimental effect of CI, when the channel is more dramatically faded. Thus, a high value of $K$ implies more benign LOS propagation, leading to an increased power conversion. On the other hand, the higher the value of $K$, the lower the converted power for the ME-TPC aided arrangement. This is because a higher value of $K$ leads to a reduced ratio between the maximum and minimum eigenvalues. Finally, the performance-dependence of the CI-TPC aided arrangement on $K$ is more obvious than that of the ME-TPC aided arrangement.

\section{Trade-Offs Results}

1) Trade-Offs: Fig. 5 shows the trade-offs between the achievable rate as well as power conversion of a $\left\{N_{t}, N_{r}\right\}=$ $\{8,4\}$ energy pattern aided SWIPT system employing $M$-ary intensity-based energy patterns using the equi-spaced amplitudes $\mathcal{A}_{E A}$ and experiencing Rician fading associated with $K=$ $[0,1,3,5]$ at $d_{0}=5 \mathrm{~m}$, where both the CI-TPC aided arrangement and the ME-TPC aided arrangement are characterised.

Let us now investigate the so-called trade-off bound for each $N_{a}$, which consists of a horizontal line segment, a sloping diagonal line segment and a vertical line segment. Firstly, since Proposition II.1 and II.2 implies that the power conversion of the CI-TPC aided arrangement does not affect the achievable rate, this is explicitly illustrated by the horizontal line segment 


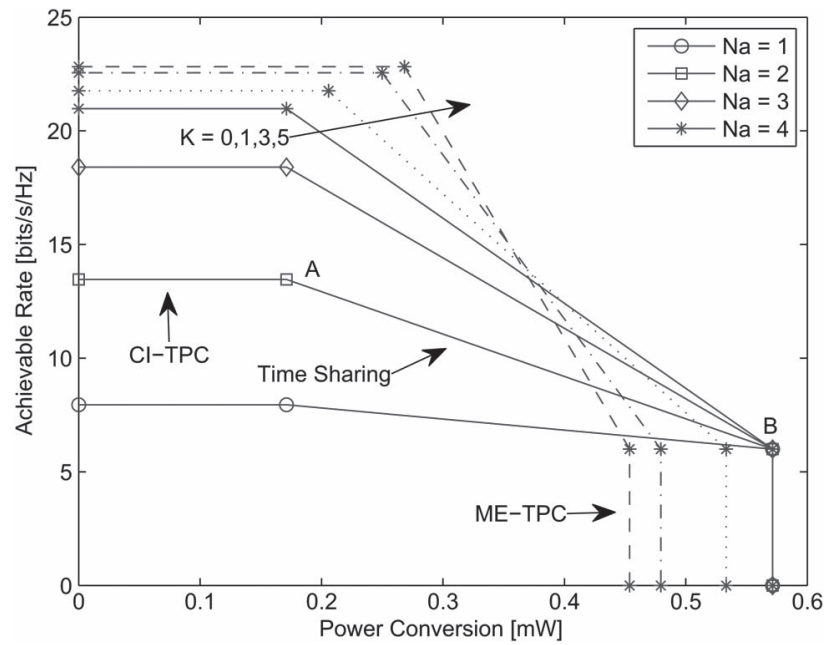

Fig. 5. Trade-offs between the achievable rate and power conversion of a $\left\{N_{t}, N_{r}\right\}=\{8,4\}$ energy pattern aided SWIPT system employing $M$-ary intensity-based energy patterns using the equi-spaced amplitudes $\mathcal{A}_{E A}$ and experiencing Rician fading associated with $K=[0,1,3,5]$ at $d_{0}=5 \mathrm{~m}$, where both the CI-TPC aided arrangement and the ME-TPC aided arrangement are characterised.

associated with all the legends corresponding to different $N_{a}$ values. This implies that if the required power conversion is less than A, as shown in Fig. 5, then employing the CI-TPC aided arrangement would result in a high achievable rate. On the other hand, when ME-TPC aided arrangement is considered, the maximum power conversion may be achieved in conjunction with a rate of $6 \mathrm{bits} / \mathrm{s} / \mathrm{Hz}$, since only a single intensity-based information stream is allowed. This is illustrated by the vertical line segment, implying that when the achievable rate is lower than B, as shown in Fig. 5, then employing the ME-TPC aided arrangement would achieve a high power conversion. Finally, the remaining segment of the trade-off bound may be characterised by the sloping diagonal line segment connecting the bounds of the CI-TPC aided arrangement and the METPC aided arrangement, where a time-sharing strategy may be employed, as indicated in Fig. 5.

Two further observations emerge from the Fig. 5. Firstly, the higher the ratio $N_{a} / N_{r}$, the larger the area under the trade-off bound. Secondly, as $K$ increases, having a less severely faded channel leads to a higher achievable rate and a higher power conversion. Thus we observe an enlarged trend of the trade-off bound towards the CI-TPC aided arrangement. By contrast, we have a shrinking trend of the trade-off bound towards the METPC aided arrangement. This is because a higher value of $K$ leads to a reduced ratio between the maximum and minimum eigenvalues.

2) Comparisons: The left subplot of Fig. 6 shows the tradeoffs between the goodput and power conversion of a $\left\{N_{t}, N_{r}\right\}=$ $\{8,4\}$ energy pattern aided SWIPT system employing $M$-ary intensity-based energy patterns using the equi-spaced amplitudes $\mathcal{A}_{E A}$ and experiencing Rayleigh fading at $d_{0}=5 \mathrm{~m}$, where both the CI-TPC aided arrangement and the ME-TPC aided arrangement are characterised. Using the achievable rate as our metric as in Fig. 5 partially conceals the fact that the MIB for a low value of $N_{a}$ is actually higher than that of a high $N_{a}$, as demonstrated by the BER results in the right subplot of Fig. 2 .
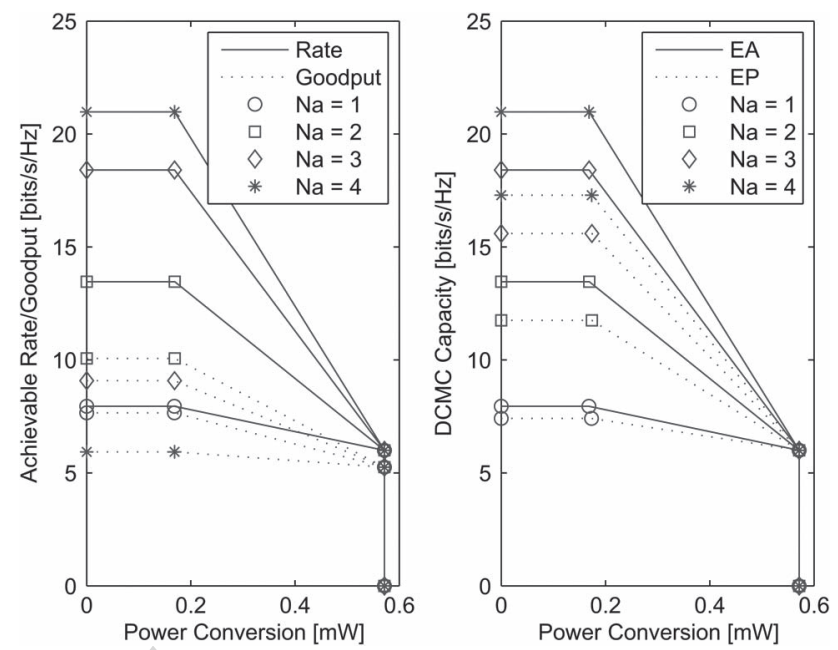

Fig. 6. Trade-off between the goodput and power conversion (right) and between the achievable rate and power conversion when $\mathcal{A}_{E P}$ is used instead of $\mathcal{A}_{E A}$. In both subplots, a $\left\{N_{t}, N_{r}\right\}=\{8,4\}$ energy pattern aided SWIPT system is considered, where both the CI-TPC aided arrangement and the METPC aided arrangement are characterised for an $M$-ary intensity-based energy pattern at $d_{0}=5 \mathrm{~m}$ and experiencing Rayleigh fading.

Thus, apart from the achievable rate, the reliability of each transmitted bit is also important. We defined the goodput as $1-\left(1-e_{b}^{e}\right)^{L}$, where $L$ represents the frame length, which is set to $L=100$. This may also be referred to as the BLock Error Ratio (BLER) indicating the Quality of Service (QoS), where we may observe that in contrast to using the achievable rate as our metric, it is no longer true that a higher $N_{a} / N_{r}$ value leads to a larger area under the trade-off bound.

The right subplot of Fig. 6 characterizes the trade-off between the achievable rate and the power conversion of a $\left\{N_{t}, N_{r}\right\}=\{8,4\}$ SWIPT system employing $M$-ary intensitybased energy patterns using either the equi-spaced amplitudes $\mathcal{A}_{E A}$ or the equi-spaced power $\mathcal{A}_{E P}$ and experiencing Rayleigh fading at $d_{0}=5 \mathrm{~m}$, where both the CI-TPC aided arrangement and the ME-TPC aided arrangement are characterised. It is clearly shown in the right subplot of Fig. 6 that the EA design of intensity-based energy pattern exhibits a higher achievable rate, while maintaining the same level of power conversion as the EP design. However, this is achieved at the cost of exhibiting a higher Peak to Average Power Ratio (PAPR).

\section{CONCLUSION}

A novel energy pattern aided SWIPT system was proposed, where both RA index-based information transfer as well as intensity-based information transfer were employed in the context of both the integrated receiver mode and the power-split mode of Fig. 1, where two-stage detection algorithms were used. The analytical BER of both the SD symbols, as well as of the energy pulse based symbols and of the overall energy pattern were also derived. Both the analytical and simulation results demonstrate that our energy pattern aided SWIPT system exhibits a beneficial immunity to power conversion. Our proposed energy pattern based SWIPT system leads to a beneficial wireless information and power transfer convergence, where the achievable rate versus power conversion trade-off bounds 
and the goodput versus power conversion trade-off bounds were also discussed. Hence, the energy pattern aided SWIPT system may be viewed as an instantiation of joint information and energy transfer in the spirit of Varshney's seminal concept [7], where information and energy transfer are inter-linked.

In fact, our conclusions concerning the immunity to power conversion revealed in our propositions are idealized. Practically, $\rho$ cannot be tuned to be asymptotically close to unity, since a certain minimum required power would be reserved for information transfer, where the amount of reserved power depends on the sensitivity of the practical detection circuit. Moreover, the information transfer performance would not be independent of $\rho$ owing to the finite processing accuracy of the practical detection circuit, which exhibits its own imperfections and limitations as a function of the incident power. Hence, a promising future research topic would be to exploit the flexibility of our design by adapting the choice of $N_{a}$ and by balancing it along the trade-off curves with the above-mentioned practical aspects explicitly considered.

\section{APPENDIX A}

\section{PROOF OF LEMMA II.1}

Let us assume that the first-stage detection of (19) suggested that the RA pattern $\hat{k}$ was activated. Then, upon considering the $\hat{v}_{i}=\mathcal{C}(\hat{k}, i)$ th activated RA, the ML detector determines the most likely energy pulse based symbols $b_{n_{i}} \in \mathcal{A}$ that results into the observation of $\left|y_{\hat{v}}\right|^{2}$ in (18), when we have equi-probable $b_{n_{i}}$ values.

Assuming that $b_{n_{i}}$ was transmitted, we have the observation given by

$$
\begin{aligned}
G & =(1-\rho)\left|\sqrt{\beta / N_{a}} b_{n_{i}}+w_{\hat{v}_{i}}\right|^{2}, \\
& =(1-\rho)\left[\left(\sqrt{\beta / N_{a}} b_{n_{i}}+\mathcal{R}\left\{w_{\hat{v}_{i}}\right\}\right)^{2}+\mathcal{J}\left\{w_{\hat{v}_{i}}\right\}^{2}\right] .
\end{aligned}
$$

By normalising (35) with respect to $(1-\rho) \sigma_{0}^{2}$, it is readily seen that $g=G /(1-\rho) \sigma_{0}^{2}$ obeys

$$
g \sim\left[\mathcal{N}\left(\sqrt{\lambda} b_{n_{i}}, 1\right)\right]^{2}+[\mathcal{N}(0,1)]^{2}=\chi_{2}^{2}\left(g ; \lambda b_{n_{i}}^{2}\right)
$$

where $\lambda=\beta / N_{a} \sigma_{0}^{2}$. Thus, it is clear that the likelihood of $b_{n_{i}}$ that results into the observation of $A=\left|y_{\hat{v}_{i}}\right|^{2} /(1-\rho) \sigma_{0}^{2}=$ $\left|r_{\hat{v}_{i}}\right|^{2} / \sigma_{0}^{2}$ is

$$
\mathcal{L}=f_{\chi_{2}^{2}}\left(g ; \lambda b_{n_{i}}^{2}\right)_{g=A} .
$$

As a result, the ML detection criterion is given by (21).

\section{APPENDIX B \\ PROOF OF LEMMA II.2}

Let $\mathcal{A}_{k_{p}}$ denote the alphabet of the SD symbol having $k_{p}$ bits of information. Then the cardinality of the alphabet $\mathcal{A}_{k_{p}}$ is twice higher compared to that of $\mathcal{A}_{k_{p}-1}$. Thus, $\mathcal{A}_{k_{p}}$ may be constructed by a pair of sub-alphabets of $\mathcal{A}_{k_{p}-1}$, represented by 0 and 1, respectively. We may thereafter refer to the alphabet of $\mathcal{A}_{k_{p}-1}$ preceded by the above-mentioned 0 as the zero-alphabet, while the other one as the one-alphabet.
Assuming that the SD symbol representing $k_{p}$ zeros was transmitted, we may then calculate the total number of pairwise bit errors $\varepsilon_{0}$ in the above-mentioned zero-alphabet. Hence, the number of pair-wise bit errors $\varepsilon_{1}$ in the one-alphabet is simply $\varepsilon_{1}=\varepsilon_{0}+2^{k_{p}}$, which accounts for the difference in the first preceding bit. Hence the total number of pair-wise bit errors is $\varepsilon=2 \varepsilon_{0}+2^{k_{p}}$. Assuming an equal probability of $1 /\left(2^{k_{p}}-1\right)$ for each possible SD symbol error, we arrive at the correction factor given by $\delta_{k_{p}}=\left(2 \varepsilon_{0}+2^{k_{p}}\right) /\left(2^{k_{p}}-1\right)$.

Since $\varepsilon_{0}$ represents the total number of pair-wise bit errors corresponding to the case of $\left(k_{p}-1\right)$ bits of information, we have $\varepsilon_{0}=\left(2^{k_{p}-1}-1\right) \delta_{k_{p}-1}$. Hence the resultant expression of the correction factor may be calculated recursively according to (27) after some further manipulations.

\section{APPENDIX C}

\section{PROOF OF LEMMA II. 3}

Considering the general case of $N_{r}$ as well as $N_{a}$ and assuming that the RA pattern $\mathcal{C}(k)$ was activated, according to Proposition II.1 and dropping $\rho$, for each metric of (18), we have for $v_{i} \in \mathcal{C}(k)$ and $u_{j} \in \overline{\mathcal{C}}(k)$ :

$$
r_{v_{i}}=\sqrt{\beta / N_{a}} b_{m_{i}}+w_{v_{i}} ; \quad r_{u_{j}}=w_{u_{j}}
$$

where $\overline{\mathcal{C}}(k)$ denotes the complementary set of the activated RA pattern $\mathcal{C}(k)$ in $\mathcal{C}$. Upon introducing $\sigma_{0}^{2}=\sigma^{2} / 2$, we have:

$$
\begin{aligned}
\left|r_{y_{i}}\right|^{2} & \sim\left[\mathcal{N}\left(\sqrt{\beta / N_{a}} b_{m_{i}}, \sigma_{0}^{2}\right)\right]^{2}+\left[\mathcal{N}\left(0, \sigma_{0}^{2}\right)\right]^{2} \\
\left|r_{u_{j}}\right|^{2} & \sim\left[\mathcal{N}\left(0, \sigma_{0}^{2}\right)\right]^{2}+\left[\mathcal{N}\left(0, \sigma_{0}^{2}\right)\right]^{2}
\end{aligned}
$$

By normalising with respect to $\sigma_{0}^{2}$, we observe that of $g_{v_{i}}=$ $\left|r_{v_{i}}\right|^{2} / \sigma_{0}^{2}$ and $g_{u_{j}}=\left|r_{u_{j}}\right|^{2} / \sigma_{0}^{2}$ obey

$$
g_{v_{i}} \sim \chi_{2}^{2}\left(g ; \lambda b_{m_{i}}^{2}\right) ; \quad g_{u_{j}} \sim \chi_{2}^{2}(g)
$$

where $\lambda=\beta / N_{a} \sigma_{0}^{2}$.

Recall from (20) that the correct decision concerning the SD symbols occurs, when $\sum_{i=1}^{N_{a}}\left|r_{v_{i}}\right|^{2}$ attains its maximum. By expressing $N_{a}^{\prime}=\left(N_{r}-N_{a}\right)$, the lower bound of the correct detection probability $\bar{e}_{s, l b}^{p}\left(\lambda, \boldsymbol{b}_{m}\right)$ of the SD symbols may be expressed as in (42), shown at the top of the next page, when the energy pattern based symbols $\boldsymbol{b}_{m}$ were transmitted and the RA pattern $\mathrm{C}(k)$ was activated. To elaborate further, Equation (42-a) serves as the lower bound, since it sets the most strict condition for the correct detection, when each metric $g_{u_{j}}$ of the inactivated RA indices in $\overline{\mathrm{C}}(k)$ is lower than each metric $g_{v_{i}}$ of the activated RA indices in $\mathcal{C}(k)$, while Equation (42-b) follows from the fact that the $N_{a}$ random variables $g_{v_{i}}$ are independent of each other. Furthermore, Equation (42-c) follows from the fact that the $N_{a}^{\prime}$ random variables $g_{u_{j}}$ are independent. After averaging over $\mathcal{C}(k)$, we arrive at expression of (29), since $\mathbb{E}_{\mathcal{C}(k)}\left[\bar{e}_{s, l b}^{p}\left(\lambda, \boldsymbol{b}_{m}\right)\right]=\bar{e}_{s, l b}^{p}\left(\lambda, \boldsymbol{b}_{m}\right)$. As a result, after averaging further over the distribution of $f_{\lambda}(\lambda)$ and taking the expectation over the combinations of $\boldsymbol{b}_{m}$, the analytical SER $e_{s}^{p}$ of the SD symbol may be upper bounded as in (28). In general, $f_{\lambda}(\lambda)$ can be acquired with the aid of the empirical histogram based method. 


$$
\begin{aligned}
\bar{e}_{s, l b}^{p}\left(\lambda, \boldsymbol{b}_{m}\right) & \stackrel{a}{=} \int_{0}^{\infty} P\left(g_{u_{1}}<g_{v_{1}}, \ldots, g_{u_{N_{a}^{\prime}}}<g_{v_{1}}, \ldots, g_{u_{1}}<g_{v_{N_{a}}}, \ldots, g_{u_{N_{a}^{\prime}}}<g_{v_{N_{a}}}\right) P\left(g_{v_{1}}, \ldots, g_{v_{N_{a}}} \mid \lambda, \boldsymbol{b}_{m}\right) d g_{v_{1}} \cdots d g_{v_{N_{a}}} \\
& \stackrel{b}{=} \prod_{i=1}^{N_{a}} \int_{0}^{\infty} P\left(g_{u_{1}}<g_{v_{i}}, \ldots, g_{u_{N_{a}^{\prime}}}<g_{v_{i}}\right) P\left(g_{v_{i}} \mid \lambda, b_{m_{i}}\right) d g_{v_{i}} \\
& \stackrel{c}{=} \prod_{i=1}^{N_{a}} \int_{0}^{\infty} \prod_{u_{j} \in \overline{\mathcal{C}}(k)} P\left(g_{u_{j}}<g_{v_{i}}\right) P\left(g_{v_{i}} \mid \lambda, b_{m_{i}}\right) d g_{v_{i}}=\prod_{i=1}^{N_{a}} \int_{0}^{\infty}\left[F_{\chi_{2}^{2}}(g)\right]^{N_{r}-N_{a}} f_{\chi_{2}^{2}}\left(g ; \lambda b_{m_{i}}^{2}\right) d g
\end{aligned}
$$

\section{APPENDIX D \\ PROOF OF LEMMA II.4}

Since the stage-one detection of (19) significantly affects the stage-two detection of (21), we first introduce the SER $\tilde{e}_{s}^{s}$ of the energy pulse symbols for intensity-based information transfer in the absence of SD symbol errors. By considering (41), for a specific activated RA index $v_{i}=\mathrm{C}(k, i)$, the resultant decision metric is $g_{v_{i}} \sim \chi_{2}^{2}\left(g ; \lambda b_{m_{i}}^{2}\right)$. By dropping the index of $v_{i}$, the average SER becomes

$$
\tilde{e}_{s}^{s} \leq \int_{0}^{\infty} \frac{1}{M} \sum_{m=1}^{M} P_{e}\left(t_{m}, \lambda b_{m}^{2}\right) f_{\lambda}(\lambda) d \lambda=\tilde{e}_{s, u b}^{s}
$$

where for each error event $P_{e}\left(t_{m}, \lambda b_{m}^{2}\right)$, we have

$$
\begin{cases}1-F_{\chi_{2}^{2}}\left(g ; \lambda b_{1}^{2}\right)_{g=t_{1}}, & m=1 \\ F_{\chi_{2}^{2}}\left(g ; \lambda b_{M}^{2}\right)_{g=t_{M-1}}, & m=M \\ F_{\chi_{2}^{2}}\left(g ; \lambda b_{m}^{2}\right)_{g=t_{m-1}}+1-F_{\chi_{2}^{2}}\left(g ; \lambda b_{m}^{2}\right)_{g=t_{m}}, & \text { else }\end{cases}
$$

and the equality in (43) holds, when we set $t_{m}$ as the threshold, satisfying

$$
f_{\chi_{2}^{2}}\left(g ; \lambda b_{m}^{2}\right)_{g=t_{m}}=f_{\chi_{2}^{2}}\left(g ; \lambda b_{m+1}^{2}\right)_{g=t_{m}}
$$

while we may also derive the upper bound by simplifying it as $t_{m}=\lambda\left(b_{m}^{2}+b_{m+1}^{2}\right) / 2$, where $\lambda=\beta / N_{a} \sigma_{0}^{2}$.

Let us now consider the SER in the presence of SD symbol errors. The SER of $e_{s}^{s}$ is constituted by the SER of $\tilde{e}_{s}^{s}$, when the detection of the SD symbol is correct, having a probability of $\left(1-e_{s}^{p}\right)$ plus the SER, when the detection of the SD symbol is erroneous, having a probability of $e_{s}^{p}$, which is jointly expressed as

$$
\begin{aligned}
e_{s}^{s} & \leq\left(1-e_{s}^{p}\right) \tilde{e}_{s, u b}^{s}+e_{s}^{p} \sum_{\ell \neq k} P_{k \mapsto \ell} \underbrace{\frac{N_{c} \tilde{e}_{s, u b}^{s}+N_{d} e_{s}^{o}}{N_{a}}}_{E} \\
& <\left(1-e_{s}^{p}\right) \tilde{e}_{s, u b}^{s}+\frac{e_{s}^{p}}{\left(2^{k_{p}}-1\right)} \sum_{\ell \neq k} \frac{N_{c} \tilde{e}_{s, u b}^{s}+N_{d} e_{s}^{o}}{N_{a}}, \\
& <\left(1-e_{s, u b}^{p}\right) \tilde{e}_{s, u b}^{s}+e_{s, u b}^{p} \frac{\sum_{\ell \neq k} N_{c} \tilde{e}_{s, u b}^{s}+N_{d} e_{s}^{o}}{N_{a}\left(2^{k_{p}}-1\right)} .
\end{aligned}
$$

Regarding the second additive part of (44), the true activated RA pattern $\mathrm{C}(k)$ may be erroneously deemed to be any of the other legitimate RA patterns $\mathcal{C}(\ell) \in \mathcal{C}, \ell \neq k$ with a probability of $P_{k \mapsto \ell}$, which we have to average over. As for the calculation of the per-case error rates $E$, when $\mathcal{C}(k)$ was erroneously detected as a particular $\mathcal{C}(\ell)$, we found that it was constituted by the error rates of $\tilde{e}_{s, u b}^{s}$ for those $N_{c}$ RAs in common (which maybe regarded as being partially correctly detected) and the error rates of $e_{s}^{o}$ for those RAs that were exclusively hosted by $\mathrm{C}(\ell)$, but were excluded from $\mathcal{C}(k)$. Furthermore, since only random noise may be received by those $N_{d}$ RAs in $\mathrm{C}(\ell)$, thus $e_{s}^{o}$ simply represents the SER of the energy pulse symbols as a result of a random guess, i.e. we have $e_{s}^{o}=(M-1) / M$.

Although it is natural that energy patterns with a higher $N_{c}$ would be more likely to cause a erroneous detection, we assume an equal probability of $P_{k \mapsto \ell}=1 /\left(2^{k_{p}}-1\right)$. Since naturally, we have $e_{s}^{o} \geq \tilde{e}_{s, u b}^{s}$, the equal probability assumption leads to the relation of (45). Furthermore, we also have $E \geq \tilde{e}_{s, u b}^{s}$. As a result, (46) holds, since upon replacing $e_{s}^{p}$ by $e_{s, u b}^{p}$ puts more weights on the second additive part, which is more prone to errors.

\section{REFERENCES}

[1] K. Maruyama, F. Nori, and V. Vedral, "The physics of Maxwell's demon and information," Rev. Modern Phys., vol. 81, pp. 1-23, Jan. 2009.

[2] R. Landauer, "Irreversibility and heat generation in the computing process,” IBM J. Res. Develop., vol. 5, no. 3, pp. 183-191, Jul. 1961.

[3] M. Saeedi and I. L. Markov, "Synthesis and optimization of reversible circuits-A survey," ACM Comp. Surveys, vol. 45, no. 2, pp. 1-34, Mar. 2013

[4] S. Toyabe, T. Sagawa, M. Ueda, E. Muneyuki, and M. Sano, "Experimental demonstration of information-to-energy conversion and validation of the generalized Jarzynski equality," Nat. Phys., vol. 6, pp. 988-992, Nov. 2010.

[5] O. L. de Weck, D. Roos, and C. L. Magee, Engineering Systems: Meeting Human Needs in a Complex Technological World. Cambridge, MA, USA: MIT Press, 2011.

[6] L. Varshney, "Transporting information and energy simultaneously," in Proc. IEEE Int. Symp. Inf. Theory, 2008, pp. 1612-1616.

[7] L. Varshney, "On energy/information cross-layer architectures," in Proc. IEEE Int. Symp. Inf. Theory, 2012, pp. 1356-1360.

[8] P. Grover and A. Sahai, "Shannon meets Tesla: Wireless information and power transfer," in Proc. IEEE Int. Symp. Inf. Theory, 2010, pp. 2363 2367.

[9] N. Tesla, "The future of the wireless art," in Proc. Wireless Telegraphy Telephony, 1908, pp. 67-71.

[10] J. Garnica, R. Chinga, and J. Lin, "Wireless power transmission: From far-field to near-field," Proc. IEEE, vol. 101, no. 6, pp. 1321-1331, Jun. 2013.

[11] A. Kurs et al., "Wireless power transfer via strongly coupled magnetic resonances," Science, vol. 317, no. 5834, pp. 83-86, Jul. 2007.

[12] W. C. Brown, "The history of power transmission by radio waves," IEEE Trans. Microw. Theory Techn., vol. 32, no. 9, pp. 1230-1242, Sep. 1984.

[13] W. C. Brown and E. Eves, "Beamed microwave power transmission and its application to space," IEEE Trans. Microw. Theory Techn., vol. 40, no. 6, pp. 1239-1250, Jun. 1992. 
[14] J. McSpadden and J. Mankins, "Space solar power programs and microwave wireless power transmission technology," IEEE Microw. Mag., vol. 3, no. 4, pp. 46-57, Dec. 2002.

[15] J. A. G. Akkermans, M. van Beurden, G. J. N. Doodeman, and H. Visser, "Analytical models for low-power rectenna design," IEEE Antennas Wireless Propag. Lett., vol. 4, pp. 187-190, Jun. 2005.

[16] A. Massa, G. Oliveri, F. Viani, and P. Rocca, "Array designs for longdistance wireless power transmission: State-of-the-art and innovative solutions," Proc. IEEE, vol. 101, no. 6, pp. 1464-1481, Jun. 2013.

[17] A. M. Hawkes, A. R. Katko, and S. A. Cummer, "A microwave metamaterial with integrated power harvesting functionality," Appl. Phys. Lett., vol. 103, no. 16, Oct. 2013, Art. ID. 163901.

[18] A. Osseiran et al., "Scenarios for the 5G mobile and wireless communications: The vision of the METIS project," IEEE Commun. Mag., vol. 52, no. 5, May 2014.

[19] T. Taleb and A. Kunz, "Machine type communications in 3GPP networks: Potential, challenges, solutions," IEEE Commun. Mag., vol. 50, no. 3, pp. 178-184, Mar. 2012.

[20] L. Atzori, A. Iera, and G. Morabito, "The Internet of things: A survey," Comput. Netw., vol. 54, no. 15, pp. 2787-2805, Oct. 2010.

[21] R. Zhang and C. K. Ho, "MIMO broadcasting for simultaneous wireless information and power transfer," IEEE Trans. Wireless Commun., vol. 12, no. 5, pp. 1989-2001, May 2013.

[22] J. Park and B. Clerckx, "Joint wireless information and energy transfer in a two-user MIMO interference channel," IEEE Trans. Wireless Commun., vol. 12, no. 8, pp. 4210-4221, Aug. 2013.

[23] A. Fouladgar and O. Simeone, "On the transfer of information and energy in multi-user systems," IEEE Commun. Lett., vol. 16, no. 11, pp. 1733-1736, Nov. 2012.

[24] A. Fouladgar and O. Simeone, "Information and energy flows in graphical networks with energy transfer and reuse," IEEE Wireless Commun. Lett., vol. 2, no. 4, pp. 371-374, Aug. 2013.

[25] L. Liu, R. Zhang, and K.-C. Chua, "Secrecy wireless information and power transfer with MISO beamforming," IEEE Trans. Signal Process., vol. 62, no. 7, pp. 1850-1863.

[26] L. Liu, R. Zhang, and K.-C. Chua, "Wireless information transfer with opportunistic energy harvesting," IEEE Trans. Wireless Commun., vol. 12, no. 1, pp. 288-300, Jan. 2013.

[27] L. Liu, R. Zhang, and K.-C. Chua, "Wireless information and power transfer: A dynamic power splitting approach," IEEE Trans. Commun., vol. 61, no. 9, pp. 3990-4001, Sep. 2013.

[28] X. Zhou, R. Zhang, and C. K. Ho, "Wireless information and power transfer: Architecture design and rate-energy tradeoff," IEEE Trans. Commun., vol. 61, no. 11, pp. 4757-4767, Nov. 2013.

[29] K. Huang and E. G. Larsson, "Simultaneous information-and-power transfer for broadband downlink systems," IEEE Trans. Signal Process., vol. 61, no. 23, pp. 5972-5986, Dec. 2013.

[30] H. Ju and R. Zhang, "Throughput maximization for wireless powered communication networks," IEEE Trans. Wireless Commun., vol. 13, no. 1, pp. 418-428, Jan. 2014.

[31] V. Chawla and D.-S. Ha, "An overview of passive RFID," IEEE Commun. Mag., vol. 45, no. 9, pp. 11-17, Sep. 2007.

[32] P. Popovski, A. Fouladgar, and O. Simeone, "Interactive joint transfer of energy and information," IEEE Trans. Commun., vol. 61, no. 5, pp. 2086-2097, May 2013.

[33] B. Gurakan, O. Ozel, J. Yang, and S. Ulukus, "Energy cooperation in energy harvesting communications," IEEE Trans. Commun., vol. 61, no. 12, pp. 4884-4898, Dec. 2013.

[34] D. Ng, E. Lo, and R. Schober, "Wireless information and power transfer: Energy efficiency optimization in OFDMA systems," IEEE Trans. Wireless Commun., vol. 12, no. 12, pp. 6352-6370, Dec. 2013.

[35] X. Zhou, R. Zhang, and C. K. Ho, "Wireless information and power transfer in multiuser OFDM systems," IEEE Trans. Wireless Commun., vol. 13, no. 4, pp. 2282-2294, Apr. 2014.

[36] I. Krikidis, S. Timotheou, and S. Sasaki, "RF energy transfer for cooperative networks: Data relaying or energy harvesting?" IEEE Commun. Lett., vol. 16, no. 11, pp. 1772-1775, Nov. 2012.

[37] Z. Xiang and M. Tao, "Robust beamforming for wireless information and power transmission," IEEE Wireless Commun. Lett., vol. 1, no. 4, pp. 372375, Aug. 2012.

[38] X. Chen, C. Yuen, and Z. Zhang, "Wireless energy and information transfer tradeoff for limited feedback multi-antenna systems with energy beamforming," IEEE Trans. Veh. Technol., vol. 63, no. 1, pp. 407-412, Jan. 2014.

[39] X. Zhou, X. Zheng, R. Zhang, and L. Hanzo, "Chip-interleaved optical code division multiple access relying on a photon-counting iterative suc- cessive interference canceller for free-space optical channels," Opt. Exp., vol. 21, no. 13, pp. 15926-15937, Jul. 2013.

[40] L. Li, X. Zhou, R. Zhang, and L. Hanzo, "Performance and capacity analysis of Poisson photon-counting based Iter-PIC OCDMA systems," Optics Exp., vol. 21, no. 22, pp. 25 954-25 967, Nov. 2013.

[41] R. Zhang, L.-L. Yang, and L. Hanzo, "Generalised pre-coding aided spatial modulation," IEEE Trans. Wireless Commun., vol. 12, no. 11, pp. 5434-5443, Nov. 2013.

[42] R. Zhang, L.-L. Yang, and L. Hanzo, "Error probability and capacity analysis of generalised pre-coding aided spatial modulation," IEEE Trans. Wireless Commun., vol. 14, no. 1, pp. 364-375, Jan. 2015. [Online]. Available: http://ieeexplore.ieee.org/stamp/stamp.jsp?arnumber=6877673

[43] C. Peel, B. Hochwald, and A. Swindlehurst, "A vector-perturbation technique for near-capacity multiantenna multiuser communication-Part I: Channel inversion and regularization," IEEE Trans. Commun., vol. 53, no. 1, pp. 195-202, Jan. 2005.

[44] Q. Spencer, A. Swindlehurst, and M. Haardt, "Zero-forcing methods for downlink spatial multiplexing in multiuser MIMO channels," IEEE Trans. Signal Process., vol. 52, no. 2, pp. 461-471, Feb. 2004.

[45] H. Urkowitz, "Energy detection of unknown deterministic signals," Proc. IEEE, vol. 55, no. 4, pp. 523-531, Apr. 1967.

[46] M. D. Renzo, H. Haas, A. Ghrayeb, S. Sugiura, and L. Hanzo, "Spatial modulation for generalized MIMO: Challenges, opportunities and implementation," Proc. IEEE, vol. 102, no. 1, pp. 56-103, Jan. 2014. [Online]. Available: http://eprints.soton.ac.uk/354175/

[47] R. Abu-alhiga and H. Haas, "Subcarrier-index modulation OFDM," in Proc. IEEE Int. Symp. Pers., Indoor Mobile Radio Commun., Sep. 2009, pp. $177-181$.

[48] F. Ellinger et al., "Integrated adjustable phase shifters," IEEE Microw. Mag., vol. 11, no. 6, pp. 97-108, Oct. 2010.

[49] R. Eickhoff, R. Kraemer, I. Santamaria, and L. Gonzalez, "Developing energy-efficient MIMO radios," IEEE Veh. Technol. Mag., vol. 4, no. 1, pp. 34-41, Mar. 2009.

[50] F. Ellinger, U. Jorges, U. Mayer, and R. Eickhoff, "Analysis and compensation of phase variations versus gain in amplifiers verified by $\mathrm{SiGe}$ HBT cascode RFIC," IEEE Trans. Microw. Theory Techn., vol. 57, no. 8, pp. 1885-1894, Aug. 2009.

[51] J. Via, I. Santamaria, V. Elvira, and R. Eickhoff, "A general criterion for analog Tx-Rx beamforming under OFDM transmissions," IEEE Trans. Signal Process., vol. 58, no. 4, pp. 2155-2167, Apr. 2010.

[52] R. Couillet and M. Debbah, Random Matrix Methods for Wireless Communications. New York, NY, USA: Cambridge Univ. Press, 2011.

[53] E. Karipidis, N. Sidiropoulos, and Z.-Q. Luo, "Far-field multicast beamforming for uniform linear antenna arrays," IEEE Trans. Signal Process., vol. 55 , no. 10 , pp. 4916-4927, Oct. 2007.

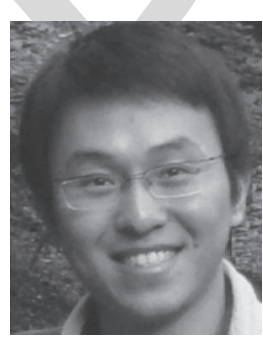

Rong Zhang (S'07-M'09) received the B.Sc. degree from Southeast University, Nanjing, China, in 2003 and the Ph.D. degree from Southampton University, Southampton, U.K., in 2009. Before receiving his Doctorate, he was an Engineer (August 2003-July 2004) at China Telecom and a Research Assistant (January 2006-May 2009) at Mobile Virtual Center of Excellence (MVCE), U.K. After being a Post-Doctoral Researcher (August 2009-July 2012) at Southampton University, he took an industrial consulting leave (August 2012-January 2013) for Huawei Sweden R\&D as a System Algorithms Specialist. Since February 2013, he has been a Lecturer in the CSPC group of ECS, Southampton University. He has $40+$ journals in prestigious publication avenues (e.g., IEEE, OSA) and many more in major conference proceedings. He regularly serves as reviewer for IEEE transactions/journals and has served several times as TPC member/invited session chair of major conferences. He is the recipient of joint funding from MVCE and EPSRC and is also a Visiting Researcher under Worldwide University Network (WUN). More details can be found at http://www.ecs.soton.ac.uk/people/rz. 


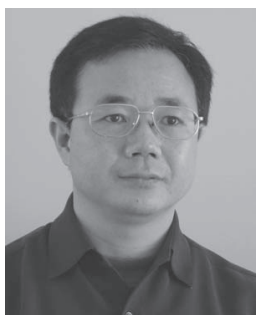

Lie-Liang Yang (M'98-SM'02) received the B.Eng. degree in communications engineering from Shanghai TieDao University, Shanghai, China, in 1988, and the M.Eng. and Ph.D. degrees in communications and electronics from Nothern (Beijing) Jiaotong University, Beijing, China, in 1991 and 1997, respectively. From June 1997 to December 1997, he was a Visiting Scientist with the Institute of Radio Engineering and Electronics, Academy of Sciences of the Czech Republic. Since December 1997, he has been with the University of Southampton, where he is the Professor of wireless communications in the School of Electronics and Computer Science. His research has covered a wide range of topics in wireless communications, networking and signal processing. He has published over 290 research papers in journals and conference proceedings, authored/co-authored three books, and also published several book chapters. The details about his publications can be found at http://www-mobile.ecs.soton.ac.uk/lly/. He is a fellow of the IET, served as an associate editor to the IEEE TRANSACTIONS ON VEHICULAR TECHNOLOGY and Journal of Communications and Networks (JCN), and is currently an associate editor to the IEEE Access and the Security and Communication Networks (SCN) Journal.

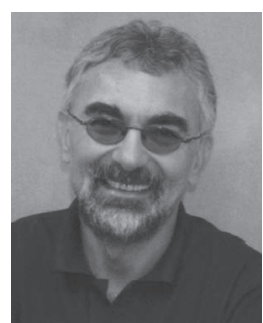

Lajos Hanzo (M'91-SM'92-F'08) received the degree in electronics in 1976 and the Doctorate degree in 1983. In 2009, he was awarded the honorary Doctorate "Doctor Honoris Causa" by the Technical University of Budapest. During his 38-year career in telecommunications he has held various research and academic posts in Hungary, Germany and the U.K. Since 1986, he has been with the School of Electronics and Computer Science, University of Southampton, U.K., where he holds the Chair in telecommunications. He has successfully supervised $80+\mathrm{Ph} . \mathrm{D}$. students, co-authored 20 John Wiley/IEEE Press books on mobile radio communications totalling in excess of 10000 pages, published $1400+$ research entries at IEEE Xplore, acted both as TPC and General Chair of IEEE conferences, presented keynote lectures and has been awarded a number of distinctions. Currently, he is directing a 100-strong academic research team, working on a range of research projects in the field of wireless multimedia communications sponsored by industry, the Engineering and Physical Sciences Research Council (EPSRC) U.K., the European Research Council's Advanced Fellow Grant and the Royal Society's Wolfson Research Merit Award. He is an enthusiastic supporter of industrial and academic liaison and he offers a range of industrial courses. He is also a Governor of the IEEE VTS. During 2008-2012, he was the Editor-in-Chief of the IEEE Press and a Chaired Professor also at Tsinghua University, Beijing. His research is funded by the European Research Council's Senior Research Fellow Grant. For further information on research in progress and associated publications please refer to http://www-mobile.ecs.soton.ac.uk Lajos has $20000+$ citations.

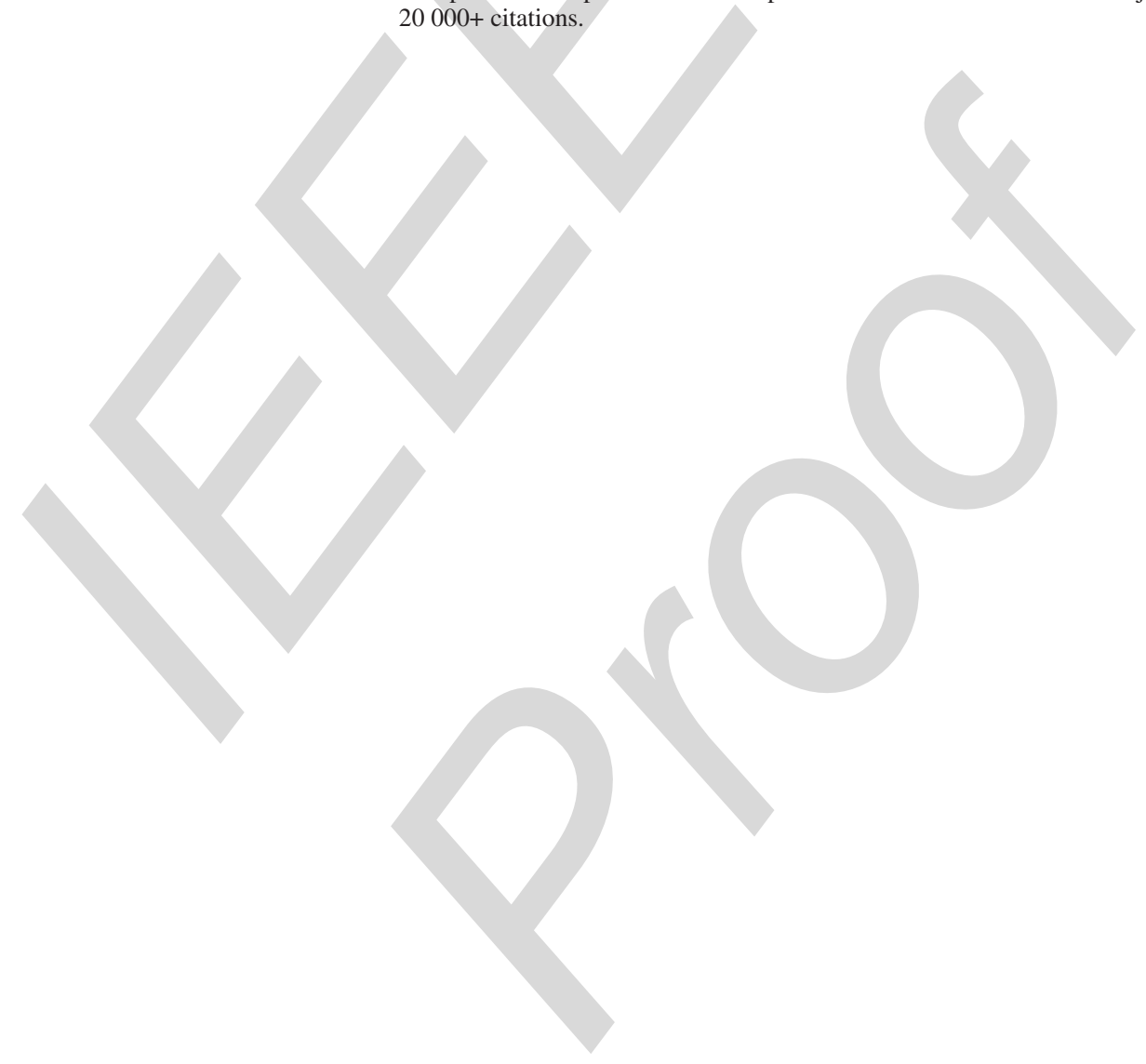

